\title{
Para-Pluriharmonic Maps and Twistor Spaces
}

Dissertation zur Erlangung des Doktorgrades

an der Mathematisch-Naturwissenschaftlichen Fakultät

der Universität Augsburg

vorgelegt von

Matthias Krahe

2007 
Erstgutachter: Prof. Dr. Jost-Hinrich Eschenburg Zweitgutachter: Prof. Dr. Vicente Cortés

Tag der mündlichen Prüfung: 17.7.2007 


\section{Contents}

\begin{tabular}{ll}
\hline Acknowledgements & 5
\end{tabular}

$\begin{array}{ll}\text { Introduction } & 7\end{array}$

$\begin{array}{ll}\text { Chapter 1. Preliminaries } & 11\end{array}$

$\begin{array}{lll}1.1 . & \text { Para-complex vector spaces } & 11\end{array}$

1.2. Para-complex manifolds 13

1.3. Para-hermitian metrics and para-Kähler manifolds 18

\begin{tabular}{lll}
\hline 1.4. & Para-quaternionic Kähler manifolds & 19
\end{tabular}

\begin{tabular}{|lll|}
\hline Chapter 2. & Para-Kähler submanifolds of para-quaternionic & \\
\hline \hline & Kähler manifolds & 23
\end{tabular}

2.1. Para-complex symplectic and contact structures 23

2.2. Para-Kähler submanifolds and Twistor lift 28

\begin{tabular}{lll}
\hline Chapter 3. & Para-pluriharmonic maps into symmetric spaces & 33
\end{tabular}

3.1. Para-pluriharmonic maps 33

$\begin{array}{lll}3.2 . & \text { Isotropic para-pluriharmonic maps } & 40\end{array}$

3.3. Canonical elements and twistor spaces 41

3.4. Isotropic para-pluriharmonic maps into Grassmannians 51

3.5. Para-quaternionic Kähler symmetric spaces 53

\begin{tabular}{lll}
\hline 3.6. & The classical para-quaternionic Kähler symmetric spaces & 56
\end{tabular}

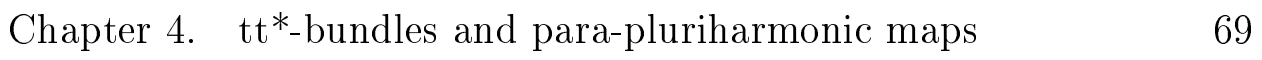

\begin{tabular}{ll}
\hline Bibliography & 73
\end{tabular} 



\section{Acknowledgements}

This work would not have been written but for the support and motivation I received from a number of people. Specifically I would like to thank:

my advisor Prof. Dr. Vicente Cortés for suggesting the topic about para-complex twistor spaces and for the support in Nancy and Hamburg

my advisor Prof. Dr. Jost-Hinrich Eschenburg for suggesting extending this work to pseudo-Riemannian symmetric spaces and for the support in Augsburg,

Marie-Amélie Lawn and Lars Schäfer for their hospitality and discussions about tt*-geometry and associated families,

Christian Boltner, Walter Freyn, Peter Quast and Kerstin Weinl for their friendship and various fruitful and diverting discussions about all kinds of mathematics and non-mathematics,

and above all, my family and my parents. 



\section{Introduction}

Twistor theory goes back to the 1960s, where twistor methods were introduced by R. Penrose to provide an approach to quantum gravity. The general idea is to translate a problem on a differentiable manifold $M$ endowed with a certain geometric structure to a problem on a complex manifold $Z$, the twistor space. Usually, $Z$ is a fibre bundle over $M$, with the fibres over $p \in M$ being complex structures on $T_{p} M$ which are compatible with the geometric structure on $M$. $Z$ carries a canonical almost complex structure, whose integrability can be derived from the properties of $M$.

An important application of twistor theory is the construction of minimal and pluriminimal submanifolds: In 1982, Bryant used the the fibration $\mathbb{C P}^{3} \rightarrow \mathbb{H P}^{1}$ to construct minimal surfaces in $\mathbb{H P}^{1}=\mathrm{S}^{4}[\mathrm{Br}$. This has been generalized to the construction of minimal surfaces in various Grassmannians and symmetric spaces by considering horizontal maps into certain flag manifolds and flag domains [BR, BS]. The reinterpretation of Bryant's work by Lawson $[\mathrm{L}]$ using a birational equivalence $\mathbb{C P}^{3} \sim \mathrm{F}_{1,1,1}\left(\mathbb{C}^{3}\right):=\mathrm{SU}(3) / \mathrm{S}(\mathrm{U}(1) \times \mathrm{U}(1) \times \mathrm{U}(1))$ led to the remarkable observation that the twistor spaces of any two symmetric quaternionic Kähler spaces are birationally equivalent $[\mathrm{Bu}]$.

The purpose of this work is to transfer some of these methods to paracomplex geometry:

A para-complex (or hyperbolic complex) manifold is a smooth manifold with a field of involutions $J_{p}: T_{p} M \rightarrow T_{p} M, J^{2}=1$, such that the \pm 1 -eigendistributions are integrable and of the same dimension. The obvious example is a product of two manifolds $M_{+} \times M_{-}$of the same dimension; in fact, every para-complex manifold is locally of this form. Para-complex and para-quaternionic manifolds have recently been studied in mathematics as well as in physics, where they are related to supersymmetric field theories with Euclidean (rather than Lorentzian) space-times [CMMS1, CMMS2.

Chapter 1: This chapter contains the definitions of para-complex manifolds, para-Kähler manifolds and para-quaternionic Kähler manifolds. We give a proof of the para-complex Dolbeault Lemma, which states that in a para-complex manifold, the $\bar{\partial}$-operator is locally exact (Theorem 1.2.8 and Corollary 1.2.11). 
Chapter 2: Twistor theory of para-quaternionic Kähler manifolds. Extending earlier results by Blair et al. [BDM], it has been shown by Alekseevsky and Cortés [AC2], that for any para-quaternionic Kähler manifold $M$ there is a twistor bundle $\pi: Z \rightarrow M$ of paracomplex structures (actually there is also a twistor bundle $Z^{-} \rightarrow M$ of complex structures, which we neglect here). $Z$ has a canonical paraKähler structure, and the horizontal distribution is a para-holomorphic contact structure. A submanifold $N \subset M$ such that there exists a parallel section of $\left.Z\right|_{N}$ is called para-complex submanifold. If the pseudoRiemannian metric $g_{M}$ is nondegenerate on $T N$, then $N$ is a paraKähler submanifold, and it follows that $N$ is a minimal submanifold (i. e. the trace of the shape operator is zero). Any para-complex submanifold can be lifted to a para-holomorphic horizontal submanifold of $Z$, in other words, para-complex immersions $f: N \rightarrow M$ are precisely the projections of para-holomorphic horizontal immersions $F: N \rightarrow Z$.

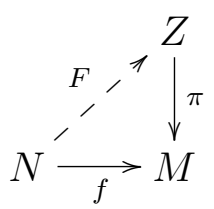

It follows that $N$ has at most half the dimension of $M$, and the paracomplex submanifolds with maximal dimension correspond to the Legendrian submanifolds of $Z$.

Our aim is to show that these Legendrian submanifolds and therefore the maximal para-complex submanifolds of $M$ are locally given by a para-holomorphic function.

The main step will be the proof of the para-complex equivalent of the Darboux theorem, which states that on each para-complex contact manifold, there exist local coordinate charts which identify the contact structure with the standard contact structure on $\mathbf{C}^{2 n+1}$, where $\mathbf{C}$ denotes the algebra of para-complex numbers (Theorem 2.1.11).

Chapter 3: Twistor theory of pseudo-Riemannian symmetric spaces. The notion of twistors can be generalized to the setting where $M$ a pseudo-Riemannian symmetric space (not necessarily paraquaternionic Kähler). There is a twistor construction analogous to the one in chapter 2 , where the role of para-complex immersions is played by isotropic para-pluriharmonic maps (in fact, in the case of paraquaternionic Kähler symmetric spaces, these constructions coincide). A map $f: N \rightarrow M$ is called para-pluriharmonic, if it is harmonic along every para-complex curve in $N$. Under certain conditions, these maps have a one-parameter family $\left(f_{\theta}\right)_{\theta \in \mathbb{R}}$ of deformations, the associated family. A special case occurs when this family is constant in $\theta$; such maps $f$ are called isotropic. We prove the following: Each full isotropic 
para-pluriharmonic map $f: N \rightarrow M$ from a Kähler manifold $N$ into a symmetric space $M=G / G^{\sigma}$ defines a twistor fibration $Z \rightarrow M$ and a holomorphic superhorizontal lift $F: N \rightarrow Z$; vice versa, given such a map $F$, its projection $f=\pi \circ F$ is isotropic para-pluriharmonic (Theorem 3.3.6.

The twistor space is an open subset of the para-complex coset space $\bar{Z}=(G / P) \times(G / P)$, where the para-complex structure is just multiplication by +1 on the first factor and by -1 on the second. If $G$ is a real linear algebraic group, then $P$ is a parabolic subgroup, and thus $G / P$ a real projective variety. This variety has a cell decomposition (Bruhat decomposition) with exactly one cell $N$ in the largest dimension, which is an open (even Zariski-open) and dense subset.

Now, if $Z$ is the twistor space associated to a symmetric para-quaternionic Kähler space, then it turns out that there is a contact isomorphism between the product $N \times N \subset \bar{Z}$ of the big cells and the paracomplex Heisenberg group. It follows that, given the twistor spaces of two symmetric para-quaternionic Kähler spaces of the same dimension, then there is a biholomorphism defined on Zariski-open subsets preserving the contact distribution; moreover, this biholomorphism extends to a birational equivalence. This can be used to transfer the construction of para-pluriharmonic maps from one para-quaternionic Kähler symmetric space to another. Among these spaces is the paracomplex Grassmannian $\mathrm{Gr}_{2}\left(\mathbf{C}^{n+2}\right)$ with corresponding twistor fibration $\mathrm{F}_{1, n, 1}\left(\mathbf{C}^{n+2}\right) \rightarrow \mathrm{Gr}_{2}\left(\mathbf{C}^{n+2}\right)$, where this construction is fairly easy.

Chapter 4: tt $^{*}$-bundles. (Para-)pluriharmonic maps are closely related to a geometric structure in topological field theories called topological-antitopological fusion, or $t t^{*}$-geometry: There is a bijection between $\mathrm{tt}^{*}$-bundles and (para-)pluriharmonic maps into the symmetric space $\mathrm{GL}(p+q) / \mathrm{O}(p, q)$ which admit an associated family (called admissible in [S2]). Here we relate this to the construction of associated families and give a criterion for the (para-)pluriharmonic map to be isotropic.

Differences to the complex theory. For those who are familiar with the twistor theory in the complex case, we point out a few differences to the para-complex case:

- Para-complex manifolds: A para-complex structure defines a splitting of the tangent bundle into eigenbundles - there is no need to consider the para-complexified tangent bundle (at times we do so for convenience, but this yields no new information). 
- Regularity: Para-holomorphic and para-pluriharmonic maps are not necessarily analy tic (any pair of differentiable functions $f_{ \pm}$defines a para-holomorphic map $\left.f_{+} \times f_{-}: \mathbb{R} \times \mathbb{R} \rightarrow \mathbb{R} \times \mathbb{R}\right)$.

- Associated families: All pluriharmonic maps into Riemannian symmetric spaces have associated families, provided that the symmetric space has nonpositive or nonnegative curvature. In the para-complex context, this is different: Unless the symmetric space is flat, there exist para-pluriharmonic maps which do not admit an associated family.

Moreover, the parameter range of the associated family is $\mathbb{R}$ rather than $\mathrm{S}^{1}$. Consequently, in our context the loop group of $G$ will be defined as the set of smooth curves $\mathbb{R} \rightarrow G$.

- Topology: In contrast to hermitian symmetric spaces, parahermitian spaces are not necessarily simply connected, an example being the para-complex projective space

$$
\mathrm{CP}^{n}=\mathrm{SL}(n+1, \mathbb{R}) / \mathrm{S}(\mathrm{GL}(1, \mathbb{R}) \times \mathrm{GL}(n, \mathbb{R})) .
$$

This example also shows that the twistor fibration $G / H \rightarrow$ $G / K$ is not defined globally, if $G_{0}^{\sigma} \subset K \subsetneq G^{\sigma}$ is "too small" (where $H$ is the centralizer of a canonical element and $G^{\sigma}$ denotes the fixed-point set of the Cartan involution). 


\section{CHAPTER 1}

\section{Preliminaries}

\subsection{Para-complex vector spaces}

Definition 1.1.1. Let $V$ be a finite-dimensional real vector space. A para-complex structure on $V$ is an involution $J: V \rightarrow V, J^{2}=\mathrm{id}_{V}$, such that the eigenspaces $V^{ \pm}:=\operatorname{ker}(\mathrm{id} \mp J)$ have the same dimension. The pair $(V, J)$ is then called a para-complex vector space. A homomorphism between para-complex vector spaces $(V, J)$ and $\left(V^{\prime}, J^{\prime}\right)$ is a linear map $\phi: V \rightarrow V^{\prime}$ satisfying $\phi \circ J=J^{\prime} \circ \phi$.

Just like complex vector spaces (i.e. $(V, I)$ with $I^{2}=-1$ ) are vector spaces over the field $\mathbb{C}$, para-complex vector spaces are free modules over the algebra of para-complex numbers:

Definition 1.1.2. The algebra $\mathbf{C}=\mathbb{R} \oplus j \mathbb{R}$ of para-complex numbers (also called split complex or hyperbolic complex numbers) is the real algebra generated by 1 and $j$, with the relation $j^{2}=1$ (in fact, $\mathbf{C}$ is the Clifford algebra $\mathrm{Cl}(0,1))$.

The conjugation map ${ }^{-}: \mathbf{C} \rightarrow \mathbf{C}$ is defined by $\overline{x+j y}=x-j y$ for $x, y \in$ $\mathbb{R}$. The real numbers $x=\operatorname{Re}(z):=(z+\bar{z}) / 2$ and $y=\operatorname{Im}(z):=(z-\bar{z}) / 2 j$ are called the real and imaginary part of $z=x+j y$, respectively. Note that $\bar{z} z=x^{2}-y^{2} \in \mathbb{R}$. Thus, the group of units is $\mathbf{C}^{*}=\{x+j y: x \neq$ $\pm y\}=\{z \in \mathbf{C}: z \notin(1 \pm j) \mathbb{R}\}$. It has four connected components; in fact, each $z \in \mathbf{C}^{*}$ can be written uniquely as $\pm r e^{j \theta}$ or $\pm j r e^{j \theta}$, where $r \in \mathbb{R}^{+}, \theta \in \mathbb{R}$, and $e^{j \theta}=\cosh (\theta)+j \sinh (\theta)$. This is the para-complex analogue of the polar decomposition; the unit circle is replaced by the four hyperbolas $\left\{(x, y) \in \mathbb{R}^{2}: x^{2}-y^{2}= \pm 1\right\}$.

The free module $\mathbf{C}^{n}$ is a para-complex vector space, with the multiplication by $j$ as para-complex structure. Conversely, any para-complex vector space $(V, J)$ can be regarded as a $\mathbf{C}$-module via $(x+j y) v:=x v+$ $y J v$. In order to see that this module is free, let $\left\{v_{1}^{+}, \ldots, v_{n}^{+}, v_{1}^{-}, \ldots, v_{n}^{-}\right\}$ be a basis of $V$, such that $v_{a}^{ \pm} \in V^{ \pm}$. Then the equation $\sum\left(x^{a}+j y^{a}\right)\left(v_{a}^{+}+\right.$ $\left.v_{a}^{-}\right)=\frac{1+j}{2} \sum\left(x^{a}+y^{a}\right) v_{a}^{+}+\frac{1-j}{2} \sum\left(x^{a}-y^{a}\right) v_{a}^{-}$for coeffiecients $x^{a}+j y^{a} \in \mathbf{C}$ shows that $\left\{w_{1}, \ldots, w_{n}\right\}, w_{a}:=v_{a}^{+}+v_{a}^{-}$is a free basis of the $\mathbf{C}$-module $V$. Homomorphisms between para-complex vector spaces correspond to C-linear maps. 
REMARK 1.1.3. Since $\mathbf{C}$ is not a field, some basic facts from linear algebra must be treated with caution:

(1) Let $\phi: V \rightarrow V^{\prime}$ be $\mathbf{C}$-linear. Then the image and the kernel of $\phi$ are $J$-invariant real subspaces of $V^{\prime}$ and $V$, respectively. They are not necessarily para-complex subspaces, because the dimensions of $\phi\left(V^{ \pm}\right)$, resp. $\operatorname{ker}(\phi)^{ \pm}$may not be the same. Of course, it still holds that

$$
\phi \text { injective } \Longleftrightarrow \operatorname{ker}(\phi)=\{0\} .
$$

(2) Let $\phi: V \rightarrow V$ be a $\mathbf{C}$-linear endomorphism and let $z=x+j y \in$ C. Then the eigenspace $V_{z}:=\operatorname{ker}(\phi-z \cdot \mathrm{id})$ is $J$-invariant, and therefore we have $V_{z}=V_{z}^{+} \oplus V_{z}^{-}$. The $V_{z}^{ \pm}$are eigenspaces of $\phi$ with real eigenvalue $(x \pm y)$. In particular, eigenspaces to different para-complex eigenvalues need not be disjoint.

(3) The C-span of a single vector $v \in V$ is not necessarily a paracomplex vector space; this motivates the following definition:

Definition 1.1.4. Let $(V, J)$ be a para-complex vector space. A vector $v \in V$ is called regular, if it fulfills the following equivalent conditions:

(1) $v \notin V^{+}$and $v \notin V^{-}$.

(2) $\mathbf{C} \cdot v$ is a one-dimensional (over $\mathbf{C}$ ) para-complex vector space.

(3) $v$ and $J v$ are linearly independent over $\mathbb{R}$.

(4) For $z \in \mathbf{C}$ it holds that $(z v=0 \Longleftrightarrow z=0)$.

Proof of The equivalence. (1) $\Leftrightarrow(2)$ : We write $v=v^{+}+v^{-} \in$ $V^{+} \oplus V^{-}$. Then $(\mathbf{C} \cdot v)^{ \pm}=\mathbb{R} \cdot v^{ \pm}$. So $\mathbf{C} \cdot v$ is a one-dimensional paracomplex vector space, if and only if $v^{-} \neq 0$ and $v^{+} \neq 0$. (1) $\Rightarrow(3)$ : We may assume that $v \neq 0$. If $J v=r v, r \in \mathbb{R}$, then $v=J^{2} v=r^{2} v$, so $r= \pm 1$. (3) $\Rightarrow(1)$ is trivial. (3) $\Leftrightarrow$ (4) follows immediately from $(x+j y) v=x v+y J v$.

The following example is fundamental since it illustrates the principle that para-complex objects can be described either by using para-complex numbers or by using real numbers:

EXAMPLE 1.1.5. The map

$$
\begin{aligned}
\left(\mathbf{C}^{n}, j\right) & \cong\left(\mathbb{R}^{n} \times \mathbb{R}^{n},(\mathrm{id}) \times(-\mathrm{id})\right) \\
\left(x^{a}+j y^{a}\right)_{a=1, \ldots, n} & \mapsto\left(x^{a}+y^{a}, x^{a}-y^{a}\right)_{a=1, \ldots, n} \\
\left(\frac{1+j}{2} z_{+}^{a}+\frac{1-j}{2} z_{-}^{a}\right)_{a=1, \ldots, n} & \longleftrightarrow\left(z_{+}^{a}, z_{-}^{a}\right)_{a=1, \ldots, n}
\end{aligned}
$$


is an isomorphism of para-complex vector spaces. It induces an isomorphism of groups

$$
\begin{aligned}
\mathrm{GL}(n, \mathbf{C}) & \cong \mathrm{GL}(n, \mathbb{R}) \times \mathrm{GL}(n, \mathbb{R}) \\
B+j C & \mapsto\left(\begin{array}{cc}
B+C & 0 \\
0 & B-C
\end{array}\right) \\
\frac{1+j}{2} A_{+}+\frac{1-j}{2} A_{-} & \longleftrightarrow\left(\begin{array}{cc}
A_{+} & 0 \\
0 & A_{-}
\end{array}\right)
\end{aligned}
$$

Lemma 1.1.6. Let $A_{+}, A_{-} \in \mathrm{GL}(n, \mathbb{R})$. Then

$$
\operatorname{det}_{\mathbf{C}}\left(\frac{1+j}{2} A_{+}+\frac{1-j}{2} A_{-}\right)=\frac{1+j}{2} \operatorname{det}\left(A_{+}\right)+\frac{1-j}{2} \operatorname{det}\left(A_{-}\right) .
$$

ProOF. This follows immediately from the Leibniz formula and from $\left(\frac{1+j}{2}\right)^{2}=\frac{1+j}{2},\left(\frac{1-j}{2}\right)^{2}=\frac{1-j}{2}, \frac{1+j}{2} \frac{1-j}{2}=0$.

COROLlary 1.1.7.

$\operatorname{det}_{\mathbf{C}}\left(\frac{1+j}{2} A_{+}+\frac{1-j}{2} A_{-}\right) \in \mathbf{C}^{*} \Longleftrightarrow \operatorname{det}\left(A_{+}\right) \neq 0 \wedge \operatorname{det}\left(A_{-}\right) \neq 0$.

Corollary 1.1.8.

$$
\mathrm{SL}(n, \mathbf{C}) \cong \mathrm{SL}(n, \mathbb{R}) \times \mathrm{SL}(n, \mathbb{R})
$$

\subsection{Para-complex manifolds}

DEFINITION 1.2.1. An almost para-complex structure on a manifold $M$ is a smooth endomorphism field $J$ on $T M$, such that for every $p \in M, J_{p}$ is a para-complex structure on $T_{p} M$. An almost para-complex structure is said to be integrable, if the eigendistributions $T^{ \pm} M:=\operatorname{ker}(\mathrm{id} \mp J)$ are integrable. In this case, $(M, J)$ is called a para-complex manifold.

As a consequence of the Frobenius theorem - see for example CMMS1, Chapter 2.1] - we have

Proposition 1.2.2. Let $(M, J)$ be an almost para-complex manifold. Then the following conditions are equivalent:

(1) $J$ is integrable

(2) $\left[T^{+} M, T^{+} M\right] \subset T^{+} M$ and $\left[T^{-} M, T^{-} M\right] \subset T^{-} M$

(3) $N_{J}=0$, where $N_{J}$ is the Nijenhuis-Tensor

$$
N_{J}(X, Y):=[X, Y]+[J X, J Y]-J[X, J Y]-J[J X, Y]
$$

(4) For any point $p \in M$, there exist local real coordinates $\left(z_{+}^{1}, \ldots, z_{+}^{n}, z_{-}^{1}, \ldots, z_{-}^{n}\right): U \stackrel{\sim}{\rightarrow} \tilde{U}_{+} \times \tilde{U}_{-} \subset \mathbb{R}^{n} \times \mathbb{R}^{n}$ defined on an open neighbourhood $U$ of $p$, such that $d z_{ \pm}^{a} \circ J= \pm d z_{ \pm}^{a}$. 
(4') For any point $p \in M$, there exist local para-complex coordinates $\left(z^{1}, \ldots, z^{n}\right): U \stackrel{\sim}{\rightarrow} \tilde{U} \subset \mathbf{C}^{n}$ defined on an open neighbourhood $U$ of $p$, such that $d z^{a} \circ J=j d z^{a}$.

These coordinates are para-holomorphic in the following sense:

Definition 1.2.3. A smooth map $\phi:\left(M, J_{M}\right) \rightarrow\left(M^{\prime}, J_{M^{\prime}}\right)$ between para-complex manifolds is called para-holomorphic (or just holomorphic, if no confusion is possible), if

$$
d \phi \circ J_{M}=J_{M^{\prime}} \circ d \phi .
$$

Para-holomorphic maps $(M, J) \rightarrow(\mathbf{C}, j)$ or $(M, J) \rightarrow(\mathbb{R} \times \mathbb{R},($ id $) \times$ $(-\mathrm{id}))$ are called para-holomorphic functions.

DEFINITION 1.2.4. Let $M$ be a para-complex manifold and let $z^{a}=$ $\frac{1+j}{2} z_{+}^{a}+\frac{1-j}{2} z_{-}^{a}, a=1 \ldots n$, be a system of local holomorphic coordinates. Then $\left(d z^{a}, d \bar{z}^{a}\right)_{a=1 \ldots n}$ is local frame of the complexified cotangent bundle $T^{*} M \otimes \mathbf{C}$. The dual frame of $T M \otimes \mathbf{C}$ is given by

$$
\begin{aligned}
\frac{\partial}{\partial z^{a}} & :=\frac{1+j}{2} \frac{\partial}{\partial z_{+}^{a}}+\frac{1-j}{2} \frac{\partial}{\partial z_{-}^{a}} \\
\frac{\partial}{\partial \bar{z}^{a}} & :=\frac{1-j}{2} \frac{\partial}{\partial z_{+}^{a}}+\frac{1+j}{2} \frac{\partial}{\partial z_{-}^{a}} .
\end{aligned}
$$

Proposition 1.2.5. Let $M$ be a para-complex manifold and let $z^{a}=$ $\frac{1+j}{2} z_{+}^{a}+\frac{1-j}{2} z_{-}^{a}, a=1 \ldots n$, be a system of local holomorphic coordinates. Then a smooth function $f: M \rightarrow \mathbf{C}$ is para-holomorphic, if and only if it fulfills the Cauchy-Riemann equations

$$
\frac{\partial f}{\partial \bar{z}^{a}}=0
$$

ProOF. We write $f=\frac{1+j}{2} f_{+}+\frac{1-j}{2} f_{-}$, where $f_{ \pm}:=\operatorname{Re}(f) \pm \operatorname{Im}(f)$. Then it is easy to see that the Cauchy-Riemann equations are equivalent to

$$
\frac{\partial f_{+}}{\partial z_{-}^{a}}=\frac{\partial f_{-}}{\partial z_{+}^{a}}=0
$$

But this in turn is just a rewriting of $d f \circ J_{M}=J_{\mathbb{R} \times \mathbb{R}} \circ d f$.

LEMMA 1.2.6. Let $U \subset \mathbf{C}$ be a simply connected domain and let $f: U \rightarrow$ $\mathrm{C}$ be a para-holomorphic function. Then $f$ has an antiderivative, i.e. a para-holomorphic function $F$, such that $\frac{\partial F}{\partial z}=f$.

Proof. Since $d(f d z)=\frac{\partial f}{\partial \bar{z}} d \bar{z} \wedge d z=0$, the C-valued 1-form $f d z$ is closed, hence exact, i. e. $f d z=d F=\frac{\partial F}{\partial z} d z+\frac{\partial F}{\partial \bar{z}} d \bar{z}$. It follows that $\frac{\partial F}{\partial z}=f$ and $\frac{\partial F}{\partial \bar{z}}=0$. 


\subsubsection{Bigrading of differential forms, Dolbeault sequences.}

Real version. Let $(M, J)$ be an almost para-complex manifold. The decomposition $T^{*} M=\left(T^{*} M\right)^{+} \oplus\left(T^{*} M\right)^{-}$into the \pm 1 -eigenspaces of $J^{*}$ induces a bigrading on exterior and differential forms:

$$
\begin{aligned}
& \wedge^{k} T^{*} M=\bigoplus_{p+q=k} \wedge^{(+p,-q)} T^{*} M \\
& \Omega^{k}(M)=\bigoplus_{p+q=k} \Omega^{(+p,-q)}(M)
\end{aligned}
$$

DEFinition 1.2.7. The elements of $\Omega^{(+p,-q)}(M)$ are called differential forms of degree $(+p,-q)$.

If $J$ is integrable, then we can use holomorphic coordinates to show that $d \Omega^{(+p,-q)}(M) \subset \Omega^{(+(p+1),-q)}(M) \oplus \Omega^{(+p,-(q+1))}(M)$. Hence the exterior derivative splits as $d=\partial_{+}+\partial_{-}$, where

$$
\begin{aligned}
& \partial_{+}: \Omega^{(+p,-q)}(M) \rightarrow \Omega^{(+(p+1),-q)}(M) \\
& \partial_{-}: \Omega^{(+p,-q)}(M) \rightarrow \Omega^{(+p,-(q+1))}(M)
\end{aligned}
$$

From $d^{2}=0$ we get $\partial_{+}^{2}=\partial_{-}^{2}=\partial_{+} \partial_{-}+\partial_{-} \partial_{+}=0$, so for any paracomplex manifold the operators $\partial_{ \pm}$can be used to define real versions of the Dolbeault sequence. This sequence is locally exact:

THEOREM 1.2.8. Let $U=U_{+} \times U_{-}$be a para-complex manifold (with the para-complex structure on $T U_{+} \times T U_{-}$given by $\left.\mathrm{id} \times-\mathrm{id}\right)$, such that both $U_{+}$and $U_{-}$are contractible.

Then any $\partial_{+}$-closed form $\omega \in \Omega^{(+p,-q)}(U), p \geq 1$, is $\partial_{+}$-exact. Likewise, any $\partial_{-}$-closed form $\omega \in \Omega^{(+p,-q)}(U), q \geq 1$, is $\partial_{-}$-exact.

REMARK.

(1) From Proposition 1.2.2 (4) it follows that each point in an arbitrary para-complex manifold has a neighbourhood $U$ of this form.

(2) The condition that $U$ be contractible is not sufficient, as shown by the following conterexample: Let

$$
U:=\left(\mathbb{R}^{2} \times \mathbb{R}^{2}\right) \backslash\left(\{0\} \times\{0\} \times \mathbb{R}_{\leq 0} \times \mathbb{R}\right)
$$

and $\omega\left(z_{+}, w_{+}, z_{-}, w_{-}\right):=f\left(z_{-}\right) \frac{z_{+} d w_{+}-w_{+} d z_{+}}{z_{+}^{2}+w_{+}^{2}}$, where $f\left(z_{-}\right)$is a smooth nonzero function that vanishes on $\mathbb{R}_{>0}$. Then $\omega$ is a smooth $(1,0)$-form, which is $\partial_{+}$-closed, but not $\partial_{+}$-exact.

Proof of The TheOREM. For the proof of the first statement, let $\omega \in \Omega^{(+p,-q)}(U), p \geq 1$, and $\partial_{+} \omega=0$. Let the contraction maps on $U_{+}$ and $U_{-}$be denoted by $h_{+}$and $h_{-}$respectively. We can define a paraholomorphic homotopy map $h:[0,1]^{2} \times U \rightarrow U$ by $h_{\left(s_{+}, s_{-}\right)}\left(u_{+}, u_{-}\right)=$ 
$\left(h_{s_{+}}\left(u_{+}\right), h_{s_{-}}\left(u_{-}\right)\right)$. Then $h_{(1,1)}=\mathrm{id}_{U}$ and $d h_{(0,1)} \cdot T^{+} U=0$ and therefore, since $p \geq 1$,

$$
\begin{aligned}
\omega-0 & =h_{(1,1)}^{*} \omega-h_{(0,1)}^{*} \omega \\
& =\int_{0}^{1} \frac{d}{d s_{+}}\left(h_{\left(s_{+}, 1\right)}^{*} \omega\right) d s_{+}
\end{aligned}
$$

In order to compute the integrand, we write $h_{\left(s_{+}, 1\right)}=h \circ \Psi_{s_{+}} \circ \imath$, where $\imath$ is the embedding $U \rightarrow\{0\} \times\{1\} \times U \subset[0,1]^{2} \times U$ and $\Psi$ is the flow of the vector field $\frac{\partial}{\partial s_{+}}$on $[0,1]^{2} \times U$. Then by Cartan's formula we have

$$
\begin{aligned}
\frac{d}{d s_{+}} h_{\left(s_{+}, 1\right)}^{*} \omega= & \imath^{*} \Psi_{s_{+}}^{*}\left(\mathcal{L}_{s_{+}}\left(h^{*} \omega\right)\right) \\
= & \left.\left.\imath^{*} \Psi_{s_{+}}^{*}\left(\frac{\partial}{\partial s_{+}}\right\lrcorner d\left(h^{*} \omega\right)+d\left(\frac{\partial}{\partial s_{+}}\right\lrcorner h^{*} \omega\right)\right) \\
= & \left.\imath^{*} \Psi_{s_{+}}^{*}\left(\frac{\partial}{\partial s_{+}}\right\lrcorner \partial_{+}\left(h^{*} \omega\right)+\partial_{+}\left(\frac{\partial}{\partial s_{+}}\right\lrcorner h^{*} \omega\right) \\
& \left.\left.\left.\quad+\frac{\partial}{\partial s_{+}}\right\lrcorner \partial_{-}\left(h^{*} \omega\right)+\partial_{-}\left(\frac{\partial}{\partial s_{+}}\right\lrcorner h^{*} \omega\right)\right)
\end{aligned}
$$

Because the maps $\imath, \Psi_{s_{+}}, h$ are para-holomorphic, the respective pullback maps commute with $\partial_{ \pm}$and preserve the types of differential forms. Hence the sum of the terms containing $\partial_{-}$must be zero, since these terms are of degree $(+(p-1),-(q+1))$ and the rest is of degree $(+p,-q)$. Further, by assumption we have $\partial_{+}\left(h^{*} \omega\right)=h^{*} \partial_{+} \omega=0$ and thus the expression reduces to

$$
\left.\frac{d}{d s_{+}} h_{\left(s_{+}, 1\right)}^{*} \omega=\partial_{+}\left(\imath^{*} \Psi_{s_{+}}^{*}\left(\frac{\partial}{\partial s_{+}}\right\lrcorner h^{*} \omega\right)\right) .
$$

Inserting this in (1) yields

$$
\left.\omega=\partial_{+}\left(\int_{0}^{1} \imath^{*} \Psi_{s_{+}}^{*}\left(\frac{\partial}{\partial s_{+}}\right\lrcorner h^{*} \omega\right) d s_{+}\right)
$$

The proof of the second statement is completely analogous.

Para-complex version. There is also a para-complex version of the Dolbeault sequence defined on the para-complexified cotangent bundle $T^{*} M^{\mathbf{c}}:=T^{*} M \otimes_{\mathbb{R}} \mathbf{C}:$ The $\mathbf{C}$-linear extension of $J^{*}: T^{*} M \rightarrow$ $T^{*} M$ to an endomorphism field on $T^{*} M^{\mathbf{c}}$ yields a decomposition $T^{*} M^{\mathbf{c}}=$ $\wedge^{(1,0)} T^{*} M \oplus \wedge^{(0,1)} T^{*} M$ into the $\pm j$-eigenbundles

$$
\begin{aligned}
& \wedge^{(1,0)} T^{*} M=\left\{\alpha+j J \alpha: \alpha \in T^{*} M\right\} \\
& \wedge^{(0,1)} T^{*} M=\left\{\alpha-j J \alpha: \alpha \in T^{*} M\right\}
\end{aligned}
$$


This decomposition extends to a bigrading of $\mathbf{C}$-valued exterior and differential forms:

$$
\begin{aligned}
& \wedge^{k} T^{*} M^{\mathbf{c}}=\bigoplus_{p+q=k} \wedge^{(p, q)} T^{*} M \\
& \Omega^{k}(M)^{\mathbf{c}}=\bigoplus_{p+q=k} \Omega^{(p, q)}(M)
\end{aligned}
$$

If $J$ is integrable, then the $\mathbf{C}$-linear extension of the exterior derivative splits as $d^{\mathbf{c}}=\partial+\bar{\partial}$, where

$$
\begin{aligned}
& \partial: \Omega^{(p, q)}(M) \rightarrow \Omega^{(p+1, q)}(M) \\
& \bar{\partial}: \Omega^{(p, q)}(M) \rightarrow \Omega^{(p, q+1)}(M)
\end{aligned}
$$

Definition 1.2.9. The elements of $\Omega^{(p, q)}(M)$ are called para-complex differential forms of degree $(p, q)$. A $\bar{\partial}$-closed $(p, 0)$-form is called paraholomorphic.

From $d^{2}=0$ we get $\partial^{2}=\bar{\partial}^{2}=\partial \bar{\partial}+\bar{\partial} \partial=0$, so for any para-complex manifold the operators $\bar{\partial}, \partial$ can be used to define para-complex versions of the Dolbeault sequence. The real and para-complex versions are related in the following way:

Proposition 1.2.10. For a para-complex manifold $M$, there is an $(\mathbb{R}$ linear) isomorphism

$$
\begin{aligned}
\wedge^{(+p,-q)} T^{*} M \times \wedge^{(+q,-p)} T^{*} M & \stackrel{\varphi}{\rightarrow} \wedge^{(p, q)} T^{*} M \\
\left(\eta, \eta^{\prime}\right) & \mapsto \frac{1+j}{2} \eta+\frac{1-j}{2} \eta^{\prime}
\end{aligned}
$$

such that the following diagram commutes:

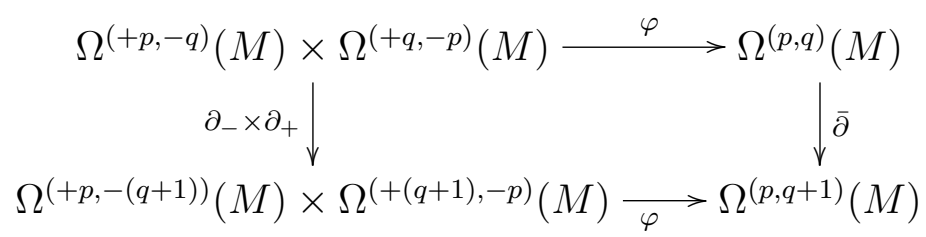

ProOF. The fact that the image of $\varphi$ is contained in $\wedge^{(p, q)} T^{*} M$ is an easy consequence of $\left(\frac{1 \pm j}{2}\right)^{2}=\frac{1 \pm j}{2}, \frac{1+j}{2} \frac{1-j}{2}=0$ and of the equations

$$
\begin{aligned}
\frac{1+j}{2} d z_{+}^{a}=\frac{1+j}{2} d z^{a} & \frac{1-j}{2} d z_{-}^{a}=\frac{1-j}{2} d z^{a} \\
\frac{1+j}{2} d z_{-}^{a}=\frac{1+j}{2} d \bar{z}^{a} & \frac{1-j}{2} d z_{+}^{a}=\frac{1-j}{2} d \bar{z}^{a}
\end{aligned}
$$

for local holomorphic coordinates $\left(z_{ \pm}^{a}\right)_{k=1 \ldots n}$ and $z^{a}=\frac{1+j}{2} z_{+}^{a}+\frac{1-j}{2} z_{-}^{a}$. The map $\varphi$ is clearly a bijection, with the inverse given by

$$
\omega \mapsto(\operatorname{Re}(\omega)+\operatorname{Im}(\omega), \operatorname{Re}(\omega)-\operatorname{Im}(\omega)) .
$$

For the commutativity of the diagram, it is sufficient to consider functions, i. e. the case $f, f^{\prime} \in \Omega^{(+0,-0)}$. Then we calculate $\bar{\partial}\left(\frac{1+j}{2} f\right)=$ 
$\left(\frac{1-j J^{*}}{2} d\right)\left(\frac{1+j}{2} f\right)=\frac{1+j}{2}\left(\frac{1-J^{*}}{2} d f\right)=\frac{1+j}{2} \partial_{-} f$ and in the same way $\bar{\partial} \frac{1-j}{2} f^{\prime}=$ $\frac{1-j}{2} \partial_{+} f^{\prime}$.

Corollary 1.2.11. For $U=U_{+} \times U_{-}$with $U_{+}$and $U_{-}$contractible, any $\bar{\partial}$-closed form $\omega \in \Omega^{(p, q)}(U), q \geq 1$, is $\bar{\partial}$-exact.

\subsection{Para-hermitian metrics and para-Kähler manifolds}

We recall that a pseudo-Euclidean vector space is a real vector space $V$ equipped with a nondegenerate symmetric bilinear form $g: V \times V \rightarrow \mathbb{R}$.

If $W \subset V$ is a subspace with orthogonal complement $W^{\perp}:=\{v \in V$ : $g(w, v)=0 \forall w \in W\}$, then $W$ is called

- nondegenerate, if $W^{\perp} \cap W=\{0\}$

- degenerate, if $W^{\perp} \cap W \neq\{0\}$

- totally degenerate, if $W^{\perp} \supset W$, i. e. if $\left.g\right|_{W \times W}=0$.

FACT 1.3.1. A subspace $W$ is totally degenerate if and only if it consists only of degenerate vectors.

ProOF. This follows from the polarization identity $4 g\left(w, w^{\prime}\right)=$ $g\left(w+w^{\prime}, w+w^{\prime}\right)-g\left(w-w^{\prime}, w-w^{\prime}\right)$.

Definition 1.3.2. Let $(V, J)$ be a para-complex vector space. A pseudoEuclidean scalar product $g$ on $V$ is said to be compatible with $J$ or para-hermitian, if $J$ is $g$-skew, i. e. if $g(J \cdot, \cdot)+g(\cdot, J \cdot)=0$.

Since $J^{2}=1$, this is equivalent to $J$ being an anti-isometry, i.e. $g(J \cdot, J \cdot)=-g$. In particular, $g$ has real signature $(n, n)$ (where $n=$ $\left.\frac{1}{2} \operatorname{dim}_{\mathbb{R}} V\right)$, and the subspaces $V^{ \pm}$are totally degenerate.

On $V=\mathbf{C}^{n}$ the standard para-hermitian scalar product is given by

$$
g\left(\left(z^{1}, \ldots, z^{n}\right),\left(w^{1}, \ldots, w^{n}\right)\right)=\operatorname{Re}\left(\sum_{a} \overline{z^{a}} w^{a}\right) .
$$

The group of $\mathbf{C}$-linear automorphisms of $\mathbf{C}^{n}$ preserving $g$ is called the para-unitary group

$$
\mathrm{U}^{\pi}(n):=\left\{A \in \operatorname{GL}(n, \mathbf{C}): A^{*} A=\mathbf{1}_{n}\right\} \quad\left(\text { where } A^{*}:=\bar{A}^{t}\right) .
$$

It is easy to see that

$$
\mathrm{U}^{\pi}(n)=\left\{\frac{1+j}{2} B+\frac{1-j}{2} B^{-t}: B \in \mathrm{GL}(n, \mathbb{R})\right\}\left(\text { where } B^{-t}:=\left(B^{-1}\right)^{t}\right) .
$$

Further by Lemma 1.1 .6 we have $\operatorname{det}_{\mathbf{C}}\left(\frac{1+j}{2} B+\frac{1-j}{2} B^{-t}\right)=\frac{1+j}{2} \operatorname{det}(B)+$ $\frac{1-j}{2} \operatorname{det}(B)^{-1} ;$ this proves

$$
\begin{aligned}
\mathrm{U}^{\pi}(n) & \cong \mathrm{GL}(n, \mathbb{R}) \\
\operatorname{SU}^{\pi}(n) & \cong \operatorname{SL}(n, \mathbb{R})
\end{aligned}
$$


Under the identification $\mathbf{C}^{n} \cong \mathbb{R}^{n} \times \mathbb{R}^{n}$, the standard para-hermitian scalar product corresponds to the symmetric bilinear form given by the matrix

$$
E:=\left(\begin{array}{cc}
\mathbf{0}_{n} & \mathbf{1}_{n} \\
\mathbf{1}_{n} & \mathbf{0}_{n}
\end{array}\right)
$$

The isometry group is $\left\{X \in \mathrm{GL}(2 n, \mathbb{R}): X^{t} E X=E\right\} \cong \mathrm{O}(n, n)$, and $\mathrm{U}^{\pi}(n)$ is identified with the subgroup

$$
\left\{\left(\begin{array}{cc}
B & \mathbf{0}_{n} \\
\mathbf{0}_{n} & B^{-t}
\end{array}\right): B \in \mathrm{GL}(n, \mathbb{R})\right\} .
$$

Proposition 1.3.3. Every para-hermitian vector space has a unitary basis, i.e. a free C-basis $\left(e_{a}\right)_{a=1 \ldots n}$ in which the metric is given by $g\left(e_{a}, e_{b}\right)=\delta_{a b}$.

Proof. Let $V$ be a para-hermitian vector space, $\operatorname{dim}_{\mathbf{C}}(V)=n . V$ is nondegenerate with respect to $g$, so there is a vector $e_{1}$, such that $g\left(e_{1}, e_{1}\right) \neq 0$. After multiplying $e_{1}$ by $\sqrt{g\left(e_{1}, e_{1}\right)}$ (if $g\left(e_{1}, e_{1}\right)>0$ ) or by $j \sqrt{-g\left(e_{1}, e_{1}\right)}$ (if $g\left(e_{1}, e_{1}\right)<0$ ), we may assume that $g\left(e_{1}, e_{1}\right)=1$. It follows that $e_{1} \notin V^{ \pm}$, so $e_{1}$ is regular and $\mathbf{C} \cdot e_{1}$ is a 1 -dimensional para-complex subspace. The orthogonal complement $\left(\mathbf{C} \cdot e_{1}\right)^{\perp}$ is an $(n-1)$-dimensional para-complex subspace, which is nondegenerate, so we can proceed by induction.

DEFINITION 1.3.4. A para-Kähler manifold is an almost para-complex manifold $(M, J)$ equipped with a compatible pseudo-Riemannian metric $g$, such that $D J=0$, where $D$ denotes the Levi-Civita connection.

Equivalently, a para-Kähler manifold is a pseudo-Riemannian manifold with holonomy group $\mathrm{GL}(n, \mathbb{R}) \subset \mathrm{O}(n, n)$.

From the parallelity of $J$ it follows that $N_{J}=0$ (in particular, $(M, J)$ is a para-complex manifold) and that the $(+1,-1)$-form $\omega:=g(\cdot, J \cdot)$ is also parallel, and therefore closed. The converse is also true: If $N_{J}=0$ and $d \omega=0$, then $D J=0$ (see [CMMS1, Thm 1]).

\subsection{Para-quaternionic Kähler manifolds}

DEFINITION 1.4.1. A para-quaternionic structure on a finite-dimensional vector space $V$ is a three-dimensional subalgebra $Q \subset \mathfrak{g l}(V)$ with a basis $I, J, K$ that satisfies the para-quaternionic relations

$$
I^{2}=-1, J^{2}=K^{2}=+1,-I J=J I=K .
$$

REMARK. From $-I J=J I$ we see that $I$ maps the +1 -eigenspace of $J$ isomorphically to the -1-eigenspace, so these eigenspaces have the same dimension. In other words, $J$ (as well as $K$ ) is a para-complex structure in the sense of Definition 1.1.1. 
Para-quaternionic vector spaces are identified with free right-modules over $\mathbf{H}$, where $\mathbf{H}=\mathbb{R} \oplus i \mathbb{R} \oplus j \mathbb{R} \oplus k \mathbb{R},-i^{2}=j^{2}=k^{2}=1$, ij= $-j i=k$, is the algebra of para-quaternions (also called split quaternions) and $I, J, K$ correspond to the multiplication by $i, j, k$ from the right. Accordingly, $\mathbf{H}^{n}$ is the space of para-quaternionic column vectors with scalar multiplication from the right. Multiplication by paraquaternionic matrices from the left is $\mathbf{H}$-linear.

The identification $\mathbf{H}=(\mathbb{R} \oplus i \mathbb{R}) \oplus j(\mathbb{R} \oplus i \mathbb{R})=\mathbb{C} \oplus j \mathbb{C}$ shows that any para-quaternionic vector space is a complex vector space of the double dimension. A map is $\mathbf{H}$-linear if and only if it is $\mathbb{C}$-linear and commutes with the right multiplication by $j$ (which is a $\mathbb{C}$-antilinear involution). In the same way, $\mathbf{H}=(\mathbb{R} \oplus j \mathbb{R}) \oplus i(\mathbb{R} \oplus j \mathbb{R})=\mathbf{C} \oplus i \mathbf{C}$ shows that any para-quaternionic vector space is a para-complex vector space of the double dimension. A map is $\mathbf{H}$-linear if and only if it is $\mathbf{C}$-linear and commutes with the right multiplication by $i$ (which is a $\mathbf{C}$-antilinear complex structure).

Definition 1.4.2. Let $(V, Q)$ be a para-quaternionic vector space. A pseudo-Euclidean metric $g$ on $V$ is said to be compatible with $Q$ or para-quaternion hermitian, if the elements of $Q$ are $g$-skew.

The standard para-quaternion hermitian metric on $\mathbf{H}^{n}$ is given by

$$
g\left(\left(h_{1}, \ldots, h_{n}\right),\left(h_{1}^{\prime}, \ldots, h_{n}^{\prime}\right)\right):=\operatorname{Re}\left(\sum \overline{h_{a}} h_{a}^{\prime}\right) .
$$

The isometry group

$$
\operatorname{Sp}^{\pi}(n):=\left\{A \in \mathrm{GL}(n, \mathbf{H}): A^{*} A=\mathrm{id}_{n}\right\}
$$

is called the para-symplectic group.

By the same argument as in Proposition 1.3.3, we see that any two para-quaternion hermitian vector spaces of the same dimension are isometric.

Proposition 1.4.3. $\operatorname{Sp}^{\pi}(n) \cong \operatorname{Sp}(2 n, \mathbb{R})$

PROOF. Let $\boldsymbol{\Omega}_{2 n}=\left(\begin{array}{cc}0 & -\mathbf{1}_{n} \\ \mathbf{1}_{n} & 0\end{array}\right)$ be the standard symplectic structure on $\mathbb{R}^{2 n}$, then $\mathbf{H}^{n} \cong\left(\mathbb{R}^{4 n}, I, J, K, g\right)$, where

$$
\begin{array}{cc}
I=\left(\begin{array}{cc}
0 & -\mathbf{1}_{2 n} \\
\mathbf{1}_{2 n} & 0
\end{array}\right) \quad J=\left(\begin{array}{cc}
\mathbf{1}_{2 n} & 0 \\
0 & -\mathbf{1}_{2 n}
\end{array}\right) \quad K=J I \\
g=-\omega_{I}(\cdot, I \cdot) \quad \omega_{I}=\left(\begin{array}{cc}
\Omega_{2 n} & 0 \\
0 & \Omega_{2 n}
\end{array}\right) .
\end{array}
$$

Thus, $\mathrm{Sp}^{\pi}(n)$ is identified with the endomorphism of $\mathbb{R}^{4 n}$ that commute with $I$ and $J$ and preserve $\omega_{I}$. This shows that

$$
\mathrm{Sp}^{\pi}(n) \cong\left\{\left(\begin{array}{cc}
A & 0 \\
0 & A
\end{array}\right): A \in \operatorname{Sp}(2 n, \mathbb{R})\right\} .
$$


DEFINITION 1.4.4. A para-quaternionic Kähler manifold $(M, g, Q)$ is a pseudo-Riemannian manifold $(M, g)$ endowed with a $g$-compatible para-quaternionic structure $Q \subset \operatorname{End}(T M)$ which is parallel with respect to the Levi-Civita connection.

It is not difficult to see (cf. [AC1, Prop. 1], [AC2, Prop. 9]) that the normalizer of $Q_{\mathbb{R}^{4 n}}=\mathbb{R} I+\mathbb{R} J+\mathbb{R} K$ in $\mathrm{O}(2 n, 2 n)$ is $G_{0} \cup J \cdot G_{0}$, where $G_{0}=\operatorname{Sp}^{\pi}(1) \cdot \operatorname{Sp}^{\pi}(n)$ is the subgroup of $\operatorname{GL}(4 n, \mathbb{R})=\operatorname{GL}_{\mathbb{R}}\left(\mathbf{H}^{n}\right)$ generated by left multiplication by elements of $\mathrm{Sp}^{\pi}(n)$ and right multiplication by elements of $\mathrm{Sp}^{\pi}(1)$ (which is just the group of unit paraquaternions), and where $J$ denotes the right multiplication by $j$ (which is an anti-unit, i. e. $\bar{j} j=-1$ ). So we have:

Proposition 1.4.5. A pseudo-Riemannian manifold is para-quaternionic Kähler if and only if

$$
\operatorname{Hol}(M) \subset\left(\mathrm{Sp}^{\pi}(1) \cdot \mathrm{Sp}^{\pi}(n)\right) \cup J \cdot\left(\mathrm{Sp}^{\pi}(1) \cdot \mathrm{Sp}^{\pi}(n)\right) .
$$

Note. In the definition in AC1, it is also required that $M$ be strongly oriented, i. e. that $\operatorname{Hol}(M) \subset \mathrm{SO}^{+}(2 n, 2 n)$. Of course, this is always fulfilled, if $M$ is simply connected.

It can be shown [AC1, Theorem 3] that any para-quaternionic Kähler manifold is Einstein and that its curvature tensor admits a decomposition

$$
R=\frac{\text { scal }}{4 n(n+2)} R_{0}+W
$$

where $R_{0}$ is the curvature tensor of $\mathbf{H P}^{n}$ and $W$ is an algebraic curvature tensor of type $\mathfrak{s p}^{\pi}(n)$. It follows that, if scal $\neq 0$, then $\mathfrak{s p}^{\pi}(1) \subset \mathfrak{h o l}$ (see [AC1, Corollary 1]). 



\section{CHAPTER 2}

\section{Para-Kähler submanifolds of para-quaternionic Kähler manifolds}

\subsection{Para-complex symplectic and contact structures}

The classical Darboux theorem states that all $2 n$-dimensional symplectic manifolds are locally isomorphic to $T^{*} \mathbb{R}^{n}$ equipped with the canonical symplectic form $d p_{a} \wedge d q^{a}$. As shown in this paragraph, the analogous statement in the para-holomorphic setting also holds.

\subsubsection{Para-complex symplectic vector spaces.}

Definition 2.1.1. Let $(V, J)$ be a para-complex vector space. A paracomplex symplectic form is an anti-symmetric C-bilinear map $\omega: V \times$ $V \rightarrow \mathbf{C}$ which is nondegenerate, i. e. $(\omega(X, \cdot)=0 \Leftrightarrow X=0)$.

Equivalently, a para-complex symplectic form is an element $\omega \in \wedge^{(2,0)} V^{*}$, such that the map $\omega: V^{(1,0)} \rightarrow \wedge^{(1,0)} V^{*}$ is an isomorphism.

Proposition 2.1.2. Let $(V, J)$ be a para-complex vector space and $\omega$ a para-complex symplectic form. Then there is a basis $\left\{Z^{a}, W_{b}\right\}_{a, b=1, \ldots, n}$ of the C-module $V$ such that $\omega\left(Z^{a}, W_{b}\right)=\delta_{b}^{a}, \omega\left(Z^{a}, Z^{b}\right)=0=\omega\left(W_{a}, W_{b}\right)$ for $a, b \in\{1, \ldots, n\}$.

Proof. Choose a regular vector $Z^{1} \in V$. We claim that there exists $W_{1} \in V$ such that $\omega\left(Z^{1}, W_{1}\right)=1$ : Otherwise the image of $W \mapsto \omega\left(Z^{1}, W\right)$ would be contained in one of the maximal ideals $(1 \pm j) \mathbb{R} \subset \mathbf{C}$. But then $\omega\left((1 \mp j) Z^{1}, \cdot\right) \equiv 0$, and therefore $(1 \mp j) Z^{1}=$ 0 by the nondegeneracy of $\omega$, in contradiction to the regularity of $Z^{1}$. From $\omega\left(Z^{1}, W_{1}\right)=1$ it now follows that $W_{1}$ is regular and that $Z^{1}$ and $W_{1}$ are C-linearly independent. Therefore, $\operatorname{span}_{\mathbf{C}}\left(Z^{1}, W_{1}\right)$ is a para-complex subspace, as well as the symplectic complement $\left(\operatorname{span}_{\mathbf{C}}\left(Z^{1}, W_{1}\right)\right)^{\perp_{\omega}}:=\left\{X \in V: \omega\left(Z^{1}, X\right)=\omega\left(W_{1}, X\right)=0\right\}$. The latter is again nondegenerate with respect to $\omega$, and the proposition follows by induction.

COROllary 2.1.3. An antisymmetic C-bilinear 2-form $\omega$ is nondegenerate if and only if $\omega^{n} \in \bigwedge^{(2 n, 0)} V^{*}$ is regular (where $n=\operatorname{dim}_{\mathbf{C}}(V) / 2$ ).

ProOF. If $\omega$ is nondegenerate and $\left\{Z^{a}, W_{b}\right\}_{a, b=1, \ldots, n}$ a basis as above, then $\omega^{n}\left(Z^{1}, W_{1}, \ldots, Z^{n}, W_{n}\right)=n$ !, in particular $(1 \pm j) \omega^{n} \neq 0$. 
Conversely, suppose that $\omega^{n}$ is regular. Let $\left\{B_{1}, \ldots, B_{2 n}\right\}$ be an arbitrary C-basis of $V$ and $X=\sum_{a} x^{a} B_{a} \in \operatorname{ker}(\omega), x^{a} \in \mathbf{C}$. We have to show that $X=0$. By the skew-symmetry of $\omega^{n}$ we have

$$
0=\omega^{n}\left(B_{1}, \ldots, B_{a-1}, X, B_{a+1}, \ldots, B_{2 n}\right)=x^{a} \cdot \omega^{n}\left(B_{1}, \ldots, B_{2 n}\right) .
$$

But by assumption we have $\omega^{n}\left(B_{1}, \ldots, B_{2 n}\right) \in \mathbf{C}^{*}$, so $x^{a}=0$.

\subsubsection{Para-holomorphic symplectic structures on manifolds.}

Definition 2.1.4. Let $(M, J)$ be a para-complex manifold. A paraholomorphic symplectic structure is a nondegenerate closed form $\omega \in$ $\Omega^{(2,0)} M$.

LEMMA 2.1.5. Let $(M, J)$ be a para-complex manifold and $\omega_{0}, \omega_{1}$ two para-holomorphic symplectic forms on $M$, which coincide in a point $p \in$ $M, \omega_{0}(p)=\omega_{1}(p)$. Then there is a para-holomorphic diffeomorphism $\Phi$ defined on a neighbourhood of $p$, such that $\Phi(p)=p$ and $\Phi^{*} \omega_{1}=\omega_{0}$.

We want to apply Moser's method of constructing $\Phi$ as time-1-map of a para-holomorphic flow, i. e. a flow $\Phi^{X}:(-\epsilon, \epsilon) \times U \rightarrow U$ of a (timedependent) vector field $X_{s}$, such that $\left(\Phi_{s}^{X}\right)^{*} J=J$ for any $s \in(-\epsilon, \epsilon)$. The vector field $X_{s}$ then needs to be an infinitesimal automorphism of the para-complex structure, i. e. $\mathcal{L}_{X_{s}} J=0$.

REMARK (on notation). If $X_{s}$ is a time-dependent vector field, then $\Phi_{s,}^{X}$. denotes the flow of $X$ starting at time $s$, i. e. $\frac{d}{d t} \Phi_{s, t}^{X}(p)=X\left(\Phi_{s, t}^{X}(p)\right)$, $\Phi_{s, s}^{X}(p)=p$. Instead of $\Phi_{0, s}^{X}$ we will also write $\Phi_{s}^{X}$. The Lie-derivative of a (possibly also time-dependent) tensor $T_{s}$ is defined as

$$
\mathcal{L}_{X_{s}} T_{s}:=\left.\frac{d}{d t}\right|_{t=0} \Phi_{s, s+t}^{*} T_{s+t}
$$

For functions, differential forms and vector fields this yields

$$
\begin{aligned}
\mathcal{L}_{X_{s}} f_{s} & =X_{s}\left(f_{s}\right)+\frac{\partial}{\partial s} f_{s} \\
\mathcal{L}_{X_{s}} \alpha_{s} & \left.\left.=d\left(X_{s}\right\lrcorner \alpha_{s}\right)+X_{s}\right\lrcorner d \alpha_{s}+\frac{\partial}{\partial s} \alpha_{s} \\
\mathcal{L}_{X_{s}} Y_{s} & =\left[X_{s}, Y_{s}\right]+\frac{\partial}{\partial s} Y_{s}
\end{aligned}
$$

Lemma 2.1.6. Let $(M, J)$ be a para-complex manifold. Then $\mathcal{L}_{X_{s}} J=0$ if and only if the $(1,0)$-vector field $\frac{1}{2}\left(X_{s}+j J X_{s}\right)$ is para-holomorphic.

ProOF. Using para-holomorphic coordinates $\left(z^{a}=x^{a}+j y^{a}\right)_{a=1 \ldots n}$ we write $X_{s}=\sum_{a} \xi_{s}^{a} \frac{\partial}{\partial x^{a}}+\sum_{a} \eta_{s}^{a} \frac{\partial}{\partial y^{a}}, \frac{1}{2}(X+j J X)=\sum\left(\xi_{s}^{a}+j \eta_{s}^{a}\right) \frac{\partial}{\partial z^{a}}$. 
The equations $J \frac{\partial}{\partial x^{a}}=\frac{\partial}{\partial y^{a}}$ and $J \frac{\partial}{\partial x^{a}}=\frac{\partial}{\partial y^{a}}$ yield

$$
\begin{aligned}
&\left(\mathcal{L}_{X_{s}} J\right)\left(\frac{\partial}{\partial x^{a}}\right)=\left[X_{s}, J \frac{\partial}{\partial x^{a}}\right]-J\left[X_{s}, \frac{\partial}{\partial x^{a}}\right] \\
&=\sum_{b}\left(\frac{\partial \eta_{s}^{b}}{\partial x^{a}}-\frac{\partial \xi_{s}^{b}}{\partial y^{a}}\right) \frac{\partial}{\partial x^{b}}+\left(\frac{\partial \xi_{s}^{b}}{\partial x^{a}}-\frac{\partial \eta_{s}^{b}}{\partial y^{a}}\right) \frac{\partial}{\partial y^{b}}
\end{aligned}
$$

The vanishing of this expression is equivalent to the para-CauchyRiemann equations for the function $\left(\xi_{s}^{b}+j \eta_{s}^{b}\right)$. The calculation for $\left(\mathcal{L}_{X} J\right)\left(\partial_{y_{k}}\right)$ is analogous.

REMARK 2.1.7. $\mathcal{L}_{X} J=0$ implies $\mathcal{L}_{J X} J=0$ and $[X, J X]=0$. If $X$ is time-independent, the flows $\Phi^{X}$ and $\Phi^{J X}$ commute, so $\Phi^{X}$ can be extended to a flow of $Z=\frac{1}{2}(X+j J X)$, depending on a paracomplex parameter $s+j t \in \mathbf{C}$, by $\Phi_{s+j t}^{Z}(p):=\Phi_{s}^{X}\left(\Phi_{t}^{J X}(p)\right)$, fulfilling $\frac{d}{d z} \Phi_{z}^{Z}(p)=Z\left(\Phi_{z}^{Z}(p)\right)$.

Proof of Lemma 2.1.5. Let $\omega_{t}=\omega_{0}+t\left(\omega_{1}-\omega_{0}\right), 0 \leq t \leq 1$. We have $\omega_{t}(p)=\omega_{0}(p)=\omega_{1}(p)$ for all $t$, so by the compactness of $[0,1]$ there is a neighbourhood of $p$, such that in this neighbourhood $\omega_{t}$ is nondegenerate for all $t \in[0,1]$. We will now construct a 1 -parameter family $\Phi_{t}$ of diffeomorphisms (defined on a neighbourhood of $p$ ), such that

$$
\begin{aligned}
\Phi_{t}(p) & =p \\
\Phi_{t}^{*} \omega_{t} & =\omega_{0} .
\end{aligned}
$$

Since $\omega^{\prime}:=\frac{d}{d t} \omega_{t}=\omega_{1}-\omega_{0}$ is closed by assumption, it locally is the differential of a 1-form, $\omega^{\prime}=d \alpha$. Further, we may assume that $\alpha(p)=0$ (by adding the differential of a function). Since $\bar{\partial}\left(\alpha^{(0,1)}\right)=\left(\omega^{\prime}\right)^{(0,2)}=0$, we can apply Corollary 1.2 .11 and find a (locally defined) function $\beta$, such that $\alpha^{(0,1)}=\bar{\partial} \beta$. Replacing $\alpha$ with $\alpha-d \beta$, we obtain $\alpha \in \Omega^{(1,0)} M$. Further, by construction, $\bar{\partial} \alpha=\omega^{\prime(1,1)}=0$, i. e. $\alpha$ is para-holomorphic. Thus the equation $\left.Z_{t}\right\lrcorner \omega_{t}=-\alpha$ defines a time-dependent vector field $Z_{t}$, which is para-holomorphic since $\alpha$ and $\omega_{t}^{-1}$ are, the latter by Cramer's rule. According to Remark 2.1.7, the flow of $X_{t}=2 \operatorname{Re}\left(Z_{t}\right)=Z_{t}+\bar{Z}_{t}$ is a 1-parameter family $\Phi_{t}$ of para-holomorphic diffeomorphisms. For all $t \in[0,1]$ we have $X_{t}(p)=0$, thus $\Phi_{t}(p)=p$, and $\Phi_{t}$ is defined on a neighbourhood of $p$. By writing $\mathcal{L}_{X}(U+j V)=[X, U+j V]$ and extending the definition of the Lie bracket and Lie derivative $\mathbf{C}$-linearly to the tensor algebra on $T^{\mathbf{C}} M$, we calculate (using Cartan's formula 
and $\left.\left.\bar{Z}_{t}\right\lrcorner \omega_{t}=0\right)$ :

$$
\begin{aligned}
\frac{d}{d t} \Phi_{t}^{*} \omega_{t} & \left.\left.=\Phi_{t}^{*}\left(d\left(X_{t}\right\lrcorner \omega_{t}\right)+X_{t}\right\lrcorner d \omega_{t}+\frac{d}{d t} \omega_{t}\right) \\
& \left.=\Phi_{t}^{*}\left(d\left(Z_{t}\right\lrcorner \omega_{t}\right)+0+\frac{d}{d t} \omega_{t}\right) \\
& =\Phi_{t}^{*}\left(-d \alpha+\omega^{\prime}\right)=0
\end{aligned}
$$

Integration then yields (2).

THEOREM 2.1.8. Let $(M, J)$ be a $2 n$-dimensional para-complex manifold and let $\omega$ be a para-holomorphic symplectic form. Then in the neighbourhood of any $p \in M$, there is a para-holomorphic coordinate system $\left(z^{a}, w_{b}\right)_{a, b=1, \ldots, n}$ such that $\omega=\sum_{a} d z^{a} \wedge d w_{a}$.

PROOF. In a sufficiently small neighbourhood of $p \in M$, choose an arbitrary para-holomorphic coordinate system $\left(\tilde{z}^{a}, \tilde{w}_{b}\right)$. From proposition 2.1.2 it follows that by changing the coordinates by a $\mathrm{C}$-linear map $\mathbf{C}^{n} \rightarrow \mathbf{C}^{n}$, we can arrange that $\omega(p)=\sum d \tilde{z}^{a}(p) \wedge d \tilde{w}_{a}(p)$. Now apply the above lemma to $\omega_{0}=\omega$ and $\omega_{1}=\sum d \tilde{z}^{a} \wedge d \tilde{w}_{a}$. Then $\left(z^{a}:=\tilde{z}^{a} \circ \Phi, w_{b}:=\tilde{w}_{b} \circ \Phi\right)$ is the desired coordinate system.

\subsubsection{Para-holomorphic contact forms.}

Definition. Let $M$ be a $(2 n+1)$-dimensional para-complex manifold. A para-holomorphic contact form is a para-holomorphic 1-form $\alpha \in$ $\Omega^{(1,0)}(M)$, such that $\left(\alpha \wedge(d \alpha)^{n}\right) \in \Omega^{(2 n+1,0)}(M)$ is regular.

REMARK. This condition is equivalent to $\operatorname{ker}(\alpha)$ being a $2 n$-dimensional para-holomorphic distribution in the sense of [AC2], chapter 2.2 (see [AC2, Proposition 3) and $\left.d \alpha\right|_{\operatorname{ker}(\alpha)}$ being nondegenerate (cf. Corollary 2.1.3.

LEMma 2.1.9. There is a unique vector field $R_{\alpha}$, called Reeb vector field, such that

$$
\begin{array}{r}
\left.R_{\alpha}\right\lrcorner d \alpha=0 \\
\left.R_{\alpha}\right\lrcorner \alpha=1
\end{array}
$$

Proof. At each $p \in M, d \alpha(p)$ has $\operatorname{rank} 2 n$, so $\operatorname{ker}(d \alpha(p))$ is 1 dimensional. With similar reasoning as in the proof of Proposition 2.1.2 there is a (unique) vector $R(p) \in \operatorname{ker}(d \alpha(p))$ which normalizes $\alpha(p)$ : Otherwise, either $(1+j) \alpha(p)$ or $(1-j) \alpha(p)$ would be zero on $\operatorname{ker}(d \alpha(p))$, and therefore $(1 \pm j) \alpha \wedge(d \alpha)^{n}=0$.

LEMMA 2.1.10. Let $M$ be a para-complex manifold and let $\alpha_{0}, \alpha_{1}$ be two para-holomorphic contact forms on $M$. Let $p \in M$ and $\alpha_{0}(p)=\alpha_{1}(p)$, $d \alpha_{0}(p)=d \alpha_{1}(p)$. Then there is a para-holomorphic diffeomorphism $\Phi$ defined on a neighbourhood of $p$, such that $\Phi(p)=p$ and $\Phi^{*} \alpha_{1}=\alpha_{0}$. 
ProOF. As in the proof of Lemma 2.1.5, let $\alpha_{t}=\alpha_{0}+t\left(\alpha_{1}-\alpha_{0}\right), 0 \leq$ $t \leq 1$. $\alpha_{t}$ is a contact form for all $t \in[0,1]$ in a neighbourhood of $p$. We will construct a 1-parameter familiy $\Phi_{t}$ of diffeomorphisms, such that

$$
\begin{aligned}
\Phi_{t}(p) & =p \\
\Phi_{t}^{*} \alpha_{t} & =\alpha_{0} .
\end{aligned}
$$

As before, if $\Phi_{t}$ is defined as the flow of a time-dependent vector field $X_{t}=2 \operatorname{Re}\left(Z_{t}\right)$, with $Z_{t}$ a para-holomorphic vectorfield to be specified below, then (3) follows from

$$
\left.\left.0=\frac{d}{d t} \Phi_{t}^{*} \alpha_{t}=Z_{t}\right\lrcorner d \alpha_{t}+d\left(Z_{t}\right\lrcorner \alpha_{t}\right)+\frac{d}{d t} \alpha_{t} .
$$

We decompose $Z_{t}=r_{t} R_{\alpha_{t}}+W_{t}$ with $W_{t}$ tangential to $\operatorname{ker}\left(\alpha_{t}\right)$ and obtain

$$
\left.W_{t}\right\lrcorner d \alpha_{t}+d r_{t}+\frac{d}{d t} \alpha_{t}=0 .
$$

Now $d \alpha_{t}$ is nondegenerate on $\operatorname{ker}\left(\alpha_{t}\right)$ and zero on $\operatorname{span}\left(R_{\alpha_{t}}\right)$, so for given $r_{t}$ there is a vector field $W_{t}$ which solves (4), if and only if $r_{t}$ satisfies

$$
\left.R_{\alpha_{t}}\right\lrcorner\left(d r_{t}+\frac{d}{d t} \alpha_{t}\right)=0 .
$$

This equation can be solved as follows: Let $\Psi_{t, z}$ denote the flow of $R_{\alpha_{t}}$ (see remark 2.1.7) and let $N$ be a $2 n$-dimensional para-complex submanifold of $M$ such that $p \in N$ and such that $N$ is transversal to $R_{\alpha_{t}}$ in a neighbourhood of $p$ (note that $R_{\alpha_{t}}(p)=R_{\alpha_{0}}(p)$, so we can take the same $N$ for all $t)$. Then $(z, q) \mapsto \Psi_{t, z}(q)$ is an isomorphism from a neighbourhood of $(0, p)$ in $\mathbf{C} \times N$ onto a neighbourhood of $p$ in $M$, and the equation (5) reads:

$$
\left.\frac{d}{d z} r_{t}\left(\Psi_{t, z}(q)\right)=-R_{\alpha_{t}}\left(\Psi_{t, z}(q)\right)\right\lrcorner\left(\frac{d}{d t} \alpha_{t}\right)
$$

The right hand side is para-holomorphic in $z$, so a solution for $r_{t}$ exists by Lemma 1.2.6. Moreover, by construction and because of $\frac{d}{d t} \alpha_{t}(p)=0$, we have $d r_{t}(p)=0=r_{t}(p)$, so that $Z_{t}(p)=0$, hence $\Phi_{t}(p) \stackrel{d t}{=}$, and $\Phi_{t}$ is well defined for all $t \in[0,1]$ in a neighbourhood of $p$.

THEOREM 2.1.11. Let $M$ be a $(2 n+1)$-dimensional para-complex manifold and let $\alpha$ be a para-holomorphic contact form. Then in the neighbourhood of any $p \in M$, there is a para-holomorphic coordinate system $\left(w_{0}, z^{a}, w_{b}\right)_{a, b=1, \ldots, n}$ such that $\alpha=d w_{0}-\sum w_{a} d z^{a}$.

PROOF. Let $\left(\tilde{w}_{0}, \tilde{z}^{a}, \tilde{w}_{b}\right)_{a, b=1, \ldots, n}$ be a coordinate system such that $\frac{\partial}{\partial \tilde{w}_{0}}(p)=R_{\alpha}(p)$ and such that $\left(\frac{\partial}{\partial \tilde{z}^{a}}(p), \frac{\partial}{\partial \tilde{w}_{b}}(p)\right)$ is a symplectic basis for $\left.d \alpha(p)\right|_{\operatorname{ker}(\alpha(p))}$. By adding a constant, we can assume that $p$ is mapped to zero. Then it follows that $\alpha(p)=\left(d \tilde{w}_{0}-\sum \tilde{w}_{a} d \tilde{z}^{a}\right)(p)$ and $d \alpha(p)=$ 
$\sum d \tilde{z}^{a}(p) \wedge d \tilde{w}_{a}(p)$. Now apply Lemma 2.1.10 to $\alpha_{0}=\alpha, \alpha_{1}=d \tilde{w}_{0}-$ $\sum \tilde{w}_{a} d \tilde{z}^{a}$ and set $z=\tilde{z} \circ \Phi, v^{a}=\tilde{v}^{a} \circ \Phi, w_{b}=\tilde{w}_{b} \circ \Phi$.

\subsection{Para-Kähler submanifolds and Twistor lift}

Let $(M, Q, g)$ be a para-quaternionic Kähler manifold.

Definition 2.2.1. The twistor bundle $\pi: Z \rightarrow M$ is the bundle of para-complex structures in $Q \subset \operatorname{End}(T M)$ :

$$
Z:=\left\{q \in Q: q^{2}=\mathrm{id}_{T M}\right\} .
$$

The fibres of $Z$ are one-sheeted hyperboloids: If $I_{p}, J_{p}, K_{p}$ is a basis for $Q_{p}$ as in definition 1.4.1, then $Z_{p}=\left\{r I_{p}+s J_{p}+t K_{p}:-r^{2}+s^{2}+t^{2}=1\right\}$. This can be identified with the homogenous space $\operatorname{SO}(2,1) / \mathrm{SO}(1,1)=$ $\mathrm{Sp}^{\pi}(1) / \mathrm{U}^{\pi}(1)$.

The Levi-Civita connection on $T M$ induces a splitting of $T Z$ into a vertical and a horizontal distribution $T Z=\mathcal{V} \oplus \mathcal{H}=\operatorname{ker}(d \pi) \oplus \mathcal{H}^{D}$. With respect to this splitting, we can define an almost complex structure $J_{Z}:=J_{\mathcal{V}}+J_{\mathcal{H}}$, where at each point $z \in Z, J_{\mathcal{H}}(z)$ is the para-complex structure given by $z \in Q_{\pi(z)} \subset \operatorname{End}\left(T_{\pi(z)} M\right) \cong \operatorname{End}\left(\mathcal{H}_{z}\right)$, and where $J_{\mathcal{V}}(z)$ is a $\mathrm{SO}(2,1)$-invariant para-complex structure on the homogenous space $Z_{\pi(z)}$ (see [AC2, Proposition 6]).

Likewise, we can define a one-parameter family $g_{Z}^{t}=t g_{\mathcal{V}}+g_{\mathcal{H}}, t \in$ $\mathbb{R} \backslash\{0\}$, of para-hermitian metrics, such that the map $\pi: Z \rightarrow M$ is a pseudo-Riemannian submersion (see [AC2, Proposition 7]), and where $-2 g_{\mathcal{V}}$ is the Killing form on $\mathfrak{s o}(2,1)$.

THEOREM 2.2.2. ([AC2, Theorem 2 and 3]) Let $Z$ be the twistor space of a para-quaternionic Kähler manifold with nonzero scalar curvature. Then

(1) The almost para-complex structure $J_{Z}$ is integrable.

(2) $g_{Z}^{-4 n(n+2) / s c a l}$ is a para-Kähler-Einstein metric.

(3) The horizontal distribution $\mathcal{H} \subset T Z$ is a para-holomorphic contact structure.

Definition 2.2.3. Let $(M, Q, g)$ be a para-quaternionic Kähler manifold. A para-complex submanifold of $M$ is a pair $(N, J)$, where $N \subset M$ is a smooth submanifold and $J$ a parallel section of $\left.Z\right|_{N}=\{q \in$ $\left.\left.Q\right|_{N}: q^{2}=1\right\}$, such that $J(T N)=T N$, and such that the $\pm 1-$ eigenspaces $T N^{ \pm}$have the same dimension. A para-complex submanifold is called para-Kähler submanifold, if the induced pseudoRiemannian metric $g_{N}:=\left.g\right|_{T N}$ is nondegenerate.

REMARK. It follows that the almost complex structure $J_{N}:=\left.J\right|_{T N}$ is integrable, since it is parallel with respect to a torsion-free connection. 
REMARK. Let $N \subset M$ be a para-Kähler submanifold. Then the map $f:\left(N, g_{N}\right) \rightarrow(M, g)$ is an isometric immersion which fulfills

$$
d f \circ J_{N}=J \circ d f
$$

where $J$ is a parallel section of $f^{*} Q \subset f^{*} \operatorname{End}(T M)$. Thus, in the language of section 3.2, the map $f$ is isotropic para-pluriharmonic, see equation (10) (with $\Phi_{\theta}:=e^{\theta J}$ ). Note that in section 3.2, $M$ is a symmetric space, but even without that assumption, equation (6) implies that $f$ is para-pluriharmonic: Because $J_{N}$ and $J$ are parallel, we have $(D d f) J_{N}=D\left(d f \cdot J_{N}\right)=D(J \cdot d f)=J(D d f)$, and therefore

$$
\begin{aligned}
& (D d f)\left(X, J_{N} Y\right)-(D d f)\left(Y, J_{N} X\right) \\
= & J((D d f)(X, Y)-(D d f)(Y, X))=0 .
\end{aligned}
$$

It follows that $(D d f)^{(+1,-1)}=0$, and that $f$ is para-pluriharmonic by Proposition 3.1.3.

The following theorem by Alekseevsky and Cortés relates the paracomplex submanifolds of a para-quaternionic Kähler manifold to the horizontal submanifolds of its twistor space:

THEOREM. [AC2, Theorem 4] Let $(M, Q, g)$ be a para-quaternionic Kähler manifold with nonzero scalar curvature and let $\pi: Z \rightarrow M$ be its twistor bundle.

(1) If $(N, J)$ is a para-complex submanifold of $M$, then $F: N \rightarrow$ $Z, F(p):=J_{p}$ defines an embedding of $N$ into $Z$ such that $\pi \circ F=\mathrm{id}_{N}$. The image $\tilde{N}=F(N)$ is a horizontal paracomplex submanifold of $Z$ (called the canonical lift of $N$ ).

(2) Conversely, if $\tilde{N} \subset Z$ is a para-complex horizontal submanifold such that $\pi: \tilde{N} \rightarrow \pi(\tilde{N}) \subset M$ is a diffeomorphism, then its projection $\left(N:=\pi(\tilde{N}), J:=d \pi \circ J_{Z} \circ d \pi^{-1}\right)$ is a para-complex submanifold of $M$.

(3) Moreover, $N$ is a para-Kähler submanifold, if and only if the one-parameter family of metrics $g_{Z}^{t}$ is nondegenerate on $\tilde{N} \subset$ $Z$.

Because the horizontal distribution $\mathcal{H} \subset T Z$ is a contact distribution, it follows that the dimension of $N$ cannot be greater than half the dimension of $M$. The maximal (i. e. of maximal dimension) para-complex submanifolds of $M$ correspond to Legendrian submanifolds (i. e. submanifolds tangent to the contact distribution with maximal possible dimension) of $Z$.

Combining this with the results of the preceding section, we can show that the maximal para-complex submanifolds are locally given by paraholomorphic functions: 
THEOREM 2.2.4. Let $M$ be a para-quaternionic Kähler manifold of real dimension $4 n$, and let $\pi: Z \rightarrow M$ be its twistor bundle.

Then at any point $q \in Z$ there exist local coordinates

$$
\left(z^{1}, \ldots z^{n}, w_{0}, \ldots, w_{n}\right): U \stackrel{\sim}{\rightarrow} U_{z} \times U_{w} \subset \mathbf{C}^{2 n+1}
$$

defined on a neighbourhood $U$ of $q$, such that the horizontal distribution is $\mathcal{H}=\operatorname{ker}\left(d w_{0}-\sum w_{a} d z^{a}\right)$.

For any para-holomorphic function $h: U_{z} \rightarrow \mathbf{C}$, the equations

$$
\begin{aligned}
& w_{0}=h\left(z^{1}, \ldots, z^{n}\right) \\
& w_{a}=\partial h / \partial z^{a}
\end{aligned}
$$

define a horizontal submanifold $\tilde{N} \subset Z$ (provided that $\left(w_{0}, \ldots, w_{n}\right) \in$ $\left.U_{w}\right)$, and the projection $\pi(\tilde{N})$ is a para-complex submanifold of $M$.

Any maximal para-complex submanifold $N \subset M$ is locally of this form with respect to a locally finite covering of $Z$ by such coordinate domains.

ProOF. Since $\mathcal{H}$ is a para-holomorphic contact distribution, the existence of local coordinates as above follows from Theorem 2.1.11. Since $Z$ is paracompact, it is locally finitely covered by such coordinates. Clearly, with respect to the contact form $d w_{0}-\sum w_{a} d z^{a}$, a section $U_{z} \rightarrow U_{z} \times U_{w}$ is horizontal, if and only if $w_{a}=\partial w_{0} / \partial z^{a}$. It remains to show that, given a horizontal $n$-dimensional submanifold $\tilde{N} \subset U$, we can write it as such a section. Let $\left(z^{1}, \ldots, z^{n}, w_{0}, \ldots, w_{n}\right)$ be a system of local coordinates as above. We may assume that $U_{z} \times U_{w}$ is a sufficiently small neighbourhood of $0 \in \mathbf{C}^{2 n+1}$, so that the $2 n$ differential forms $d z^{1}, \ldots d z^{n}, d w_{1}, \ldots, d w_{n}$ are pointwise C-linearly independent on $\left.\mathcal{H}\right|_{U}$. In other words, the $2 \cdot 2 n$ real-valued differential forms $d z_{ \pm}^{1}, \ldots, d z_{ \pm}^{n}$, $d w_{1}^{ \pm}, \ldots, d w_{n}^{ \pm}$, are $\mathbb{R}$-linearly independent on $\mathcal{H}^{ \pm} \subset T Z^{ \pm}$. Therefore a subset of $2 n$ of these forms ( $n$ for each sign) is $\mathbb{R}$-linearly independent on $T \tilde{N}^{ \pm}$. After exchanging some of the $z_{ \pm^{-}}$and $w^{ \pm}$-coordinates (note that $d w_{0}^{ \pm}-w^{ \pm} d z_{ \pm}=d\left(w_{0}^{ \pm}-w^{ \pm} z_{ \pm}\right)+z_{ \pm} d w^{ \pm}$), we can assume that $d z_{ \pm}^{1}, \ldots, d z_{ \pm}^{n}$ are linearly independent on $T \tilde{N}^{ \pm}$, so by the inverse function theorem, the $z$-coordinates form a local coordinate system on $\tilde{N}$.

2.2.1. Example: The twistor space $\mathrm{CP}^{2 n+1} \rightarrow \mathbf{H P}^{n}$. Let $\mathbf{C P}^{n}$ be the space of nondegenerate para-complex lines in $\mathbf{C}^{n+1}$,

$$
\mathbf{C P}^{n}=\left\{\left(z_{o}, \ldots, z_{n}\right) \in \mathbf{C}^{n+1}: \sum_{a=0}^{n} \bar{z}_{a} z_{a} \neq 0\right\} / \mathbf{C}^{*}
$$

Accordingly, let

$$
\mathbf{H P}^{n}=\left\{\left(h_{0}, \ldots, h_{n}\right) \in \mathbf{H}^{n+1}: \sum_{a=0}^{n} \bar{h}_{a} h_{a} \neq 0\right\} / \mathbf{H}^{*} .
$$


In each para-quaternionic line there is a unit element, which can be completed to a unitary basis of $\mathbf{H}^{n+1}$ (see the proof of Proposition 1.3.3), so $\mathrm{Sp}^{\pi}(n+1)$ acts transitively on $\mathrm{HP}^{n}$; therefore we can identify the latter with the coset space $\operatorname{Sp}^{\pi}(n+1) / \operatorname{Sp}^{\pi}(n) \times \operatorname{Sp}^{\pi}(1)$.

Consider the identification $\mathbf{H}^{n+1}=\mathbf{C}^{n+1} \oplus i \mathbf{C}^{n+1} \cong \mathbf{C}^{2 n+2}$ and note that $\overline{(z+i w)}(z+i w)=\bar{z} z+\bar{w} w$ for $z, w \in \mathbf{C}$, so the scalar products (and the notions of nondegeneracy) on $\mathbf{H}^{n+1}$ and $\mathbf{C}^{2 n+2}$ agree. In particular, there is a fibration $\mathrm{CP}^{2 n+1} \rightarrow \mathbf{H P}^{n}$, which is the fibration of homogenous spaces

$$
\frac{\mathrm{Sp}^{\pi}(n+1)}{\mathrm{Sp}^{\pi}(n) \times \mathrm{U}^{\pi}(1)} \rightarrow \frac{\mathrm{Sp}^{\pi}(n+1)}{\mathrm{Sp}^{\pi}(n) \times \mathrm{Sp}^{\pi}(1)}
$$

We want to give an explicit expression for the contact form: Let $\omega=$ $\sum_{a=0}^{n} d z^{a} \wedge d w_{a}$ be the standard symplectic form on $\mathbf{C}^{2 n+2}$ and let $X_{\text {rad }}=\sum_{a=0}^{n}\left(z_{a} \frac{\partial}{\partial z^{a}}+w_{a} \frac{\partial}{\partial w_{a}}\right)$ be the radial vector field. The 1-form $\left.\alpha^{\prime}:=X_{\mathrm{rad}}\right\lrcorner \omega=\sum_{a=0}^{n}\left(z^{a} d w_{a}-w_{a} d z^{a}\right)$ is homogenous of degree one and vanishes in the radial direction, so it yields a holomorphic form $\alpha$ on $\mathrm{CP}^{2 n+1}$ which is well-defined up to multiplication by a non-vanishing function. The kernel of $\alpha^{\prime}$ in $\mathbf{C}^{2 n+2}$ is the orthogonal complement $\left(X_{\mathrm{rad}} i\right)^{\perp}$ of the vertical vector field $X_{\text {rad }} i=i \sum_{a=0}^{n}\left(\bar{z}^{a} \frac{\partial}{\partial w_{a}}-\bar{w}_{a} \frac{\partial}{\partial z^{a}}\right)$, and thus $\operatorname{ker}(\alpha)$ is indeed the horizontal distribution in $\mathrm{CP}^{2 n+1}$.

On the subset $U_{0}:=\left\{\left[z^{0}, w_{0}, \ldots z^{n}, w_{n}\right]: z_{0} \in \mathbf{C}^{*}\right\} \subset \mathbf{C P}^{2 n+1}$ we introduce affine coordinates $\left[z^{0}, \ldots, w_{n}\right] \mapsto\left(z^{0}\right)^{-1}\left(w_{0}, z^{1}, \ldots, w_{n}\right)$, thereby identifying $U_{0}$ with the hyperplane $\left\{z^{0} \equiv 1\right\} \subset \mathbf{C}^{2 n+2}$. In these coordinates we have

$$
\left.\alpha\right|_{z^{0} \equiv 1}=d w_{0}+\sum_{a=1}^{n}\left(z^{a} d w_{a}-w_{a} d z^{a}\right)
$$

Coordinates $\left(\tilde{z}^{a}, \tilde{w}_{b}\right)$ as in Theorem 2.1.11 are given by

$$
\begin{aligned}
\tilde{w}_{0} & =w_{0}+\sum z^{a} w_{a} \\
\tilde{z}^{a} & =z^{a} \\
\tilde{w}_{a} & =2 w_{a} .
\end{aligned}
$$

Using these coordinates, we can construct the following horizontal immersions $\mathbf{C}^{n} \supset U \rightarrow \mathbf{C P}^{2 n+1}$ :

$$
\left(z^{1}, \ldots, z^{n}\right) \mapsto\left[1: h-\sum z^{a} \frac{\partial h / 2}{\partial z^{a}}: z^{1}: \frac{\partial h / 2}{\partial z^{1}}: \cdots: z^{n}: \frac{\partial h / 2}{\partial z^{n}}\right],
$$

where $h: U \rightarrow \mathbf{C}$ is a holomorphic function.

Accordingly, the para-complex immersions $U \rightarrow \mathbf{H P}^{n}$ are $\left(z^{1}, \ldots, z^{n}\right) \mapsto\left[1+i\left(h-\sum z^{a} \frac{\partial h / 2}{\partial z^{a}}\right): z^{1}+i \frac{\partial h / 2}{\partial z^{1}}: \cdots: z^{n}+i \frac{\partial h / 2}{\partial z^{n}}\right]$. 



\section{CHAPTER 3}

\section{Para-pluriharmonic maps into symmetric spaces}

\subsection{Para-pluriharmonic maps}

\subsubsection{Definitions.}

DeFinition 3.1.1. Let $\left(N, g_{N}\right),\left(M, g_{M}\right)$ be pseudo-Riemannian manifolds. A map $f: N \rightarrow M$ is called harmonic if it is a critical point of the energy functional under variations with compact support

$$
E(f):=\frac{1}{2} \int_{N} \operatorname{tr}_{g_{N}}\left(f^{*} g_{M}\right) d \operatorname{vol}_{N} .
$$

The first variation of the energy is given by

$$
\frac{d}{d t} E\left(f_{t}\right)=\int_{N} g_{M}\left(\frac{\partial f_{t}}{\partial t}, \operatorname{tr}_{g_{N}}(D d f)\right)+\operatorname{div} X d \operatorname{vol}_{N},
$$

where $D$ is the connection on $\operatorname{End}\left(T N, f^{*} T M\right)$ induced by the LeviCivita connections on $T N$ and $T M$ and where $X$ is the vector field on $N$ defined by $g_{N}(X, \cdot)=g_{M}\left(\frac{\partial f_{t}}{\partial t}, d f \cdot\right)$. Therefore, $f$ is harmonic if and only if

$$
\operatorname{tr}_{g_{N}}(D d f)=0
$$

If $N$ is 2-dimensional, then the energy functional is invariant under conformal changes of the metric $g_{N}$, so the definition depends only on the conformal class of $g_{N}$. But in two dimensions, the conformal classes of definite metrics correspond to the complex structures (up to sign), and the conformal classes of indefinite metrics correspond to the paracomplex structures (any indefinite scalar product in two dimensions has two null directions, which we take as the \pm 1 -eigenspaces of the para-complex structure). The former case leads to the definition of pluriharmonic maps, which have already been studied (see [BR, ET2]), the latter case to para-pluriharmonic maps:

Definition 3.1.2. Let $(N, J, g)$ be a para-Kähler manifold and let $\left(M, g_{M}\right)$ be a pseudo-Riemannian manifold. A smooth map $f: N \rightarrow M$ is called para-pluriharmonic if it is harmonic along every 1-dimensional para-complex submanifold.

Proposition 3.1.3. For a map $f: N \rightarrow M$ with $N, M$ as above the following conditions are equivalent:

(1) $f$ is para-pluriharmonic 
(2) $(D d f)^{(+1,-1)}=0$, i. e. Ddf vanishes on $T^{+} N \otimes T^{-} N$.

$\left(2^{\prime}\right)(D d f)^{(1,1)}=0$, i. e. $(D d f)^{\mathbf{c}}$ vanishes on $T^{(1,0)} N \otimes T^{(0,1)} N$.

Proof. $(2) \Rightarrow(1)$ : Let $L \subset N$ be a para-complex curve and $z_{ \pm}=x \pm y$ a pair of holomorphic coordinates on $L$. The Levi Civita connection on $L$ differs from the one on $N$ only by the (traceless) second fundamental form, and at any point $p \in L$ the pseudo-Riemannian metric $\left.g\right|_{T_{p} L}$ is proportional to $d z_{+} \vee d z_{-}=d x \otimes d x-d y \otimes d y$. So we have $\operatorname{tr}_{g}(D d f) \sim(D d f)\left(\frac{\partial}{\partial x}, \frac{\partial}{\partial x}\right)-(D d f)\left(\frac{\partial}{\partial y}, \frac{\partial}{\partial y}\right)=(D d f)\left(\frac{\partial}{\partial z_{+}}, \frac{\partial}{\partial z_{-}}\right)=0$.

$(1) \Rightarrow(2)$ : Any pair of vectors $Z_{ \pm} \in T_{p}^{ \pm} N$ is tangent to a paracomplex curve through $p$. The same calculation as before shows that $(D d f)\left(Z_{+}, Z_{-}\right) \sim \operatorname{tr}_{g}(D d f)=0$.

$(2) \Leftrightarrow\left(2^{\prime}\right)$ is immediate from $\vee^{(1,1)}(T N)=\vee^{(+1,-1)}(T N) \otimes \mathbf{C}$ (cf. Proposition 1.2.10.

3.1.2. A remark on complexification. We observe that if $I$ is a complex structure on $V$, then $I^{\mathbb{C}}$ is a $\mathbb{C}$-linear complex structure on $V \otimes \mathbb{C}$, and $J^{\mathbb{C}}=i \cdot I^{\mathbb{C}}$ is a para-complex structure. This leads to the idea to construct para-pluriharmonic maps in the following way: Start with a pluriharmonic map $f: N \rightarrow M$, extend this to map $f^{\mathbb{C}}: N^{\mathbb{C}} \rightarrow M^{\mathbb{C}}$ and then restrict it to an alternate real form $\tilde{N}$ of $N^{\mathbb{C}}$, such that $i I_{N}^{\mathbb{C}}$ is a para-complex structure of $T \tilde{N}$. - The problem with this is that one cannot expect the image $f(\tilde{N})$ to be contained in a totally real submanifold $\tilde{M}$ of $M^{\mathbb{C}}$.

To illustrate this, we consider $N=\mathbb{R}^{2}$ with the standard complex structure $I=\left(\begin{array}{cc}0 & -1 \\ 1 & 0\end{array}\right)$ and $M=\mathbb{R}:$ A function $f: \mathbb{R}^{2} \rightarrow \mathbb{R}$ is harmonic, if and only if

$$
d(d f \circ I)=0
$$

Then the complex analytic continuation $f^{\mathbb{C}}: \mathbb{C}^{2} \rightarrow \mathbb{C}$ of $f$ is harmonic and para-harmonic,

$$
d\left(d f^{\mathbb{C}} \circ I^{\mathbb{C}}\right)=d\left(d f^{\mathbb{C}} \circ i I^{\mathbb{C}}\right)=0 .
$$

Restricting this to $(\mathbf{C}, j) \cong\left(\mathbb{R} e_{1} \oplus i \mathbb{R} e_{2}, i I\right)$, we obtain a para-harmonic function

$$
\left.f^{\mathbb{C}}\right|_{\mathbb{R} e_{1} \oplus i \mathbb{R} e_{2}}: \mathbb{R} e_{1} \oplus i \mathbb{R} e_{2} \rightarrow \mathbb{C}
$$

However, the image of $f^{\mathbb{C}}\left(\mathbb{R} e_{1} \oplus i \mathbb{R} e_{2}\right)$ is usually not contained in a onedimensional real subspace, e.g. if $f(x, y)=x+y$, then $f(x, i y)=\mathbb{C}$. 
3.1.3. Pseudo-Riemannian symmetric spaces. Recall that a pseudo-Riemannian symmetric space is a pseudo-Riemannian manifold $M$, such that for each point $p \in M$ there exists an isometry $s_{p}: M \stackrel{\sim}{\rightarrow} M$ (the geodesic reflection) whose differential at $p$ is $d s_{p}(p)=-\mathrm{id}_{T_{p} M}$. We will always assume that $M$ is connected. It follows that the identity component of the isometry group $G:=\operatorname{Iso}_{0}(M)$ acts transitively on $M$. Therefore $M$ can be identified with the coset space $G / K$, where $K=\{g \in G: g \cdot o=o\}$ is the stabilizer subgroup of a chosen base point $o \in M$. The conjugation by $s_{o}$ is an involutive automorphism $\sigma: G \rightarrow G$, the Cartan involution. It holds that $G_{0}^{\sigma} \subset K \subset G^{\sigma}$, where $G^{\sigma}$ denotes the fixed-point set of $\sigma$. The differential $d \sigma_{e}: \mathfrak{g} \rightarrow \mathfrak{g}$ defines the $\operatorname{Ad}_{K}$-invariant Cartan decomposition $\mathfrak{g}=\operatorname{ker}\left(d \sigma_{e}-\mathrm{id}\right) \oplus \operatorname{ker}\left(d \sigma_{e}+\right.$ id) $=: \mathfrak{k} \oplus \mathfrak{m}$. In fact, $\mathfrak{k}$ is the Lie algebra of $K$, so the differential of $G \rightarrow M$ annihilates $\mathfrak{k}$ and maps $\mathfrak{m}$ isomorphically to $T_{o} M$.

Conversely, let $G$ be a Lie group, $\sigma$ an involution of $G, K \subset G$ a closed subgroup such that $G_{0}^{\sigma} \subset K \subset G^{\sigma}$ and suppose that there exists an $\operatorname{Ad}_{K}$-invariant pseudo-Euclidean scalar product on $\mathfrak{m}:=\operatorname{ker}\left(d \sigma_{e}+\mathrm{id}_{\mathfrak{g}}\right)$ (e.g. the Killing form, if it is nondegenerate) for which $d \sigma$ is an isometry. Then $M=G / K$ has the structure of a pseudo-Riemannian symmetric space with the geodesic reflection given by $s_{g K}(h K)=$ $g \sigma\left(g^{-1} h K\right)$. The group $G$ acts by isometries.

By saying that $M=G / K$ is a symmetric space, we will implicitly assume that the image of $G \rightarrow \mathrm{Iso}(M)$ contains $\operatorname{Iso}_{0}(M)$. (This is automatically the case if $G$ is semisimple.) Usually, we will also demand that the kernel of $G \rightarrow \operatorname{Iso}(M)$ be discrete, in other words, that the $G$-action on $M$ be almost effective.

The following facts hold (see e.g. [H], ['N], [E])

FACT 3.1.4. Let $G / K$ be a pseudo-Riemannian symmetric space with almost effective $G$-action. Then

(1) $[\mathfrak{k}, \mathfrak{k}] \subset \mathfrak{k},[\mathfrak{k}, \mathfrak{m}] \subset \mathfrak{m},[\mathfrak{m}, \mathfrak{m}] \subset \mathfrak{k}$.

(2) If $\mathfrak{g}$ is semisimple then $[\mathfrak{m}, \mathfrak{m}]=\mathfrak{k}$.

(3) $[[\mathfrak{m}, \mathfrak{m}], \mathfrak{m}] \subset \mathfrak{m}$. The isomorphism $\mathfrak{m} \stackrel{\sim}{\rightarrow} T_{o} M$ identifies the Lie triple product on $\mathfrak{m}$ with the Riemannian curvature tensor (possibly up to sign, depending on the definition of the latter).

(4) The group $g K g^{-1}$ acts on $T_{g K} M$ by linear isometries preserving $R$. Moreover, if $G$ is semisimple then the map $g K_{0} g^{-1} \rightarrow$ Aut $_{0}\left(T_{g K} M, g, R\right)$ is surjective. The same holds if $M$ is simply connected and the image of $G$ in $\operatorname{Iso}(M)$ contains $\operatorname{Iso}_{0}(M)$. O’N, Theorem 8.14]. 
3.1.4. Existence of associated families. Pluriharmonic maps into Riemannian symmetric spaces of non-positive or non-negative curvature are characterized by the fact that they have associated families. In our setting this is slightly different:

THEOREM 3.1.5. Let $N$ be a connected and simply connected paracomplex manifold and $M$ a pseudo-Riemannian symmetric space. The following conditions for a smooth map $f: N \rightarrow M$ are equivalent:

(1) $f$ has an associated family, i.e. there exists a 1-parameter family $\left(f_{\theta}, \Phi_{\theta}\right)_{\theta \in \mathbb{R}}$ of maps $f_{\theta}: N \rightarrow M$ and parallel bundle isomorphisms $\Phi_{\theta}: f_{\theta}^{*} T M \rightarrow f^{*} T M$ preserving the pseudoRiemannian metric $g^{M}$ and the curvature $R^{M}$, such that

$$
d f \circ e^{\theta J}=\Phi_{\theta} \circ d f_{\theta}
$$

(2) $f$ is para-pluriharmonic and

$$
\begin{aligned}
& R^{M}\left(d f\left(T^{+} N\right), d f\left(T^{+} N\right)\right)=0 \\
& R^{M}\left(d f\left(T^{-} N\right), d f\left(T^{-} N\right)\right)=0 .
\end{aligned}
$$

(2') $f$ is para-pluriharmonic and

$$
R^{M}\left(d f\left(T^{(1,0)} N\right), d f\left(T^{(1,0)} N\right)\right)=0 .
$$

Proof. The equivalence $(1) \Leftrightarrow(2)$ follows from the answer to a more general question, which is, roughly speaking:

Given a vector bundle $E \rightarrow N$, such that the fibres are isomorphic to those of $T M$, when is a bundle homomorphism $F: T N \rightarrow E$ the differential of a function $f: N \rightarrow M ?$

The answer is given in [ET1, Theorem 1]:

TheOREM (Eschenburg, Tribuzy). Let $N$ be a simply connected smooth manifold and let $M$ be a pseudo-Riemannian symmetric space with Levi-Civita connection $D^{M}$ and curvature tensor $R^{M}$. Let $E$ be a vector bundle over $N$, equipped with a connection $D$, a D-parallel fibre metric $g$ and a D-parallel triple product $R^{E}: \wedge^{2} E \rightarrow$ End $(E)$, such that there exists a linear isometry $\Phi_{0}: T_{o} M \stackrel{\sim}{\rightarrow} E_{p}$ for some fixed points $o \in M, p \in N$, such that $\Phi_{0}^{*} R_{p}^{E}=R_{o}^{M}$. Let $F: T N \rightarrow E$ be a vector bundle homomorphism. Then there exists a smooth map $f: N \rightarrow M$ and a parallel bundle isomorphism $\Phi: f^{*} T M \stackrel{\sim}{\rightarrow}$ E preserving $R$ and $g$ such that

if and only if

$$
F=\Phi \circ d f
$$

$$
\begin{aligned}
\left(D_{X} F\right)(Y)-\left(D_{Y} F\right)(X) & =0 \\
D_{X} D_{Y} \sigma-D_{Y} D_{X} \sigma-D_{[X, Y]} \sigma & =R(F X, F Y) \sigma
\end{aligned}
$$


for all vector fields $X, Y \in \mathrm{X}(N)$ and sections $\sigma$ of $E$, where in the first equation $D$ denotes the connection on $\operatorname{Hom}(T N, E)$ induced by $D$ and a torsion-free connection on $T N$.

Applying this Theorem to the bundle $E=f^{*} T M$ with the pull-back connection, metric and curvature tensor and to the map $F_{\theta}=d f \circ e^{\theta J}$, we see that a solution $\left(\Phi_{\theta}, f_{\theta}\right)$ of the equation $F_{\theta}=\Phi_{\theta} \circ d f_{\theta}$ exists if and only if the conditions (8) and (9) are fulfilled. We already know that they are fulfilled for $F_{0}=d f$. We consider the first equation: Since $D J=0$, we have $D F_{\theta}=D F \cdot e^{\theta J}$. Now, if $X$ and $Y$ are of the same type, say $X, Y \in T^{+} N$, then $e^{\theta J}$ is just multiplication by $e^{\theta}$, so equation (8) remains valid for all $\theta \in \mathbb{R}$. Thus, it has only be checked for vectors of different types; in this case it holds for all $\theta \in \mathbb{R}$, if and only if both terms are zero - but this is precisely the pluriharmonicity. A similar consideration can be done for the second equation: This time, if $X, Y$ are of different types, the factors $e^{\theta}$ and $e^{-\theta}$ cancel, so we have to test equation (9) with vectors $X, Y$ of the same type: Then the right hand side picks up the factor $e^{ \pm 2 \theta}$, so equation $(9$ ) can only be fulfilled for all $\theta \in \mathbb{R}$ if both sides are zero. But for $F_{0}=d f$, this is precisely the condition for the images of $T^{ \pm} N$ given in (2).

The equivalence $(2) \Leftrightarrow\left(2^{\prime}\right)$ follows immediately from $T^{(1,0)} N=(1+$ j) $T^{+} N \oplus(1-j) T^{-} N$.

REMARK 3.1.6. If $M$ is simply connected, then the solution $\left(\Phi_{\theta}, f_{\theta}\right)$ is unique up to isometries of $M$ ([ET1, Theorem 1]). Moreover, if $f$ is full (i. e. if the image of $f$ is not contained in a totally geodesic subspace of $M$ ), then $\Phi_{\theta}$ is determined by $f_{\theta}$; this follows from [ET1, Theorem $2]$.

REMARK 3.1.7. In the Riemannian case, the condition

$$
R^{M}\left(d f\left(T^{(1,0)} N\right), d f\left(T^{(1,0)} N\right)\right)=0
$$

actually follows from the pluriharmonicity, provided that the curvature tensor of $M$ (viewed as a symmetric bilinear form on $\wedge^{(1,1)} T M$ ) is semi-definite; therefore, if $M$ is a Riemannian symmetric space of nonpositive or nonnegative curvature, all pluriharmonic maps have associated families. In our case this is not true, as shown by the following counterexample:

Let $M$ be any symmetric space with nonzero curvature and let $U \subset M$ be an open subset diffeomorphic to $\mathbb{R}^{n}$. Let $N:=U \times U, J_{N}=\operatorname{id}_{T U} \times\left(-\mathrm{id}_{T U}\right)$ and let $g$ be a compatible metric. The projection on the first factor $f: N \stackrel{\pi_{1}}{\rightarrow} U \hookrightarrow M$ is obviously para-pluriharmonic since it depends only on the +-coordinates. But since $R^{M}\left(d f\left(T^{+} N\right), d f\left(T^{+} N\right)\right) \neq 0$ by assumption, $f$ does not have an associated family. 
REMARK 3.1.8. If $M=G / K$ is a para-Kähler symmetric space, then the conditions for the existence of an associated family (Theorem 3.1.5(2)) can be expressed in a very nice way; this has been discovered recently by Eschenburg and Quast [EQ]:

Suppose that $G$ is semisimple. We consider the standard immersion $J: M \rightarrow \mathfrak{g}$, which assigns to each point $p \in M$ the para-complex structure $J_{p}$, which is a derivation of the Lie triple algebra $\left(T_{p} M, R_{p}\right)$ and therefore given by an element of $\mathfrak{g}$ acting by the adjoint representation. From the parallelity of the endomorphism field $J$ we conclude that the image $J(M) \subset \mathfrak{g}$ is the adjoint orbit $\operatorname{Ad}_{G}(\xi), \operatorname{ad}_{\xi}:=J_{e K}$ (see the proof of Proposition 3.2.2). Let $\mathfrak{g}=\mathfrak{k} \oplus \mathfrak{m}$ be the Cartan decomposition of $\mathfrak{g}$. By definition, $\operatorname{ad}_{\xi}$ is a para-complex structure on $\mathfrak{m}$ and zero on $\mathfrak{k}$. In particular, $\mathfrak{k}$ coincides with the stabilizer subalgebra of $\xi$. It follows that the map $M \rightarrow \operatorname{Ad}_{G}(\xi) \subset \mathfrak{g}$ is a $G$-equivariant covering. The tangent and normal bundles of $J(M) \subset \mathfrak{g}$ are $\operatorname{Ad}_{G}(\mathfrak{m})$ and $\operatorname{Ad}_{G}(\mathfrak{k})$, respectively. At the point $\eta \in \operatorname{Ad}_{G}(\xi)$, the para-complex structure on $T_{\eta} J(M)$ is $\operatorname{ad}_{\eta}$.

Now let $f: N \rightarrow M$ be a map from a para-Kähler manifold $N$ into $M$. The differential of $\tilde{f}: N \rightarrow M \rightarrow \mathfrak{g}$ can be considered as a $\mathfrak{g}$-valued differential form on $N$. Let

$$
\gamma:=J_{M} \cdot d \tilde{f} \cdot J_{N}=\operatorname{ad}_{\tilde{f}}\left(d \tilde{f} \cdot J_{N}\right) \in \Omega^{1}(N ; \mathfrak{g}) .
$$

We claim that $f$ has an associated family if and only if $\gamma$ is closed [EQ, Theorem 5.1].

Proof. A short calculation shows that

$$
\begin{aligned}
d \gamma\left(X, J_{N} Y\right) & =\left[\tilde{f}, \nabla d \tilde{f}(X, Y)-\nabla d \tilde{f}\left(J_{N} X, J_{N} Y\right)\right] \\
& +[d \tilde{f}(X), d \tilde{f}(Y)]+\left[d \tilde{f}\left(J_{N} X\right), d \tilde{f}\left(J_{N} Y\right)\right]
\end{aligned}
$$

where $\nabla$ denotes the connection on $T^{*} N \otimes \mathfrak{g}$ defined by the Levi-Civita connection on $T N$. Since $\operatorname{ad}_{\tilde{f}}$ vanishes on the normal bundle, the expression in the first line takes values in the tangent bundle. It is zero for all $X, Y$, if and only if

$$
\begin{aligned}
0 & =\left(\nabla d \tilde{f}(X, Y)-\nabla d \tilde{f}\left(J_{N} X, J_{N} Y\right)\right)^{T} \\
& =\operatorname{Dd} \tilde{f}(X, Y)-\operatorname{Dd} \tilde{f}\left(J_{N} X, J_{N} Y\right),
\end{aligned}
$$

which is precisely the definition of $\tilde{f}$ being para-pluriharmonic.

The expression in the second line takes values in the normal bundle. It is zero for all $X, Y$, if and only if

$$
\begin{aligned}
0 & =[d \tilde{f}(X), d \tilde{f}(Y)]+\left[d \tilde{f}\left(J_{N} X\right), d \tilde{f}\left(J_{N} Y\right)\right] \\
& =R^{J(M)}(d \tilde{f}(X), d \tilde{f}(Y))+R^{J(M)}\left(d \tilde{f}\left(J_{N} X\right), d \tilde{f}\left(J_{N} Y\right)\right),
\end{aligned}
$$

which is equivalent the condition $R^{M}\left(d f\left(T^{ \pm} N\right), d f\left(T^{ \pm} N\right)\right)=0$. 
In [EQ this is used to generalize the Sym-Bobenko construction of surfaces in $\mathbb{R}^{3}$ with prescribed constant mean curvature from their (harmonic) Gauß map.

3.1.5. The Maurer-Cartan form. If $G$ is a matrix group, i.e. a closed subgroup of $\mathrm{GL}(\cdot, \mathbb{R})$, then there is an alternative approach to associated families using the Maurer-Cartan form. The following is completely analogous to the Riemannian case, see [DE] (note that our $\Phi_{\theta}$ is $\Phi_{\theta}^{-1}$ there).

Let $N_{0}$ be a contractible open subset of $N$. Then any map $f: N_{0} \rightarrow$ $M=G / K$ can be lifted to a map $F: N_{0} \rightarrow G$ such that $f=\pi \circ F$; this is called a framing of $f$. This framing is unique up to right multiplication by $K$-valued maps. Without loss of generality, we may assume that $p_{o} \in N_{0}$ is mapped to $o=e K \in M$, and that $F\left(p_{o}\right)=e$.

To the framing $F$ we assign the $\mathfrak{g}$-valued differential form

$$
\alpha:=F^{-1} d F \in \Omega^{1}\left(N_{0} ; \mathfrak{g}\right),
$$

the Maurer-Cartan form. It holds that $d \alpha+\alpha \wedge \alpha=0$.

Suppose that $f: N \rightarrow M=G / K$ fulfills the conditions of Theorem 3.1.5. Let $\left(\Phi_{\theta}, f_{\theta}\right)$ be the associated family of $f$. For any $p \in N, \Phi_{\theta}(p)$ is a linear isometry $T_{f_{\theta}(p)} M \rightarrow T_{f(p)} M$ which preserves $R$, therefore under the requirements of Fact $3.1 .4(4), \Phi_{\theta}(p)$ is given by an element of $G$.

We can easily check that

$$
F_{\theta}:=\Phi_{\theta}^{-1} F
$$

is a framing of $f_{\theta}$ with corresponding Maurer-Cartan form

$$
\alpha_{\theta}=F_{\theta}^{-1} d F_{\theta}=\alpha+F^{-1} \Phi_{\theta} d \Phi_{\theta}^{-1} F .
$$

From the parallelity of $\Phi_{\theta}$ it follows (cf. [DE, Lemma 1]) that the last term on the right hand side takes values in $\mathfrak{m} \subset \mathfrak{g}$ and that $\left(\alpha_{\theta}\right)_{\mathfrak{k}}=\alpha_{\mathfrak{k}}$ for any $\theta \in \mathbb{R}$ (where $\alpha=\alpha_{\mathfrak{k}}+\alpha_{\mathfrak{m}}$ due to the splitting $\mathfrak{g}=\mathfrak{k} \oplus \mathfrak{m}$ ). By definition, the component $\left(\alpha_{\theta}\right)_{\mathfrak{m}}$ is the horizontal lift of $d f_{\theta}$, up to left multiplication by elements of $G$, therefore from equation (7) we obtain

$$
\left(\alpha_{\theta}\right)_{\mathfrak{m}}=\alpha_{\mathfrak{m}} \circ e^{\theta J}=e^{\theta} \alpha_{\mathfrak{m}}^{(+1,0)}+e^{-\theta} \alpha_{\mathfrak{m}}^{(0,-1)} .
$$

For fixed $p \in N_{0}$, the map $\theta \mapsto F_{\theta}(p)$ can be considered as an element of the loop group

$$
\Lambda:=\{\gamma: \mathbb{R} \rightarrow G ; \gamma \text { smooth }\} .
$$

Thus, we have defined a map $\mathcal{F}: N_{0} \rightarrow \Lambda, \mathcal{F}(p)(\theta):=F_{\theta}(p)$ which satisfies

$$
\mathcal{F}^{-1} d \mathcal{F}=\alpha_{\mathfrak{k}}+e^{\theta} \alpha_{\mathfrak{m}}^{(+1,0)}+e^{-\theta} \alpha_{\mathfrak{m}}^{(0,-1)}
$$


this is called an extended framing. By construction, $\mathcal{F}$ is unique up to constant loops with values in $K$, hence the map $\tilde{\mathcal{F}}:=\pi \circ \mathcal{F}: N \rightarrow \Lambda / K$ is uniquely determined by $f$ and globally defined on $N$.

Conversely we can reconstruct the associated family from $\mathcal{F}$ : Let $F_{\theta}=$ $\mathcal{F}(\theta)$ and $f_{\theta}=\pi \circ F_{\theta}$. Then a short calculation shows that

$$
d f_{\theta} \circ e^{\theta J}=\left(F_{0} F_{\theta}^{-1}\right) \circ d f_{0}
$$

and that $\Phi_{\theta}:=F_{0} F_{\theta}^{-1}$ is parallel.

\subsection{Isotropic para-pluriharmonic maps}

We now want to focus our attention to the case where the family $\left(f_{\theta}\right)$ is constant in $\theta$. Such maps are called isotropic para-pluriharmonic maps. Equation 7 then reads

$$
d f \circ e^{\theta J}=\Phi_{\theta} \circ d f,
$$

where $\Phi_{\theta}$ is a parallel bundle automorphism of $f^{*} T M$ preserving $R$ and $g$.

We now assume that $f$ is $f u l l$, i. e. that the image is not contained in a totally geodesic subspace of $M$.

Proposition 3.2.1. Let $f: N \rightarrow M$ be a full isotropic para-pluriharmonic map. Then

(1) $(\Phi)_{\theta \in \mathbb{R}}$ is a one-parameter group of automorphisms of $f^{*} T M$, i. e. $\Phi_{\theta} \circ \Phi_{\theta^{\prime}}=\Phi_{\theta+\theta^{\prime}}$

(2) $f^{*} T M$ has a decomposition into parallel eigenbundles: $f^{*} T M=\bigoplus_{\lambda \text { odd }} E_{\lambda}$, where $\left.\Phi_{\theta}\right|_{E_{\lambda}}=e^{\theta \lambda}$

(3) The image of $d f$ is contained in $E_{-1} \oplus E_{+1}$, and $E_{-1} \oplus E_{+1}$ generates $f^{*} T M$ as Lie triple algebra.

(4) $g\left(E_{\lambda}, E_{\mu}\right)=0$ if $\lambda+\mu \neq 0$ and $\operatorname{dim} E_{\lambda}=\operatorname{dim} E_{-\lambda}$.

Proof. Let $E_{\lambda}:=\bigcap_{\theta \in \mathbb{R}} \operatorname{ker}\left(\Phi_{\theta}-e^{\theta \lambda}\right)$. From equation 100 we see immediately that $d f\left(T^{ \pm} N\right) \subset E_{ \pm 1}$. Let $E_{ \pm 1}^{\prime}$ be the smallest parallel subbundle of $f^{*} T M$ containing $d f\left(T^{ \pm} N\right)$, then $E_{ \pm 1}^{\prime} \subset E_{ \pm 1}$, because $\Phi_{\theta}$ commutes with the parallel translation on $f^{*} T M$. Now let $E \subset f^{*} T M$ be the bundle generated by $\left(E_{-1}^{\prime} \oplus E_{+1}^{\prime}\right)$ as a Lie triple algebra. By definition we have $\left[\left[E_{\lambda}, E_{\mu}\right], E_{\nu}\right] \subset E_{\lambda+\mu+\nu}$ and thus $E \subset \bigoplus_{\lambda \text { odd }} E_{\lambda}$. We claim that $E=f^{*} T M$ : In fact, because $E$ is parallel and $R$ invariant and because $f$ is full, this is the consequence of [ET1, Theorem 2]. This proves (2) and (3). (1) is an immediate consequence. Now (4) follows from the fact that $\Phi_{\theta}$ is an isometry of $f^{*} T M$ : We have $\left.g\right|_{E_{\lambda} \times E_{\mu}}=\left.\Phi_{\theta}^{*} g\right|_{E_{\lambda} \times E_{\mu}}=\left.e^{(\lambda+\mu) \theta} g\right|_{E_{\lambda} \times E_{\mu}}$, which obviously implies the first statement. But then, $g$ is an isomorphism $E_{\lambda} \stackrel{\sim}{\rightarrow} E_{-\lambda}^{*}$, which proves the equality of dimensions. 
Assume now that $G$ is semisimple or that $M$ is simply connected (and that the image of $G$ in $\operatorname{Iso}(M)$ contains $\left.\operatorname{Iso}_{0}(M)\right)$.

For each $p \in N, \Phi_{\theta}(p)$ is a one-parameter group of automorphisms of the Lie triple algebra $\left(T_{f(p)} M, R\right)$, so by Fact 3.1.4(4), it can be considered as a one-parameter subgroup of $g K^{-1}$ (where $g K=f(p)$ ). Its generator $\frac{d}{d \theta} \Phi_{\theta}(p)$ is a derivation of the $\left(T_{g K} M, R\right)$ and therefore given by the adjoint representation of an element of $\operatorname{Ad}_{g}(\mathfrak{k})$. This element is uniquely determined, if the action of $G$ on $M$ is almost effective.

Let $p_{o} \in N$ and choose $o=f\left(p_{o}\right)$ as the base point in $M$.

PROPOSITION 3.2.2.

Under the identification ad: $\operatorname{Ad}_{g}(\mathfrak{k}) \stackrel{\sim}{\rightarrow} \operatorname{Der}\left(T_{g K} M, g, R\right)$, the map

$$
F=\left.\frac{d}{d \theta}\right|_{\theta=0} \Phi_{\theta}
$$

takes values in the adjoint orbit $\operatorname{Ad}_{G}(\xi) \subset \mathfrak{g}$, where $\operatorname{ad}_{\xi}:=F\left(p_{0}\right)$.

Proof. Let $p \in N$ and let $\gamma:[0,1] \rightarrow N$ be a smooth path from $p_{o}$ to $p$. Recall that the left invariant distribution $g \mathfrak{m} \subset T_{g} G$ yields a connection on the $K$-principal bundle $G \rightarrow M$ which agrees with the Levi-Civita connection on $T M=G \times_{K} \mathfrak{m}$. Therefore the parallel translation along $f \circ \gamma$ is given by a horizontal curve $g(t)$ in $G$, such that $g K=f \circ \gamma, g(0)=e$ and $g^{\prime} \in g \mathfrak{m}$. It follows that $F(\gamma(t))=\operatorname{Ad}_{g(t)} \xi$, because both sides are parallel endomorphism fields along $f \circ \gamma$, which coincide at $t=0$.

From Proposition 3.2 .1 we see immediately that $\operatorname{ad}_{\xi}$ has odd integer eigenvalues on $\mathfrak{m} \cong T_{o} M$. Therefore, if $\mathfrak{g}$ is semisimple, $\operatorname{ad}_{\xi}$ has even integer eigenvalues on $\mathfrak{k}=[\mathfrak{m}, \mathfrak{m}]$, and the sum of the \pm 1 -eigenspaces generates all of $\mathfrak{g}$ as Lie algebra. Elements $\xi \in \mathfrak{g}$ with this property are called canonical:

\subsection{Canonical elements and twistor spaces}

Definition 3.3.1. Let $\mathfrak{g}$ be a real semisimple Lie algebra. An element $\xi \in \mathfrak{g}$ is called canonical, if

(1) $\mathfrak{g}$ is the sum $\mathfrak{g}=\bigoplus_{\lambda \in \mathbb{Z}} \mathfrak{g}_{\lambda}$ of $\operatorname{ad}_{\xi^{-}}$eigenspaces $\mathfrak{g}_{\lambda}:=\operatorname{ker}\left(\operatorname{ad}_{\xi}-\right.$ $\left.\lambda \mathrm{id}_{\mathfrak{g}}\right)$ with integer eigenvalues.

(2) $\mathfrak{g}_{-1} \oplus \mathfrak{g}_{1}$ generates $\mathfrak{g}$ as Lie algebra.

Some authors replace the condition (2) by

$\left(2^{\prime}\right)$ the Lie algebra generated by $\mathfrak{g}_{-1} \oplus \mathfrak{g}_{1}$ contains $\bigoplus_{\lambda \neq 0} \mathfrak{g}_{\lambda}$.

To distinguish these notions, we call an element $\xi$ semi-canonical, if

(1) and (2') hold. 
LEMMA 3.3.2. If $\mathfrak{g}$ is simple, then any semi-canonical element of $\mathfrak{g}$ is either canonical or zero.

More generally, let $\mathfrak{g}$ be semisimple and let $\mathfrak{g}=\mathfrak{g}^{\prime} \oplus \mathfrak{g}^{\prime \prime} \oplus \cdots$ be the decomposition into simple summands. Then a semi-canonical element $\xi=\xi^{\prime}+\xi^{\prime \prime}+\cdots$ is canonical, if and only if all the $\xi^{\prime}, \xi^{\prime \prime}, \ldots$ are nonzero.

Proof. Let $\mathfrak{g}$ be simple and $\xi$ semi-canonical. From (2') and $\left[\mathfrak{g}_{0}, \mathfrak{g}_{ \pm 1}\right] \subset \mathfrak{g}_{ \pm 1}$ and the Jacobi identity it follows that the Lie algebra generated by $\mathfrak{g}_{-1} \oplus \mathfrak{g}_{1}$ is an ideal in $\mathfrak{g}$ and therefore either all of $\mathfrak{g}$ or trivial. This proves the first statement. The rest is straightforward.

Definition 3.3.3. Let $\xi \in \mathfrak{g}$ be semi-canonical. The adjoint orbit $Z(\xi):=\operatorname{Ad}_{G}(\xi)$ is called the twistor space associated to $\xi$.

FACT. $Z$ is the homogenous space $G / H$, where $H=\left\{g \in G: \operatorname{Ad}_{g}(\xi)=\xi\right\}$ is the centralizer of $\xi$ with Lie algebra $\mathfrak{h}=\mathfrak{g}_{0}$.

Proposition 3.3.4. Let $G$ be a connected real form of a complex semisimple Lie group. Let $\mathfrak{g}$ be the Lie algebra of $G$ and $\xi \in \mathfrak{g}$ semicanonical.

(1) The map $\sigma: g \mapsto e^{i \pi \xi} g e^{-i \pi \xi}\left(i^{2}=-1\right)$ is an involutive automorphism of $G$ with corresponding Cartan decomposition

$$
\mathfrak{g}=\mathfrak{k} \oplus \mathfrak{m}=\left(\bigoplus_{\lambda \text { even }} \mathfrak{g}_{\lambda}\right) \oplus\left(\bigoplus_{\lambda \text { odd }} \mathfrak{g}_{\lambda}\right) .
$$

(2) It holds that $H \subset G^{\sigma}$, and $H \cdot G_{0}^{\sigma}$ is a subgroup of $G$. Let $K$ be a subgroup of $G$ such that $H \cdot G_{0}^{\sigma} \subset K \subset G^{\sigma}$. Then there is a $G$-equivariant projection map $\pi: Z \rightarrow M$ onto the symmetric space $M=G / K$, called the twistor fibration.

(3) The $G$-action on $M$ is almost effective (i. e. the kernel of the map $G \rightarrow \operatorname{Iso}(M)$ is discrete) if and only if $\xi$ is canonical.

Proof. (1): The stated Cartan decomposition follows from the fact that $d \sigma=e^{i \pi \mathrm{ad}_{\xi}}$ is +1 on $\mathfrak{g}_{\lambda}$ if $\lambda$ is even, and -1 if $\lambda$ is odd. This also shows that $d \sigma(\mathfrak{g})=\mathfrak{g}$ and $d \sigma^{2}=\mathrm{id}$, which implies $\sigma(G)=G$ and $\sigma^{2}=\mathrm{id}$, since $G$ is connected.

(2): If $g \in H$, then $e^{i \pi \xi}=e^{i \pi \operatorname{Ad}_{g} \xi}=g e^{i \pi \xi} g^{-1}$, so $g \in G^{\sigma}$. This shows that $H \subset G^{\sigma}$. It now follows that $h G_{0}^{\sigma} h^{-1}=G_{0}^{\sigma}$ for any $h \in H$. Therefore $H \cdot G_{0}^{\sigma}=G_{0}^{\sigma} \cdot H$, which implies that $H \cdot G_{0}^{\sigma}$ is a subgroup of $G$. The rest is straightforward.

(3): Let $\xi$ be canonical. We have to show that the adjoint action $\mathfrak{k} \rightarrow \mathfrak{g l}(\mathfrak{m})$ is effective. Suppose that $X \in \mathfrak{k}$ and $\left.\operatorname{ad}_{X}\right|_{\mathfrak{m}}=0$. Since $\mathfrak{m} \supset$ $\mathfrak{g}_{-1} \oplus \mathfrak{g}_{1}$ generates $\mathfrak{g}$, it follows by the Jacobi identity that $\left.\operatorname{ad}_{X}\right|_{\mathfrak{g}}=0$, hence $X=0$. Vice versa, let $\xi$ be semi-canonical, but not canonical. Then the orthogonal complement (with respect to the Killing form) of 
the subalgebra generated by $\mathfrak{g}_{-1} \oplus \mathfrak{g}_{1}$ is an ideal contained in $\mathfrak{g}_{0} \subset \mathfrak{k}$ and therefore in the kernel of ad: $\mathfrak{k} \rightarrow \mathfrak{g l}(\mathfrak{m})$.

At $\xi \in Z$, the tangent space of $Z$ is $T_{\xi} Z=\operatorname{ad}_{\mathfrak{g}}(\xi)=\bigoplus_{\lambda \in \mathbb{Z} \backslash\{0\}} \mathfrak{g}_{\lambda}=$ : $\mathfrak{z}$. This eigenspace decomposition is $\operatorname{Ad}_{H}$-invariant and thus defines a decomposition of $T Z$ into $\operatorname{Ad}_{G}$-invariant distributions $\operatorname{Ad}_{G}\left(\mathfrak{g}_{\lambda}\right), \lambda \in$ $\mathbb{Z} \backslash\{0\}$.

Proposition 3.3.5. The twistor space $Z=\operatorname{Ad}_{G}(\xi)$ has a canonical para-complex structure which is given by multiplication by $\operatorname{sgn}(\lambda)$ on $\operatorname{Ad}_{G}\left(\mathfrak{g}_{\lambda}\right) \subset T Z$.

PROOF. The proposition follows from the fact that $Z$ can be written as a quotient of para-complex groups (see below). However, there is a direct proof: From the fact that $e^{\operatorname{ad}_{\xi}}$ is an isometry with respect to the (nondegenerate) Killing form it follows that $\operatorname{dim}\left(\mathfrak{g}_{\lambda}\right)=$ $\operatorname{dim}\left(\mathfrak{g}_{-\lambda}\right)$, cf. the proof of 3.2 .1 (4). Moreover, the distributions $T^{ \pm} Z=$ $\operatorname{Ad}_{G}\left(\bigoplus_{\lambda \gtrless 0} \mathfrak{g}_{\lambda}\right)$ are integrable, because they are tangent to the leaves $\operatorname{Ad}_{g N_{ \pm}}(\xi) \subset Z$, where $N_{ \pm}:=\exp \left(\bigoplus_{\lambda \gtrless 0} \mathfrak{g}_{\lambda}\right)$.

THEOREM 3.3.6. Let $N$ be a connected para-Kähler manifold and let $G$ be a connected real form of a semisimple complex Lie group.

(1) Let $M=G / K$ be a symmetric space with $K=G^{\sigma}$ and suppose that $G$ acts almost effectively. Then any full isotropic parapluriharmonic map $f: N \rightarrow M$ defines a canonical element $\xi \in \mathfrak{g}$ and a twistor fibration $\pi: Z \rightarrow M$. The map $f$ has a para-holomorphic lift $F: N \rightarrow Z, f=\pi \circ F$, such that the differential $d F$ takes values in the subbundle $\mathcal{H}_{1}:=\operatorname{Ad}_{G}\left(\mathfrak{g}_{1} \oplus\right.$ $\left.\mathfrak{g}_{-1}\right) \subset T Z$ (called superhorizontal bundle)

(2) Let $\xi \in \mathfrak{g}$ be canonical and let $\pi: Z \rightarrow M=G / K$ a twistor fibration as in Proposition 3.3.4. For any para-holomorphic superhorizontal map $F: N \rightarrow Z$ the map $f:=\pi \circ F$ is isotropic para-pluriharmonic.

Proof. For the proof of (1), there is only left to show that $F=$ $\left.\frac{d}{d \theta}\right|_{\theta=0} \Phi_{\theta}$ is para-holomorphic and superhorizontal, i. e. that $d F$ maps $T^{ \pm} N$ to $\operatorname{Ad}_{G}\left(\mathfrak{g}_{ \pm 1}\right)$ : In the proof of Proposition 3.2.2 we have already seen that $F \circ \gamma(t)=\operatorname{Ad}_{g(t)} \xi$ with $g^{-1} g^{\prime} \in \mathfrak{m}$ and therefore $d F$. $\gamma^{\prime} \in \operatorname{Ad}_{g}[\mathfrak{m}, \xi]=\operatorname{Ad}_{g}\left(\bigoplus_{\lambda \text { odd }} \mathfrak{g}_{\lambda}\right)$. The map $d \pi: T Z \rightarrow T M$ takes $\operatorname{Ad}_{G}\left(\mathfrak{g}_{\lambda}\right)$ to $E_{\lambda}$, and from equation 10 it follows that $d \pi \circ d F\left(T^{ \pm} N\right)=$ $d f\left(T^{ \pm} N\right) \subset E_{ \pm 1}$, hence $d F\left(T^{ \pm} N\right) \subset \operatorname{Ad}_{G}\left(\mathfrak{g}_{ \pm 1}\right)$.

In order to prove $(2)$, recall that the para-complex structure on the subspace $\operatorname{Ad}_{g}\left(\mathfrak{g}_{-1} \oplus \mathfrak{g}_{1}\right) \subset T_{\xi^{\prime}} Z, \xi^{\prime}=\operatorname{Ad}_{g} \xi$, is just ad $\left(\xi^{\prime}\right)$. From the fact that $F$ is holomorphic and the image of $d F$ is contained in $\operatorname{Ad}_{G}\left(\mathfrak{g}_{-1} \oplus\right.$ $\left.\mathfrak{g}_{1}\right)$, we obtain $d F \circ e^{\theta J}=e^{\theta \operatorname{ad}_{F}} \circ d F$. Composing this equation with $d \pi: T Z \rightarrow T M$ (which is multiplication by (-1) on $\operatorname{Ad}_{g}\left(\mathfrak{g}_{\lambda}\right), \lambda$ odd), and 
recalling that the isomorphism $\operatorname{Der}\left(\operatorname{Ad}_{g}(\mathfrak{m}),[[]],\right) \stackrel{\sim}{\rightarrow} \operatorname{Der}\left(T_{g K} M, g, R\right)$ identifies $\operatorname{ad}_{F}$ with $F$, we obtain $d f \circ e^{\theta J}=e^{\theta F} \circ d f$, where $e^{\theta F}=: \Phi_{\theta}$ is an automorphism field of $f^{*} T M$, which preserves the curvature tensor and any $\mathrm{Ad}_{K}$-invariant metric. $F$ and $\Phi_{\theta}$ are parallel, since $d F$ has values in the horizontal bundle $\operatorname{Ad}_{G}(\mathfrak{m}$ ) (see the proof of Proposition 3.2.2). Thus, $f=\pi \circ F$ is isotropic para-pluriharmonic by the definition.

Corollary 3.3.7. A necessary condition for the existence of full isotropic para-pluriharmonic maps $N \rightarrow M=G / G^{\sigma}$ is that $\sigma$ is of the form $\sigma(g)=e^{i \pi \xi} g e^{-i \pi \xi}$ for $\xi \in \mathfrak{g}$.

EXAMPLE 3.3.8. (para-holomorphic maps)

The easiest example of all this is as follows: Assume that $M=G / G^{\sigma}$ is a para-Kähler symmetric space with para-complex structure $J_{M}$. Any para-holomorphic map $f: N \rightarrow M$ is isotropic para-pluriharmonic, since $d f \circ e^{\theta J_{N}}=e^{\theta J_{M}} \circ d f$. The canonical element $\xi \in \mathfrak{g}$ for this map is exactly the para-complex structure $J_{M}$; it defines a depth-1 grading $\mathfrak{g}=\mathfrak{g}_{-1} \oplus \mathfrak{g}_{0} \oplus \mathfrak{g}_{1}$. By definition, $\xi$ is invariant under $G^{\sigma}$, so $G^{\sigma} \subset H$. We have already seen that $H \subset G^{\sigma}$, therefore $Z=M$, and the twistor fibration is just the identity map.

3.3.1. Para-complex description of the twistor space. We want to give a description of $Z$ as a quotient of para-complex groups:

Definition 3.3.9. Let $G$ be a (not necessarily semisimple) Lie group. The para-complexification of $G$ is the product $G^{\mathbf{c}}:=G \times G$. This is a para-complex manifold, with the para-complex structure given by $J=\mathrm{id}_{T G} \times\left(-\mathrm{id}_{T G}\right)$. Its Lie algebra is $\mathfrak{g}^{\mathbf{c}}=\mathfrak{g} \times \mathfrak{g} \cong \mathfrak{g} \otimes \mathbf{C},(X, Y) \cong$ $\frac{1+j}{2} X+\frac{1-j}{2} Y$. (cf. Example 1.1.5). We can identify $G$ with the diagonal subgroup $\{(g, g): g \in G\}$, being the fixed-point set of the anti-holomorphic involution $(g, h) \mapsto(h, g)$ of $G^{\mathbf{c}}$.

Let $G$ be semisimple and $\xi \in \mathfrak{g}$ semi-canonical. There is a decomposition of $\mathfrak{g}^{\mathbf{c}}$ into $\operatorname{ad}_{(\xi, \xi)}$-eigenspaces with imaginary eigenvalues (see remark 1.1.3): For $\lambda \in \mathbb{Z}$, let $\mathfrak{g}_{\lambda}^{\mathbf{c}}:=\mathfrak{g}_{\lambda} \times \mathfrak{g}_{-\lambda}$. Then $\mathfrak{g}_{\lambda}^{\mathbf{c}}$ is the $\lambda j$ eigenspace of $\operatorname{ad}_{(\xi, \xi)}(j$ equals +1 on $\mathfrak{g} \times\{0\}$ and -1 on $\{0\} \times \mathfrak{g})$, and we have $\mathfrak{g}^{\mathbf{c}}=\bigoplus_{\lambda \in \mathbb{Z}} \mathfrak{g}_{\lambda}^{\mathbf{c}}$. We define the following subalgebras of $\mathfrak{g}$ and $\mathfrak{g}^{\mathbf{c}}$ :

$$
\begin{array}{cc}
\mathfrak{n}_{ \pm}:=\bigoplus_{\lambda \gtrless 0} \mathfrak{g}_{\lambda} \quad \mathfrak{n}_{ \pm}^{\mathbf{c}}:=\mathfrak{n}_{ \pm} \times \mathfrak{n}_{\mp} \\
\mathfrak{p}_{ \pm}:=\mathfrak{g}_{0} \oplus \mathfrak{n}_{ \pm} & \mathfrak{p}_{ \pm}^{\mathbf{c}}:=\mathfrak{p}_{ \pm} \times \mathfrak{p}_{\mp}
\end{array}
$$

The $\mathfrak{p}_{ \pm}$are parabolic subalgebras (see below) with nilradical (maximal nilpotent ideal) $\mathfrak{n}_{ \pm}$. Let

$$
N_{ \pm}:=\exp \left(\mathfrak{n}_{ \pm}\right), \quad P_{ \pm}:=\left\{g \in G: \operatorname{Ad}_{g} \xi \in \xi+\mathfrak{n}_{ \pm}\right\} .
$$

These are closed subgroups with respective Lie algebras $\mathfrak{n}_{ \pm}$and $\mathfrak{p}_{ \pm}$(see [ET2]). Let $P_{ \pm}^{\mathrm{c}}:=P_{ \pm} \times P_{\mp}$ and $N_{ \pm}^{\mathrm{c}}:=N_{+} \times N_{-}$. 
Proposition 3.3.10. $Z=G / H$ is an open subset of $\bar{Z}:=G^{\mathbf{c}} / P_{-}^{\mathbf{c}}=$ $\left(G / P_{-}\right) \times\left(G / P_{+}\right)$, called the closed twistor space. The para-complex structure on $Z$ inherited from $\bar{Z}$ coincides with the one given in proposition 3.3.5.

Proof. The map $G \stackrel{\Delta}{\hookrightarrow} G^{\mathbf{c}} \rightarrow G^{\mathbf{c}} / P_{-}^{\mathbf{c}}$ has kernel $P_{-} \cap P_{+}=H$, so we have a well-defined embedding $G / H \rightarrow G^{\mathbf{c}} / P_{-}^{\mathbf{c}}$. The differential of this map is the isomorphism $\mathfrak{g} / \mathfrak{g}_{0} \stackrel{\sim}{\rightarrow} \mathfrak{g}^{\mathbf{c}} / \mathfrak{p}_{-}^{\mathbf{c}} \cong \mathfrak{n}_{+} \times \mathfrak{n}_{-}$which sends $\mathfrak{g}_{\lambda}$ to $\mathfrak{g}_{\lambda} \times\{0\}$ or to $\{0\} \times \mathfrak{g}_{\lambda}$, depending on the sign of $\lambda$.

3.3.2. Parabolic subgroups. In order to describe the coset spaces $G / P_{\mp}$, we need some facts concerning (real) parabolic subgroups. We start with some basic definitions from algebraic geometry. (All of the following can be found in $[\mathrm{Bo}]$.)

An affine algebraic variety is a subset $V \subset \mathbb{C}^{n}$ which is the set of zeroes of a family of polynomials. It can be endowed with the Zariski topology, in which the closed sets are by definition the subvarieties.

Let $I(V) \subset \mathbb{C}\left[\mathbb{C}^{n}\right]$ be the ideal of polynomials vanishing on $V$. Then $\mathbb{C}[V]:=\mathbb{C}\left[\mathbb{C}^{n}\right] / I(V)$ is the ring of regular functions. A morphism of varieties is a map $\phi: V \rightarrow V^{\prime}$, such that $\phi^{*}\left(\mathbb{C}\left[V^{\prime}\right]\right) \subset \mathbb{C}[V] . V$ is said to be irreducible, if $V$ is not the proper union of two nonempty varieties, which is equivalent to $I(V)$ being a prime ideal. If $V$ is irreducible, then the quotient field of $\mathbb{C}[V]$ is called the field of rational functions, denoted by $\mathbb{C}(V)$.

A variety $V$ is said to be defined over $\mathbb{R}$, or an $\mathbb{R}$-variety, if $I(V)$ is generated by polynomials with real coefficients. In this case, the set $V(\mathbb{R})$ of real points of $V$ (i. e. the points of $V$ with real coordinates) is a real variety. A morphism $\phi: V \rightarrow V^{\prime}$ of $\mathbb{R}$-varieties is defined over $\mathbb{R}$ (or an $\mathbb{R}$-morphism), if $\phi^{*}\left(\mathbb{R}\left[V^{\prime}\right]\right) \subset \mathbb{R}[V]$.

A group is called linear algebraic, if it is both a subgroup and a subvariety of $\mathrm{GL}_{n}(\mathbb{C})$ (where $\mathrm{GL}_{n}(\mathbb{C})$ is the variety $\left\{\left(g, \operatorname{det}(g)^{-1}\right): g \in\right.$ $\left.\left.\mathrm{GL}_{n}(\mathbb{C})\right\} \subset \mathbb{C}^{n^{2}+1}\right)$. It follows that any linear algebraic group over $\mathbb{C}$ is a complex Lie group. If $G$ is an $\mathbb{R}$-group (i.e. a linear algebraic group defined over $\mathbb{R}$ ), then $G(\mathbb{R})$ is a real Lie group and a real form of $G$. Important examples of linear algebraic groups are the classical (real and complex) matrix groups. In fact, any connected semisimple complex Lie group is linear algebraic, as well as the adjoint form of any connected semisimple real Lie group. (In the complex case, the notions of connectivity in the ordinary (Hausdorff) topology and the Zariski topology coincide).

Let $G$ be a connected semisimple $\mathbb{R}$-group.

A subgroup $P \subset G$ is called parabolic, if it contains a Borel subgroup, i. e. a maximal solvable connected (in fact, the connectivity follows) 
subgroup. In this case, $G / P$ is compact and a projective algebraic variety (i. e. a subset of $\mathbb{C P}^{n}$ defined by homogenous polynomials).

We assume that $G(\mathbb{R})$ is non-compact (otherwise the following would be trivial). Let $S$ be a maximal $\mathbb{R}$-split torus, i.e. a maximal algebraic torus $S$, such that the adjoint action of $S(\mathbb{R})$ on $\mathfrak{g}$ has only real eigenvalues. The dimension $r$ of $S$ is called $\mathbb{R}$-rank of $G$ (this is well-defined, since all maximal $\mathbb{R}$-split tori are conjugate, [Bo, 20.9]). Then $\mathfrak{g}$ is the sum of relative root spaces $\mathfrak{g}=\bigoplus_{\alpha \in \Phi_{\mathbb{R}}} \mathfrak{g}_{\alpha}$ (in general not one-dimensional), where $\Phi_{\mathbb{R}} \subset \mathfrak{s}^{*}$ is the relative root system. The group generated by the orthogonal reflections through the hyperplanes $\left\{\alpha^{\perp}: \alpha \in \Phi_{\mathbb{R}}\right\}$ is the relative Weyl group $\mathcal{W}_{\mathbb{R}}=\mathcal{N}(S) / \mathcal{Z}(S)$, which can be represented by elements of $G(\mathbb{R})[\mathrm{Bo}, 21.2]$. The connected components of $\mathfrak{s} \backslash \bigcup_{\alpha \in \Phi_{\mathbb{R}}} \alpha^{\perp}$ are called relative Weyl chambers. The choice of a relative Weyl chamber determines a partition $\Phi_{\mathbb{R}}=\Phi_{\mathbb{R}}^{+} \cup \Phi_{\mathbb{R}}^{-}$of the set of roots into positive and negative ones. Let $\Delta_{\mathbb{R}} \subset \Phi_{\mathbb{R}}^{+}$denote the set of simple roots, i. e. the positive roots that are not the sum of two other positive roots. Clearly, the simple roots $\left(\alpha_{1}, \ldots, \alpha_{r}\right), r=\operatorname{rank}_{\mathbb{R}}(G)$ form a basis of $\mathfrak{s}^{*}$. Let $\left(\xi_{1}, \ldots, \xi_{r}\right)$ be the basis of $\mathfrak{s}$ dual to this, i. e. $\alpha_{a}\left(\xi_{b}\right)=\delta_{a b}$.

The following proposition relates subsets of $\Delta_{\mathbb{R}}$ to semi-canonical elements and to parabolic $\mathbb{R}$-subgroups (cf. [Bo, 21.12]):

Proposition 3.3.11. There is a bijection between:

- subsets of $\Delta_{\mathbb{R}}$,

- conjugacy classes of semi-canonical elements and

- conjugacy classes of parabolic $\mathbb{R}$-subgroups.

More explicitly:

(1) For each subset $I \subset \Delta_{\mathbb{R}}$, the element $\xi_{I}:=\sum_{\alpha_{a} \in \Delta_{\mathbb{R}} \backslash I} \xi_{a}$ is semi-canonical.

(2) Each semi-canonical element is $G(\mathbb{R})$-conjugate to exactly one of the $\xi_{I}$.

(3) If $\xi$ is semi-canonical, then the connected $\mathbb{R}$-subgroup $P_{\xi}=\left\{g \in G: \operatorname{Ad}_{g} \xi \in \xi+\bigoplus_{\alpha(\xi)>0} \mathfrak{g}_{\alpha}\right\}$ with Lie algebra $\mathfrak{p}_{\xi}=\bigoplus_{\alpha(\xi) \geq 0} \mathfrak{g}_{\alpha}$ is parabolic.

(4) For each parabolic $\mathbb{R}$-subgroup $P$ there exists a unique semicanonical element $\xi$, such that $P=P_{\xi}$.

Proof. (1) By definition, the $\operatorname{ad}_{\xi}$-eigenspaces are $\mathfrak{g}_{\lambda}=\bigoplus_{\alpha(\xi)=\lambda} \mathfrak{g}_{\alpha}$. Let $\xi$ be as described. Then we have $\alpha(\xi) \in\{0,1\}$ for each simple root $\alpha$. Since each positive root is the sum of simple ones, it follows that $\alpha(\xi) \in \mathbb{N}_{0}$ for each positive root $\alpha$ (and of course $-\alpha(\xi) \in-\mathbb{N}_{0}$ for each negative root $-\alpha$ ). Because $\mathfrak{g}_{0} \oplus \mathfrak{g}_{1}$ contains all simple root spaces, it 
generates the subalgebra $\bigoplus_{\lambda \geq 0} \mathfrak{g}_{\lambda}$. From $\left[\mathfrak{g}_{0}, \mathfrak{g}_{1}\right] \subset \mathfrak{g}_{1}$ it then follows that $\mathfrak{g}_{1}$ generates $\bigoplus_{\lambda>0} \mathfrak{g}_{\lambda}$. Analogously for $\mathfrak{g}_{-1}$.

(3) By definition, $\xi$ is semisimple, so it is contained in a maximal torus $\mathfrak{t} \subset \mathfrak{g}_{0}$. Choose a Weyl chamber in $\mathfrak{t}^{*}$ whose closure contains $\xi$. This defines a positive direction in $\mathfrak{t}$, such that $\mathfrak{p}_{\xi}$ contains the sum of $\mathfrak{t}$ and all positive root spaces. The latter is easily seen to be a Borel subalgebra, therefore $\mathfrak{p}_{\xi}$ is parabolic.

Now (2) and (4) follow from the fact that each parabolic $\mathbb{R}$-group is $G(\mathbb{R})$-conjugate to exactly one of the $P_{\xi_{I}}[\mathrm{BO}, 21.12]$, and that $P_{\xi}=$ $P_{\xi^{\prime}}$ only if $\xi=\xi^{\prime}$ : This follows from the observation that the $\mathrm{ad}_{\xi^{-}}$ eigenspaces $\mathfrak{g}_{\lambda}$ (and therefore $\xi$, by semisimplicity) are determined by $\mathfrak{p}_{\xi}$ and $\mathfrak{p}_{-\xi}$ : Let $\mathfrak{n}_{ \pm}$be the nilradical of $\mathfrak{p}_{ \pm \xi}$. Then $\mathfrak{D}_{a} \mathfrak{n}_{ \pm}=\bigoplus_{\lambda>a+1} \mathfrak{g}_{ \pm \lambda}$ (where $\mathfrak{D}_{0} \mathfrak{n}:=\mathfrak{n}, \mathfrak{D}_{a+1} \mathfrak{n}:=\left[\mathfrak{n}, \mathfrak{D}_{a} \mathfrak{n}\right]$ ). Because $\mathfrak{g}_{\lambda} \perp \mathfrak{g}_{\mu}$ for $\bar{\lambda} \neq-\mu$ with respect to the Killing form, it follows that $\mathfrak{g}_{0}=\mathfrak{p}_{+} \cap \mathfrak{n}_{-}^{\perp}$ and $\mathfrak{g}_{ \pm a}=\mathfrak{D}_{a-1} \mathfrak{n}_{ \pm} \cap\left(\mathfrak{D}_{a} \mathfrak{n}_{\mp}\right)^{\perp}$ for $a \in \mathbb{N}$.

There are two special cases of this construction: For $I=\Delta_{\mathbb{R}}$ we obtain $\xi=0$ and $P_{\Delta_{\mathbb{R}}}=G$. - For $I=\emptyset$ we get the minimal parabolic $\mathbb{R}$ subgroups, which play the same role in the real Bruhat decomposition as the Borel subgroups in the complex case:

TheOREM. [Bo, 21.15, 21.16] Let $G$ be a connected semisimple $\mathbb{R}$ group, $S$ a maximal $\mathbb{R}$-split torus and $P_{\emptyset}$ a minimal parabolic $\mathbb{R}$-subgroup. Then

$$
G(\mathbb{R})=\bigcup_{w \in \mathcal{W}_{\mathbb{R}}} P_{\emptyset}(\mathbb{R}) w P_{\emptyset}(\mathbb{R}) \quad \text { (disjoint union). }
$$

More generally, let $P_{I} \supset P_{\emptyset}$ be a parabolic $\mathbb{R}$-subgroup of $G$ and let $\mathcal{W}_{I} \subset \mathcal{W}_{\mathbb{R}}$ be the subgroup generated by the reflections through the $\left\{\alpha^{\perp}: \alpha \in I\right\}$, then

$$
G(\mathbb{R})=\bigcup_{w \in \mathcal{W}_{\mathbb{R}} / \mathcal{W}_{I}} P_{\emptyset}(\mathbb{R}) w P_{I}(\mathbb{R}) .
$$

Therefore, $G(\mathbb{R}) / P_{I}(\mathbb{R})$ is a union of the Bruhat cells

$$
G(\mathbb{R}) / P_{I}(\mathbb{R})=\bigcup_{w \in \mathcal{W}_{\mathbb{R}} / \mathcal{W}_{I}} P_{\emptyset}(\mathbb{R}) w P_{I}(\mathbb{R}) / P_{I}(\mathbb{R})
$$

Let $w$ be the element of shortest length in its class $w \cdot \mathcal{W}_{I}$; then the cell $P_{\emptyset} w P_{I} / P_{I}$ is $\mathbb{R}$-isomorphic to the affine space $N_{w}:=N_{\emptyset} \cap w N_{\emptyset} w^{-1}$, where $N_{\emptyset}, N_{\emptyset}$ are the unipotent radicals of $P_{\emptyset}$ and of its opposite parabolic subgroup $P_{\emptyset}:=P_{-\xi_{\emptyset}}$, respectively [Bo, 21.20, 21.29]. Note that $\exp : \mathfrak{n}_{w} \mapsto N_{w}$ is an $\mathbb{R}$-isomorphism, because $\mathfrak{n}_{w}$ is nilpotent and the exponential series and its inverse are finite.

There is one big cell in this decomposition, belonging to the element $w_{0}$ which maps $\Delta_{\mathbb{R}} \backslash I$ to $\Phi_{\mathbb{R}}^{-}$: The image of $N_{w_{0}} \cong N_{I-}$ is $\mathbb{R}$-Zariski-open 
and -dense in $G / P_{I}$. Moreover, the map $N_{I-} \rightarrow G / P_{I}$ is a birational equivalence (meaning that it induces an isomorphism of the ring of rational functions $\left.\mathbb{C}\left(G / P_{I}\right) \stackrel{\sim}{\rightarrow} \mathbb{C}\left(N_{I-}\right)\right)$ [Bo, 21.20].

Summarizing this we conclude (back to our original notation, where the real groups are denoted $G, P_{ \pm}, \ldots$ rather than $G(\mathbb{R}), P_{ \pm}(\mathbb{R}), \ldots$ ):

THEOREM 3.3.12. Let $G$ be a noncompact real form of a connected semisimple linear algebraic group. Let $\xi \in \mathfrak{g}$ be semi-canonical and $G^{\mathbf{c}} / P_{-}^{\mathbf{c}}$ the closed twistor space associated to $\xi$. Then the following facts hold:

(1) The quotient $G^{\mathbf{c}} / P_{-}^{\mathbf{c}}$ is a product $\left(G / P_{-}\right) \times\left(G / P_{+}\right)$of real projective varieties.

(2) $\left(G / P_{-}\right) \times\left(G / P_{+}\right)$is a disjoint union of affine spaces, namely the products of the Bruhat cells

$$
\bigcup_{\left(w_{+}, w_{-}\right) \in \mathcal{W} \times \mathcal{W}}\left(P_{\emptyset} w_{+} P_{-} / P_{-}\right) \times\left(P_{\emptyset} w_{-} P_{+} / P_{+}\right)
$$

(3) The map $\mathfrak{n}_{+} \times \mathfrak{n}_{-} \stackrel{\exp }{\longrightarrow} N_{+} \times N_{-} \rightarrow\left(G / P_{-}\right) \times\left(G / P_{+}\right)$is an embedding onto a Zariski-open and-dense subset (namely the product of the respective big Bruhat cells) and induces a birational equivalence $\mathfrak{n}_{+} \times \mathfrak{n}_{-} \sim\left(G / P_{-}\right) \times\left(G / P_{+}\right)$.

3.3.3. Twistor fibrations of $\mathbb{R}$-split simple Lie groups. By Proposition 3.3.4, in order to find the twistor fibrations of a given Lie group $G$ and therefore the isotropic para-pluriharmonic maps into $G$-symmetric spaces (Theorem 3.3.6), we have to find the canonical elements in $\mathfrak{g}$. A direct consequence of Proposition 3.3.11 is the following

COROLlary 3.3.13. Let $G$ be a simple Lie group of $\mathbb{R}-r a n k r$. Then there exist - up to covering or isomorphism - $2^{r}-1$ nontrivial G-twistor fibrations, each of which corresponds to a proper subset of $\Delta_{\mathbb{R}}$.

This construction is fairly straightforward, if $G$ has an $\mathbb{R}$-split maximal torus (i. e. one that is maximal among all tori). These groups are called $\mathbb{R}$-split.

As an example, we will carry this out for $\operatorname{Sp}(2 n, \mathbb{R})$ :

The group $\operatorname{Sp}(2 n, \mathbb{R})$ is defined as the group of automorphisms of $\mathbb{R}^{2 n}$ which preserve the standard symplectic form given by $\Omega_{2 n}=$ $\left(\begin{array}{cc}0 & -\mathbf{1}_{n} \\ \mathbf{1}_{n} & 0\end{array}\right)$. Its Lie algebra is

$$
\mathfrak{s p}(2 n, \mathbb{R})=\left\{\left(\begin{array}{cc}
A & C \\
B & -A^{t}
\end{array}\right): A, B, C \in \mathfrak{g l}(n, \mathbb{R}), B, C \text { symmetric }\right\} .
$$


The group $\operatorname{Sp}(2 n, \mathbb{R})$ has $\mathbb{R}$-rank $n$, and a system of simple coroots spanning the maximal torus is given by

$$
\begin{aligned}
& \xi_{a}:=\left(\begin{array}{cc}
D_{a} & \\
& -D_{a}
\end{array}\right), D_{a}:=\left(\begin{array}{cc}
\mathbf{0}_{a} & \\
& \mathbf{1}_{n-a}
\end{array}\right) \text { for } 1 \leq a \leq n-1 \text { and } \\
& \xi_{n}:=\left(\begin{array}{cc}
\frac{1}{2} \mathbf{1}_{n} & \\
& -\frac{1}{2} \mathbf{1}_{n}
\end{array}\right)
\end{aligned}
$$

( $\alpha_{n}$ is the long root of the Dynkin diagram $\underset{\alpha_{1}}{\circ-} \circ \cdots-0<0$ - $\alpha_{n}$ ).

There are two types of canonical elements:

Case 1: $\alpha_{n} \in I$, i. e. $\xi$ does not contain the summand $\xi_{n}$. In this case we have

$$
\xi=\left(\begin{array}{cc}
D & 0 \\
0 & -D
\end{array}\right), D=\left(\begin{array}{cccc}
0 \cdot \mathbf{1}_{m_{1}} & & & \\
& 1 \cdot \mathbf{1}_{m_{2}} & & \\
& & 2 \cdot \mathbf{1}_{m_{3}} & \\
& & & \ddots
\end{array}\right)
$$

The centralizer of $\xi$ is

$$
H=\mathrm{Sp}\left(2 m_{1}, \mathbb{R}\right) \times \mathrm{GL}\left(m_{2}, \mathbb{R}\right) \times \mathrm{GL}\left(m_{3}, \mathbb{R}\right) \times \cdots
$$

$\left(\right.$ where $\left.\operatorname{GL}(n, \mathbb{R})=\left\{\left(\begin{array}{cc}A & 0 \\ 0 & \left(A^{-1}\right)^{t}\end{array}\right): A \in \mathrm{GL}(n, \mathbb{R})\right\} \subset \mathrm{Sp}(2 n, \mathbb{R})\right)$, and the centralizer of $e^{i \pi \xi}$ is

$$
G^{\sigma}=\operatorname{Sp}\left(2 m_{\text {odd }}, \mathbb{R}\right) \times \operatorname{Sp}\left(2 m_{\mathrm{ev}}, \mathbb{R}\right),
$$

where $m_{\mathrm{odd}}:=\sum_{a \text { odd }} m_{a}, m_{\mathrm{ev}}:=\sum_{a \text { even }} m_{a}$.

The resulting twistor fibration $G / H \rightarrow G / K\left(\right.$ with $K:=H \cdot G_{0}^{\sigma}=G^{\sigma}$ ) is

$$
\begin{gathered}
\operatorname{Sp}(2 n, \mathbb{R}) / \operatorname{Sp}\left(2 m_{1}, \mathbb{R}\right) \times \operatorname{GL}\left(m_{2}, \mathbb{R}\right) \times \operatorname{GL}\left(m_{3}, \mathbb{R}\right) \times \cdots \\
\downarrow \\
\operatorname{Sp}(2 n, \mathbb{R}) / \operatorname{Sp}\left(2 m_{\text {ev }}, \mathbb{R}\right) \times \operatorname{Sp}\left(2 m_{\text {odd }}, \mathbb{R}\right) .
\end{gathered}
$$

Case 2: $\alpha_{n} \notin I$, i. e. $\xi$ contains the summand $\xi_{n}$. In this case we have

$$
\xi=\left(\begin{array}{cc}
D & 0 \\
0 & -D
\end{array}\right), D=\left(\begin{array}{cccc}
\frac{1}{2} \mathbf{1}_{m_{1}} & & & \\
& \frac{3}{2} \mathbf{1}_{m_{2}} & & \\
& & \frac{5}{2} \mathbf{1}_{m_{3}} & \\
& & & \ddots
\end{array}\right)
$$

In order to compute the twistor fibration, it is practical to switch to a different Weyl chamber and change $\xi$ into

$$
\xi^{\prime}=\left(\begin{array}{cc}
D^{\prime} & 0 \\
0 & -D^{\prime}
\end{array}\right), D^{\prime}=\left(\begin{array}{cccc}
\frac{1}{2} \mathbf{1}_{m_{1}} & & & \\
& -\frac{3}{2} \mathbf{1}_{m_{2}} & & \\
& & \frac{5}{2} \mathbf{1}_{m_{3}} & \\
& & & \mp \cdot \cdot
\end{array}\right)
$$


Then the corresponding twistor fibration $G / H \rightarrow G / K$ (with $K=$ $\left.H \cdot G_{0}^{\sigma}=G^{\sigma}\right)$ is

$$
\begin{gathered}
\mathrm{Sp}(2 n, \mathbb{R}) / \mathrm{GL}\left(m_{1}, \mathbb{R}\right) \times \mathrm{GL}\left(m_{2}, \mathbb{R}\right) \times \cdots \\
\qquad \operatorname{Sp}(2 n, \mathbb{R}) / \mathrm{GL}(n, \mathbb{R}) .
\end{gathered}
$$

(The original $\xi$ would lead to a subgroup conjugate to this.)

Note that $\operatorname{Sp}(2 n, \mathbb{R}) / \mathrm{GL}(n, \mathbb{R})$ is the adjoint orbit $\operatorname{Ad}_{G}\left(\xi_{n}\right)$ and therefore has a para-complex structure. However, the map $\operatorname{Ad}_{\operatorname{Sp}(2 n, \mathbb{R})}\left(\xi^{\prime}\right) \rightarrow$ $\operatorname{Ad}_{\operatorname{Sp}(2 n, \mathbb{R})}\left(\xi_{n}\right)$ is not holomorphic, except in the trivial case $\xi^{\prime}=\xi_{n}$.

In the same way we can compute the twistor fibrations $G / H \rightarrow G /(H$. $\left.G_{0}^{\sigma}\right)$ of all classical $\mathbb{R}$-split simple Lie groups:

\begin{tabular}{|c|c|c|c|}
\hline $\mathrm{A}_{n}$ & $\begin{array}{l}\mathrm{SL}(n, \mathbb{R}) \\
\stackrel{\mathrm{O}}{\mathrm{\alpha}} \mathrm{O} \cdot \cdots \mathrm{\circ}-\underset{\alpha_{n}}{\mathrm{O}}\end{array}$ & & $\begin{array}{c}\mathrm{SL}(n, \mathbb{R}) / \mathrm{S}\left(\mathrm{GL}\left(m_{1}, \mathbb{R}\right) \times \mathrm{GL}\left(m_{2}, \mathbb{R}\right) \cdots\right) \\
\downarrow \\
\mathrm{SL}(n, \mathbb{R}) / \mathrm{S}\left(\mathrm{GL}\left(m_{\text {odd }}, \mathbb{R}\right) \times \mathrm{GL}\left(m_{\text {ev }}, \mathbb{R}\right)\right)\end{array}$ \\
\hline \multirow[t]{2}{*}{$\mathrm{B}_{n}$} & $\mathrm{SO}(n+1, n)$ & $\alpha_{n} \notin I$ & $\begin{array}{c}\mathrm{SO}(n+1, n) / \mathrm{GL}\left(m_{1}, \mathbb{R}\right) \times \mathrm{GL}\left(m_{2}, \mathbb{R}\right) \times \mathrm{GL}\left(m_{3}, \mathbb{R}\right) \times \cdots \\
\downarrow \\
\mathrm{SO}(n+1, n) / \mathrm{SO}\left(m_{\text {odd }}, m_{\text {odd }}\right) \times \mathrm{SO}\left(m_{\mathrm{ev}}+1, m_{\mathrm{ev}}\right)\end{array}$ \\
\hline & $\mathrm{O}_{1} \cdots \stackrel{\circ}{\mathrm{\alpha}} \underset{\alpha_{n-1}}{\Rightarrow} \alpha_{n}$ & $\alpha_{n} \in I$ & $\begin{array}{c}\mathrm{SO}(n+1, n) / \mathrm{SO}\left(m_{1}+1, m_{1}\right) \times \mathrm{GL}\left(m_{2}, \mathbb{R}\right) \times \mathrm{GL}\left(m_{3}, \mathbb{R}\right) \times \cdots \\
\downarrow \\
\mathrm{SO}(n+1, n) / \mathrm{SO}\left(m_{\text {odd }}+1, m_{\text {odd }}\right) \times \mathrm{SO}\left(m_{\text {ev }}, m_{\text {ev }}\right)\end{array}$ \\
\hline \multirow[t]{2}{*}{$\mathrm{C}_{n}$} & $\operatorname{Sp}(2 n, \mathbb{R})$ & $\alpha_{n} \notin I$ & 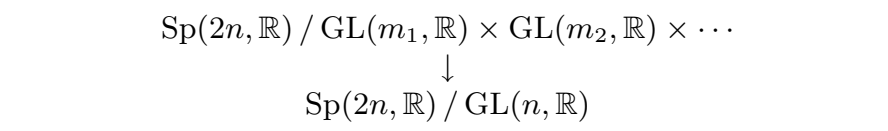 \\
\hline & $\stackrel{\circ}{\circ} \cdot \cdots \underset{\alpha_{1}}{\circ} \underset{\alpha_{n-1}}{\circ}<\alpha_{n}$ & $\alpha_{n} \in I$ & $\begin{array}{c}\mathrm{Sp}(2 n, \mathbb{R}) / \operatorname{Sp}\left(2 m_{1}, \mathbb{R}\right) \times \operatorname{GL}\left(m_{2}, \mathbb{R}\right) \times \mathrm{GL}\left(m_{3}, \mathbb{R}\right) \times \cdots \\
\downarrow \\
\operatorname{Sp}(2 n, \mathbb{R}) / \operatorname{Sp}\left(2 m_{\text {odd }}, \mathbb{R}\right) \times \operatorname{Sp}\left(2 m_{\mathrm{ev}}, \mathbb{R}\right)\end{array}$ \\
\hline \multirow[t]{2}{*}{$\mathrm{D}_{n}$} & $\mathrm{SO}(n, n)$ & $\begin{array}{l}\text { either } \\
\alpha_{n} \in I \\
\quad \text { or } \\
\alpha_{n-1} \in I\end{array}$ & $\begin{array}{c}\mathrm{SO}(n, n) / \mathrm{GL}\left(m_{1}, \mathbb{R}\right) \times \mathrm{GL}\left(m_{2}, \mathbb{R}\right) \times \mathrm{GL}\left(m_{3}, \mathbb{R}\right) \times \cdots \\
\downarrow \\
\operatorname{SO}(n, n) / \mathrm{GL}(n, \mathbb{R})\end{array}$ \\
\hline & $\mathrm{O}_{\alpha_{1}}^{\circ} \cdots \mathrm{O}_{\alpha_{n}}^{\mathrm{o}_{n-1}}$ & $\begin{array}{l}\text { both } \\
\text { or } \\
\text { neither }\end{array}$ & $\begin{array}{c}\mathrm{SO}(n, n) / \mathrm{SO}\left(m_{1}, m_{1}\right) \times \mathrm{GL}\left(m_{2}, \mathbb{R}\right) \times \mathrm{GL}\left(m_{3}, \mathbb{R}\right) \times \cdots \\
\downarrow \\
\mathrm{SO}(n, n) / \mathrm{SO}\left(m_{\text {odd }}, m_{\text {odd }}\right) \times \mathrm{SO}\left(m_{\text {ev }}, m_{\text {ev }}\right)\end{array}$ \\
\hline
\end{tabular}

TABLE 1. Twistor fibrations of classical $\mathbb{R}$-split simple groups

REMARK. The reader may have observed that $\mathrm{SO}(n, n)$ and $\mathrm{SO}(n+1, n)$ are not connected. However, the only place where this assumption was needed, 
was the proof of $\sigma(G)=G$ (Proposition 3.3.4). Since this holds nonetheless, we stick with these groups rather than use their (non-algebraic) identity components.

\subsection{Isotropic para-pluriharmonic maps into Grassmannians}

The fibrations in the first row of this table are in fact the fibrations of para-complex flag manifolds over para-complex Grassmannians, which we will now describe more in detail, giving an explicit construction of para-pluriharmonic maps.

Definition 3.4.1. Let $m_{1}, \ldots, m_{l} \in \mathbb{N}$ and $n:=\sum m_{a}$. The paracomplex flag manifold $\mathrm{F}_{m_{1}, \ldots, m_{l}}\left(\mathbf{C}^{n}\right)$ is the space of nondegenerate $\left(m_{1}, \ldots, m_{l}\right)$-flags in $\mathbf{C}^{n}$; such a flag is an orthogonal decomposition of $\mathbf{C}^{n}$ into nondegenerate (with respect to the standard para-hermitian scalar product) subspaces $\mathbf{C}^{n}=E_{1} \oplus \cdots \oplus E_{l}, \operatorname{dim}_{\mathbf{C}}\left(E_{a}\right)=m_{a}$. Equivalently, a flag is a sequence of nondegenerate subspaces $\{0\}=$ $W_{0} \subsetneq W_{1} \subsetneq \cdots \subsetneq W_{l}=\mathbf{C}^{n}, \operatorname{dim}\left(W_{a}\right)=m_{1}+\cdots+m_{a}$, where $W_{a}:=E_{1} \oplus \cdots \oplus E_{a}$. (To see the equivalence, we use the fact that the orthogonal complement of a nondegenerate subspace is again nondegenerate.)

The flag manifolds $\mathrm{F}_{m, n-m}\left(\mathbf{C}^{n}\right)=: \mathrm{Gr}_{m}\left(\mathbf{C}^{n}\right)$ are called para-complex Grassmannians.

The standard action of $\mathrm{U}^{\pi}(n)$ on $\mathbf{C}^{n}$ induces a transitive (by Proposition 1.3.3) $\mathrm{U}^{\pi}(n)$-action on $\mathrm{F}_{m_{1}, \ldots, m_{l}}\left(\mathbf{C}^{n}\right)$. There is an equivariant projection map $\mathrm{U}^{\pi}(n) \rightarrow \mathrm{F}_{m_{1}, \ldots, m_{l}}\left(\mathbf{C}^{n}\right)$ which sends a matrix $g \in \mathrm{U}^{\pi}(n)$ to the flag $\left(E_{1}, \ldots, E_{l}\right)$, where $E_{1}$ is the span of the first $m_{1}$ columns of $g, E_{2}$ the span of the next $m_{2}$ columns, and so on. In particular, the identity matrix is mapped to the standard flag $\mathbf{C}^{n}=\mathbf{C}^{m_{1}} \oplus \cdots \oplus \mathbf{C}^{m_{l}}$. The restriction of this map to $\mathrm{SU}^{\pi}(n)$ is still surjective, since we can multiply the first column of a given matrix $g \in \mathrm{U}^{\pi}(n)$ by $\operatorname{det}(g)^{-1} \in$ $\{z \in \mathbf{C}: \bar{z} z=1\}$ without changing its span.

This yields the identification

$$
\mathrm{F}_{\left(m_{1}, \ldots, m_{l}\right)}\left(\mathbf{C}^{n}\right) \cong \mathrm{SU}^{\pi}(n) / \mathrm{S}\left(\mathrm{U}^{\pi}\left(m_{1}\right) \times \cdots \times \mathrm{U}^{\pi}\left(m_{l}\right)\right) .
$$

Now recall that $\mathrm{SU}^{\pi}(n) \cong \operatorname{SL}(n, \mathbb{R})$. So the fibration

$$
\begin{gathered}
\operatorname{SL}(n, \mathbb{R}) / \operatorname{S}\left(\operatorname{GL}\left(m_{1}, \mathbb{R}\right) \times \cdots \times \operatorname{GL}\left(m_{l}, \mathbb{R}\right)\right) \\
\downarrow \\
\operatorname{SL}(n, \mathbb{R}) / \operatorname{S}\left(\operatorname{GL}\left(m_{\text {odd }}, \mathbb{R}\right) \times \operatorname{GL}\left(m_{\text {ev }}, \mathbb{R}\right)\right)
\end{gathered}
$$

(cf. Table 1) is the map $\mathrm{F}_{\left(m_{1}, \ldots, m_{l}\right)}\left(\mathbf{C}^{n}\right) \rightarrow \operatorname{Gr}_{m_{\text {odd }}}\left(\mathbf{C}^{n}\right)$, which sends the flag $\left(E_{1}, \ldots, E_{l}\right)$ to the subspace $\bigoplus_{a \text { odd }} E_{a} \subset \mathbf{C}^{n}$. The corresponding 
canonical element is

$$
\xi=\left(\begin{array}{ccc}
0 \cdot \mathbf{1}_{m_{1}} & & \\
& \ddots & \\
& & (l-1) \mathbf{1}_{m_{l}}
\end{array}\right)+\lambda \mathbf{1}_{n} \in \mathfrak{s l}(n, \mathbb{R})
$$

(where $\lambda$ is the unique real number, such that $\xi$ is trace free). By the identification $\mathfrak{s l}(n, \mathbb{R}) \stackrel{\sim}{\rightarrow} \mathfrak{s u}^{\pi}(n), X \mapsto \frac{1+j}{2} X-\frac{1-j}{2} X^{t}$, this corresponds to

$$
\xi=\left(\begin{array}{ccc}
0 \cdot j \cdot \mathbf{1}_{m_{1}} & & \\
& \ddots & \\
& & (l-1) j \cdot \mathbf{1}_{m_{l}}
\end{array}\right)+\lambda j \mathbf{1}_{n} \in \mathfrak{u}^{\pi}(n) .
$$

LEMMA.

$$
\begin{aligned}
& T_{\left(E_{1}, \ldots, E_{l}\right)} Z=\left\{\left(X_{a b}\right) \in \bigoplus_{a \neq b} \operatorname{Hom}\left(E_{a}, E_{b}\right): X_{a b}+X_{b a}^{*}=0\right\} \\
& T_{\left(E_{1}, \ldots, E_{l}\right)}^{\mathbf{c}} Z=\bigoplus_{a \neq b} \operatorname{Hom}\left(E_{a}, E_{b}\right)
\end{aligned}
$$

ProOF. The second equation follows immediately from the first. So let $\pi_{a}$ denote the orthogonal projection on $E_{a}$. From $\pi_{a}=\pi_{a}^{*}, \pi_{a}^{2}=\pi_{a}$ and $\pi_{a} \pi_{b}=0, a \neq b$ it follows that a tangent vector to $Z$ at $E=\left(E_{a}\right)$ is given by a collection of maps $\pi_{1}^{\prime}, \ldots \pi_{l}^{\prime}: \mathbf{C}^{n} \rightarrow \mathbf{C}^{n}$ satisfying

$$
\pi_{a}^{\prime}=\pi_{a}^{\prime *}, \quad \pi_{a}^{\prime} \pi_{a}+\pi_{a} \pi_{a}^{\prime}=\pi_{a}^{\prime}, \quad \pi_{a}^{\prime} \pi_{b}+\pi_{a} \pi_{b}^{\prime}=0 \quad \text { for } a \neq b .
$$

Now it is easy to see that that $X_{a b}:=\pi_{b} \pi_{a}^{\prime}=-\pi_{b}^{\prime} \pi_{a} \in \operatorname{Hom}\left(E_{a}, E_{b}\right)$ for $a \neq b$ satisfies $X_{a b}+X_{b a}^{*}=0$ and that any such collection $\left(X_{a b}\right)$ defines maps $\pi_{a}^{\prime}$ with the above properties.

By definition, $\operatorname{ad}_{\xi}$ has eigenvalue $(b-a) j$ on $\operatorname{Hom}\left(\mathbf{C}^{m_{a}}, \mathbf{C}^{m_{b}}\right)$, therefore the superhorizontal bundle is $\operatorname{Ad}_{g}\left(\mathfrak{g}_{1}^{\mathbf{c}}\right)=\bigoplus_{a} \operatorname{Hom}\left(E_{a}, E_{a+1}\right) \subset T_{\left(E_{a}\right)}^{\mathbf{c}} Z$. A holomorphic map $f: N \rightarrow Z$ from a para-Kähler manifold $N$ to $Z=\mathrm{F}_{\left(m_{1}, \ldots, m_{l}\right)}\left(\mathbf{C}^{n}\right)$ is superhorizontal, if and only if $\partial f=d f^{(1,0)}$ takes values in $\bigoplus_{a} \operatorname{Hom}\left(E_{a}, E_{a+1}\right)$, or, equivalently (as follows by induction), if $\partial f$ maps $W_{a}=E_{1} \oplus \cdots \oplus E_{a}$ to $W_{a+1}$. Thus we can construct parapluriharmonic maps in the following way: Let $f_{1}, \ldots, f_{m_{1}}: N \rightarrow \mathbf{C}^{n}$ be holomorphic maps such that for $n \in N$ the images $f_{a}(n)$ are C-linearly independent vectors with nondegenerate span. Let $W_{1}$ be the span of the $f_{a}, W_{2}$ the span of the $f_{a}$ and their derivatives and possibly further $g_{1}, g_{2}, \ldots: N \rightarrow \mathbf{C}^{n}$ and so on, as long as all these maps are (pointwise) linearly independent and span nondegenerate subspaces. This defines a superhorizontal map $F: N \rightarrow \mathrm{F}_{\left(m_{1}, \ldots, m_{l}\right)}\left(\mathbf{C}^{n}\right)$, and the composition $f=\pi \circ F: N \rightarrow \mathrm{Gr}_{m_{\text {odd }}}\left(\mathbf{C}^{n}\right)$ is para-pluriharmonic.

A special case of this is the fibration $\mathrm{F}_{(1, n, 1)}\left(\mathbf{C}^{n+2}\right) \rightarrow \operatorname{Gr}_{2}\left(\mathbf{C}^{n+2}\right)$, which is the twistor fibration associated to the para-quaternionic Kähler structure on $\operatorname{Gr}_{2}\left(\mathbf{C}^{n+2}\right)$ (cf. section 3.6.1). Para-pluriharmonic 
maps $f: N \rightarrow \operatorname{Gr}_{2}\left(\mathbf{C}^{n+2}\right), \operatorname{dim}_{\mathbf{C}} N=n$, are obtained by (para-)holomorphic functions $h: N \rightarrow \mathbf{C}^{n+2}$ via $f=W_{1} \oplus W_{2}^{\perp}$, where $W_{1}(n)=$ $\mathbf{C} \cdot h(n)$ and $W_{2}(n)=\operatorname{span}_{\mathbf{C}}(h(n), \operatorname{grad}(h(n)))$. In the next section we will prove that, given two para-quaternionic Kähler symmetric spaces of the same dimension, there is a biholomorphism between open and dense subsets (the big Bruhat cells, see above) of their twistor spaces preserving the horizontal structure. This can be used to transfer the preceding construction to any para-quaternionic Kähler symmetric space.

\subsection{Para-quaternionic Kähler symmetric spaces}

In this section, we examine the twistor fibrations $Z \rightarrow M$ associated to a para-quaternionic Kähler structure on $M$. We will see that these are the twistor spaces which belong to a depth-two grading $\mathfrak{g}=\mathfrak{g}_{-2} \oplus$ $\mathfrak{g}_{-1} \oplus \mathfrak{g}_{0} \oplus \mathfrak{g}_{1} \oplus \mathfrak{g}_{2}$ with $\operatorname{dim}\left(\mathfrak{g}_{ \pm 2}\right)=1$. The main result is the birational equivalence between all these twistor spaces of the same dimension.

Proposition 3.5.1. Let $M=G / K$ be a pseudo-Riemannian symmetric space such that $G$ acts effectively on $M$.

(1) If $M$ is quaternionic Kähler with nonzero scalar curvature (see definition 1.4.4), then there exists a semi-canonical element $\xi \in \mathfrak{k}$ which defines a grading $\mathfrak{g}=\mathfrak{g}_{-2} \oplus \mathfrak{g}_{-1} \oplus \mathfrak{g}_{0} \oplus \mathfrak{g}_{1} \oplus \mathfrak{g}_{2}$ such that $\xi \in\left[\mathfrak{g}_{-2}, \mathfrak{g}_{2}\right], \mathfrak{g}_{-2} \oplus \mathfrak{g}_{0} \oplus \mathfrak{g}_{2}=\mathfrak{k}, \mathfrak{g}_{-1} \oplus \mathfrak{g}_{1}=\mathfrak{m}, \operatorname{dim}\left(\mathfrak{g}_{-2}\right)=$ $\operatorname{dim}\left(\mathfrak{g}_{2}\right)=1$ and $[]:, \mathfrak{g}_{ \pm 1} \times \mathfrak{g}_{ \pm 1} \rightarrow \mathfrak{g}_{ \pm 2}$ is nondegenerate.

(2) Vice versa, if there exist a semi-canonical $\xi \in \mathfrak{k}$ which defines a grading $\mathfrak{g}=\mathfrak{g}_{-2} \oplus \mathfrak{g}_{-1} \oplus \mathfrak{g}_{0} \oplus \mathfrak{g}_{1} \oplus \mathfrak{g}_{2}$ as above and if $K \subset$ $H \cdot G_{0}^{\sigma}$ (where $H$ is the centralizer of $\xi$ in $G$ ), then $M$ has a $G$-invariant para-quaternionic Kähler structure.

(3) If $K=H \cdot G_{0}^{\sigma}$, the twistor space $\operatorname{Ad}_{G}(\xi)=G / H$ is the twistor space of para-complex structures (Definition 2.2.1).

REMARK. Of course, there may be other twistor bundles over the same base space $M$. A simple example is the trivial fibration $\operatorname{Gr}_{2}\left(\mathbf{C}^{n+2}\right) \rightarrow$ $\mathrm{Gr}_{2}\left(\mathbf{C}^{n+2}\right)$.

Proof. (1): Suppose $M$ is a para-quaternionic Kähler manifold. Then $\mathfrak{k}=\mathfrak{h o l} \subset \mathfrak{s p}^{\pi}(1) \oplus \mathfrak{s p}^{\pi}(n)$. If $\operatorname{scal}(M) \neq 0$ then the curvature decomposition shows that $\mathfrak{k}^{\mathfrak{k}}$ contains the $\mathfrak{s p}^{\pi}(1)$-factor (see AC1, Lemma 1]), so $\mathfrak{k}=\mathfrak{s p}^{\pi}(1) \oplus \mathfrak{k}^{\prime}$. The isomorphism $T_{o} M \cong \mathfrak{m}$ identifies the quaternionic structure $Q_{o} \subset \operatorname{End}\left(T_{o} M\right)$ with $\left.\operatorname{ad}_{\mathfrak{s p}^{\pi}(1)}\right|_{\mathfrak{m}}$. Now choose a para complex structure $J_{o} \in Q_{o}$. This corresponds to an element $\xi \in \mathfrak{s p}^{\pi}(1) \subset \mathfrak{k}$. By [AC1, Lemma 3 and 4], $\mathfrak{g}$ is simple and $\xi$ corresponds to a long root $\mu$ with respect to a Cartan subalgebra $\mathfrak{a} \subset \mathfrak{g}^{\mathbb{C}}$ in the sense that $\forall \alpha \in \mathfrak{a}^{*}: \alpha(\xi)=2 \frac{\langle\alpha, \mu\rangle}{\langle\mu, \mu\rangle}$. In particular, $\alpha(\xi) \in\{0, \pm 1, \pm 2\}$, so $\xi$ defines a depth two grading of $\mathfrak{g}$, and $\mathfrak{g}_{ \pm 2}$ are the one-dimensional root spaces $\mathfrak{g}_{ \pm \mu}$. To see that [,]: $\mathfrak{g}_{ \pm 1} \times \mathfrak{g}_{ \pm 1} \rightarrow \mathfrak{g}_{ \pm 2}$ 
is nondegenerate, we remark that for each root $\alpha \in \mathfrak{a}^{*}$ with $\alpha(\xi)= \pm 1$, we have $\left[\mathfrak{g}_{\alpha}, \mathfrak{g}_{-w_{\mu}(\alpha)}\right]=\mathfrak{g}_{ \pm \mu} \neq 0$, where $w_{\mu} \in \mathcal{W}$ denotes the element of the Weyl group that is the reflection through the hyperplane $\{\mu=0\}$. Of course, the nondegeneracy implies that $\mathfrak{g}_{ \pm 1}$ generates $\bigoplus_{\lambda \gtrless 0} \mathfrak{g}_{\lambda}$, so $\xi$ is semi-canonical.

(2): Let $\mathfrak{g}=\mathfrak{g}_{-2} \oplus \mathfrak{g}_{-1} \oplus \mathfrak{g}_{0} \oplus \mathfrak{g}_{1} \oplus \mathfrak{g}_{2}$. Then $\mathfrak{g}_{-2} \oplus \mathbb{R} \xi \oplus \mathfrak{g}_{2}$ is a subalgebra isomorphic to $\mathfrak{s p}^{\pi}(1)$, and we have $\mathfrak{g}_{0}=\mathbb{R} \xi \oplus Z_{\mathfrak{g}}\left(\mathfrak{s p}^{\pi}(1)\right)$. It follows that $\mathfrak{k}=\mathfrak{s p}^{\pi}(1) \oplus \mathfrak{k}^{\prime}$ with $\mathfrak{k}^{\prime}=Z_{\mathfrak{g}}\left(\mathfrak{s} \mathfrak{p}^{\pi}(1)\right)$. This implies that $Q_{0}:=\left.\operatorname{ad}_{\mathfrak{s p}}{ }^{\pi}(1)\right|_{\mathfrak{m}}$ is a para-quaternionic structure on $\mathfrak{m} \cong T_{o} M$, which is invariant under the adjoint action of $\mathfrak{k}$ and therefore under $K_{0}=G_{0}^{\sigma}$. By definition, it is also invariant under $H$, hence under $K \subset H \cdot G_{0}^{\sigma}$. Therefore $Q_{0}$ extends to a $G$-invariant para-quaternionic structure on $T M$.

(3): The twistor space $Z^{Q}$ of para-complex structures is the $G$-fibre bundle $G \times{ }_{K} Z_{o}^{Q}$, where $Z_{o}^{Q}=\left\{q \in Q_{o}: q^{2}=1\right\}$ is the $\operatorname{Sp}^{\pi}(1)$-orbit of $\xi$ in $Q_{o}$. Since $K$ contains the $\operatorname{Sp}^{\pi}(1)$ factor, it acts transitively on $Z_{o}^{Q}$. It follows that $G$ acts transitively on $Z^{Q}$, so $Z^{Q}$ is the homogenous space $G / H^{\prime}$, where $H^{\prime}=K \cap H$ is the centralizer of $\xi$ in $K$. But since $H \subset K$ by assumption, we have $H^{\prime}=H$ and therefore $Z^{Q}=\operatorname{Ad}_{G}(\xi)$.

Birational equivalence of twistor spaces. Let $G$ be a linear algebraic group. We have seen that the twistor space $Z$ is an open subset of $\bar{Z}=G^{\mathbf{c}} / P_{-}^{\mathbf{c}}$, which is a real variety, and that $\exp \left(\mathfrak{n}_{+}^{\mathbf{c}}\right)=$ $N_{+}^{\mathbf{c}} \rightarrow G^{\mathbf{c}} / P_{-}^{\mathbf{c}}$ is an embedding onto a Zariski-open and -dense subset, which induces a birational equivalence $N_{+}^{\mathbf{c}} \sim G^{\mathbf{c}} / P_{-}^{\mathbf{c}}$.

If $Z$ is the twistor space of a para-quaternionic Kähler symmetric space, then $N_{+}^{\mathbf{c}}$ is isomorphic to the (generalized) para-complex Heisenberg group:

Definition 3.5.2. The (real, complex, para-complex) Heisenberg group $\mathrm{H}(n, \mathrm{~K})(\mathrm{K}=\mathbb{R}, \mathbb{C}, \mathbf{C})$ is the group of $(n+2) \times(n+2)$-matrices of the form

$$
\left(\begin{array}{ccccc}
1 & & & & \\
z^{1} & 1 & & & \\
\vdots & & \ddots & & \\
z^{n} & & & 1 & \\
w_{0} & w_{1} & \cdots & w_{n} & 1
\end{array}\right) \in \mathrm{GL}(n+2, \mathrm{~K})
$$

Its Lie algebra is the Heisenberg algebra $\mathfrak{h}(n, \mathrm{~K})$ and consists of the matrices

$$
\left(\begin{array}{ccccc}
0 & & & & \\
u^{1} & 0 & & & \\
\vdots & & \ddots & & \\
u^{n} & & & 0 & \\
v_{0} & v_{1} & \cdots & v_{n} & 0
\end{array}\right) \in \mathfrak{g l}(n+2, \mathrm{~K}) .
$$

REMARK. Note that $\mathrm{H}(n, \mathbf{C}) \cong \mathrm{H}(n, \mathbb{R}) \times \mathrm{H}(n, \mathbb{R})$. 
LEMMA 3.5.3. A Lie algebra is isomorphic to the Heisenberg algebra if and only if it is two-step nilpotent with one-dimensional kernel.

(In the case $\mathrm{K}=\mathbf{C}$ this condition includes that the kernel is a paracomplex subspace.)

Proof. Let $\left(u_{1}, \ldots, u_{n}, v^{0}, \ldots, v^{n}\right)$ be the obvious basis of $\mathfrak{h}(n, \mathrm{~K})$ (i. e. the one which is dual to the above coefficients). Then $\left[v^{a}, u_{b}\right]=$ $\delta_{b}^{a} v^{0}$ for $1 \leq a, b \leq n$, and all other brackets are zero, so obviously $\mathfrak{h}(n, \mathrm{~K})$ is two-step nilpotent with kernel $\mathrm{K} \cdot v^{0}$.

Conversely, let $\mathfrak{g}$ be a two-step nilpotent Lie algebra and let $v^{0}$ be a nonzero (resp. regular, if $\mathrm{K}=\mathbf{C}$ ) element of its one-dimensional kernel. Let $\mathfrak{g}^{\prime}$ a complementary subspace, i. e. $\mathfrak{g}=\mathrm{K} \cdot v^{0} \oplus \mathfrak{g}^{\prime}$. By assumption we have $\left[\mathfrak{g}^{\prime}, \mathfrak{g}^{\prime}\right]=\mathrm{K} \cdot v^{0}$, so the Lie bracket defines a symplectic form $\omega$ on $\mathfrak{g}^{\prime}$ via $[\cdot, \cdot]=\omega(\cdot, \cdot) v^{0}$. Let $\left(v^{a}, u_{b}\right)_{a, b=1 \ldots n}$ be a symplectic basis of $\left(\mathfrak{g}^{\prime}, \omega\right)$ (see Proposition 2.1.2 , then $\left[v^{a}, u_{b}\right]=\delta_{b}^{a} \cdot v^{0}$, which defines an isomorphism $\mathfrak{g} \cong \mathfrak{h}(n, \mathrm{~K})$.

Proposition 3.5.4. $\mathrm{H}(n, \mathrm{~K})$ has a left-invariant contact form given by

$$
d w_{0}-\sum_{a=1}^{n} w_{a} d z^{a} .
$$

Let $\bar{Z}=G^{\mathbf{c}} / P_{-}^{\mathbf{c}}$ be the closed twistor space associated to a para-quaternionic Kähler structure. Let $N_{+}^{\mathbf{c}}$ be the unipotent radical of $P_{+}^{\mathbf{c}}$. Then $N_{+}^{\mathbf{c}} \cong \mathrm{H}(n, \mathbf{C})$ (as real linear algebraic groups). This isomorphism is biholomorphic and identifies the contact distribution on $\mathrm{H}(n, \mathbf{C})$ with the horizontal distribution on the orbit of $N_{+}^{\mathbf{c}}$ in $\bar{Z}$. Moreover, it extends to a birational equivalence $N_{+}^{\mathrm{c}} \sim \mathrm{H}(n, \mathbf{C})$.

Proof. By Proposition 3.5.1 we have $\mathfrak{n}_{+}^{\mathbf{c}}=\mathfrak{g}_{1}^{\mathbf{c}} \oplus \mathfrak{g}_{2}^{\mathbf{c}}$, and the Lie bracket [,] $\mathfrak{g}_{1}^{\mathbf{c}} \times \mathfrak{g}_{1}^{\mathbf{c}} \rightarrow \mathfrak{g}_{2}^{\mathbf{c}}$ is nondegenerate. So $\mathfrak{n}_{+}^{\mathbf{c}}$ is two-step nilpotent with one-dimensional kernel $\mathfrak{g}_{2}^{\mathbf{c}}$. This shows that $\mathfrak{n}_{+}^{\mathbf{c}} \cong \mathfrak{h}(n, \mathbf{C})$, or equivalently $\mathfrak{n}_{+} \times \mathfrak{n}_{-} \cong \mathfrak{h}(n, \mathbb{R}) \times \mathfrak{h}(n, \mathbb{R})$. Exponentiating this, we get an isomorphism of the groups $N_{+} \times N_{-} \cong H(n, \mathbb{R}) \times H(n, \mathbb{R})$. This isomorphism is algebraic (i.e. has polynomial components) because both the exponential and logarithm series are finite. Obviously, it is a biholomorphism. To show that the contact distribution on $N_{+}^{\mathrm{c}}$ obtained through this isomorphism is mapped to the horizontal distribution by the map $\pi: N_{+}^{\mathbf{c}} \rightarrow G^{\mathbf{c}} / P_{-}^{\mathbf{c}}$, it suffices to remark that both distributions are $N_{+}^{\mathbf{c}}$-invariant and coincide at the point $e \in N_{+}^{\mathbf{c}}$, where they are given by the linear subspace $\mathfrak{g}_{1}^{\mathbf{c}} \subset \mathfrak{n}_{+}^{\mathbf{c}}$. The statement about the birational equivalence has already been made in Theorem 3.3 .12 . 
Thus we have shown a refinement of Theorem 2.1.11, The Darboux coordinates can be constructed explicitly by writing down the isomorphism $N_{+}^{\mathbf{c}} \cong H(n, \mathbf{C})$. They are defined on the open dense subset $\pi\left(N_{+}^{\mathbf{c}}\right) \cap Z$ of $Z$.

THEOREM 3.5.5. Let $Z$ be the twistor space of a para-quaternionic Kähler symmetric space. Then there is an open dense subset $U \subset Z$ and para-holomorphic coordinates $\left(z^{1}, \ldots, z^{n}, w_{0}, \ldots, w_{n}\right): U \rightarrow \mathbf{C}^{2 n+1}$ such that the horizontal distribution is given by $\operatorname{ker}\left(d w_{0}-\sum_{a=1}^{n} w_{a} d z^{a}\right)$.

Now let $Z^{\prime}$ be the twistor space of another para-quaternionic Kähler symmetric space of the same dimension, then we can put the birational maps together:

$$
G^{\prime \mathbf{c}} / P_{-}^{\prime \mathbf{c}} \leftarrow N_{+}^{\prime \mathbf{c}} \cong H(n, \mathbf{C}) \cong N_{+}^{\mathbf{c}} \rightarrow G^{\mathbf{c}} / P_{-}^{\mathbf{c}}
$$

This proves:

THEOREM 3.5.6. Let $\bar{Z}=G^{\mathbf{c}} / P_{-}^{\mathbf{c}}$ and $\bar{Z}^{\prime}=G^{\mathbf{c}} / P_{-}^{\prime \mathbf{c}}$ be two closed twistor spaces associated to para-quaternionic Kähler symmetric spaces of the same dimension. Then there is a biholomorphic map between Zariski-open and-dense subsets of $\bar{Z}$ and $\bar{Z}^{\prime}$, which preserves the horizontal distribution. This morphism induces a birational equivalence $\bar{Z} \sim \overline{Z^{\prime}}$.

This allows us to construct para-pluriharmonic maps into any paraquaternionic Kähler symmetric space by transferring the construction from $\mathrm{Gr}_{2}\left(\mathbf{C}^{n+2}\right)$ (see section 3.4).

\subsection{The classical para-quaternionic Kähler symmetric spaces}

The simply connected para-quaternionic Kähler symmetric spaces have been classified by Alekseevsky and Cortés [AC1], see also [DJS]:

TheOREM (Alekseevsky, Cortés). Let $M=G / K$ be a simply connected para-quaternionic Kähler symmetric space. Then $M$ is one of the following spaces: 
A)

$$
\frac{\mathrm{SL}(n+2, \mathbb{R})}{\mathrm{S}\left(\mathrm{GL}^{+}(2, \mathbb{R}) \times \mathrm{GL}^{+}(n, \mathbb{R})\right)}, \frac{\mathrm{SU}(p+1, q+1)}{\mathrm{S}(\mathrm{U}(1,1) \times \mathrm{U}(p, q))}
$$

$B D)$

$$
\frac{\mathrm{SO}^{+}(p+2, q+2)}{\mathrm{SO}^{+}(2,2) \times \mathrm{SO}^{+}(p, q)}, \frac{\mathrm{SO}^{*}(2 n+4)}{\mathrm{SO}^{*}(4) \times \mathrm{SO}^{*}(2 n)}
$$

C)

$$
\frac{\mathrm{Sp}(2 n+2, \mathbb{R})}{\mathrm{Sp}(2, \mathbb{R}) \times \operatorname{Sp}(2 n, \mathbb{R})}
$$

$$
\frac{\mathrm{E}_{6(6)}}{\mathrm{SL}(2, \mathbb{R}) \times \mathrm{SL}(6, \mathbb{R})}, \frac{\mathrm{E}_{6(2)}}{\mathrm{SU}(3,3) \times \mathrm{SU}(1,1)}, \frac{\mathrm{E}_{6(-14)}}{\mathrm{SU}(5,1) \times \mathrm{SU}(1,1)}
$$

$$
\begin{aligned}
& \frac{\mathrm{E}_{7(7)}}{\mathrm{SL}(2, \mathbb{R}) \times \operatorname{Spin}_{0}(6,6)}, \frac{\mathrm{E}_{7(-5)}}{\mathrm{SL}(2, \mathbb{R}) \times \mathrm{SO}^{*}(12)}, \frac{\mathrm{E}_{7(-25)}}{\mathrm{SL}(2, \mathbb{R}) \times \operatorname{Spin}_{0}(10,2)} \\
& \frac{\mathrm{E}_{8(8)}}{\mathrm{SL}(2, \mathbb{R}) \times \mathrm{E}_{7(7)}}, \frac{\mathrm{E}_{8(-24)}}{\mathrm{SL}(2, \mathbb{R}) \times \mathrm{E}_{7(-25)}}
\end{aligned}
$$$$
\frac{F_{4(4)}}{\mathrm{SL}(2, \mathbb{R}) \times \operatorname{Sp}(6, \mathbb{R})}
$$$$
\frac{\mathrm{G}_{2(2)}}{\mathrm{SO}(2,2)}
$$

We want to describe the spaces belonging to the classical groups more in detail. However, to apply the results of this chapter, it is useful to consider spaces of the form $G /\left(H \cdot G_{0}^{\sigma}\right)$ rather than their universal covers. We will find that these are the following Grassmannians:

$\left(\mathrm{K}^{p, q}(\mathrm{~K}=\mathbb{R}, \mathbb{C})\right.$ denotes the vector space $\mathrm{K}^{p+q}$, endowed with an indefinite pseudo-Euclidean or -hermitian scalar product of signature $(p, q)$. A subspace of signature $\left(p^{\prime}, q^{\prime}\right)$ is called $\left(p^{\prime}, q^{\prime}\right)$-subspace.)

(1) $\frac{\mathrm{SL}(n+2, \mathbb{R})}{\mathrm{S}(\mathrm{GL}(2, \mathbb{R}) \times \mathrm{GL}(n, \mathbb{R}))} \cong \mathrm{Gr}_{2}\left(\mathbf{C}^{n+2}\right)$, the set of nondegenerate 2dimensional para-complex subspaces of $\mathbf{C}^{n+2}$

(2) $\frac{\mathrm{SU}(p+1, q+1)}{\mathrm{S}(\mathrm{U}(1,1) \times \mathrm{U}(p, q))} \cong \mathrm{Gr}_{1,1}\left(\mathbb{C}^{p+1, q+1}\right)$, the set of complex $(1,1)$-subspaces of $\mathbb{C}^{p+1, q+1}$

(3) $\frac{\mathrm{SO}(p+2, q+2)}{\mathrm{SO}(2,2) \times \mathrm{SO}(p, q)} \cong \mathrm{Gr}_{2,2}^{\text {or }}\left(\mathbb{R}^{p+2, q+2}\right)$, the set of oriented real $(2,2)$-subspaces of $\mathbb{R}^{p+2, q+2}$

(4) $\frac{\mathrm{SO}^{*}(2 n+4)}{\mathrm{SO}^{*}(4) \times \mathrm{SO}^{*}(2 n)} \cong \mathrm{Gr}_{2,2}^{\mathbb{H}}\left(\mathbb{C}^{n+2, n+2}\right)$, the set of those complex $(2,2)$-subspaces of $\mathbb{C}^{n+2, n+2}$ which are invariant under a certain quaternionic structure.

(5) $\frac{\mathrm{Sp}(2 n+2, \mathbb{R})}{\mathrm{Sp}(2, \mathbb{R}) \times \operatorname{Sp}(2 n, \mathbb{R})} \cong \mathbf{H P}^{n}$, the para-quaternionic projective space.

Following the procedure described in the preceding section, we will construct Darboux coordinates on their twistor spaces, which in turn will give us a description of the isotropic para-pluriharmonic maps. (In the cases (1) and (5) it is natural to use $\mathbf{C}$-valued coordinates, whereas we will use $(\mathbb{R} \times \mathbb{R})$-valued coordinates in the cases (2)-(4).)

$$
\text { 3.6.1. } \mathrm{SL}(n+2, \mathbb{R}) / \mathrm{S}(\mathrm{GL}(2, \mathbb{R}) \times \mathrm{GL}(n, \mathbb{R})) \text {. }
$$

Let $G=\mathrm{SU}^{\pi}(n+2) \cong \mathrm{SL}(n+2, \mathbb{R})$. The Lie algebra of $G$ is

$$
\mathfrak{g}=\left\{X \in \mathfrak{g l}(n+2, \mathbf{C}): X^{*}+X=0, \operatorname{tr}(X)=0\right\}
$$


In section 3.4 we have seen that the canonical element

$$
\xi:=\left(\begin{array}{ccc}
-j & & \\
& \mathbf{0}_{n} & \\
& & j
\end{array}\right)
$$

leads to the twistor fibration $G / H \rightarrow G / K$ (with $K=G^{\sigma}=H \cdot G_{0}^{\sigma}$ )

$$
\frac{\mathrm{SU}^{\pi}(n+2)}{\mathrm{S}\left(\mathrm{U}^{\pi}(1) \times \mathrm{U}^{\pi}(n) \times \mathrm{U}^{\pi}(1)\right)} \rightarrow \frac{\mathrm{SU}^{\pi}(n+2)}{\mathrm{S}\left(\mathrm{U}^{\pi}(2) \times \mathrm{U}^{\pi}(n)\right)} .
$$

From section 3.4 we also know that the fibration $G / H \rightarrow G / K$ can be identified with

$$
\mathrm{F}_{1, n, 1}\left(\mathbf{C}^{n+2}\right) \rightarrow \operatorname{Gr}_{2}\left(\mathbf{C}^{n+2}\right)
$$

where $\operatorname{Gr}_{2}\left(\mathbf{C}^{n+2}\right)$ is a para-complex Grassmannian and $Z=\mathrm{F}_{1, n, 1}\left(\mathbf{C}^{n+2}\right)$ the space of nondegenerate $(1, n, 1)$-flags in $\mathbf{C}^{n+2}$.

We have also proven that $Z$ is an open subset of $\bar{Z}=G^{\mathbf{c}} / P_{-}^{\mathbf{c}}$. In the present case, $G^{\mathbf{c}}=\mathrm{SL}(n+2, \mathbf{C})$, and $P_{-}^{\mathbf{c}}$ is the group of upper block diagonal matrices of the form

$$
\left(\begin{array}{l|l|l}
\bullet & \bullet & \bullet \\
\hline 0 & \bullet & \bullet \\
\hline 0 & 0 & \bullet
\end{array}\right) \in \mathrm{SL}(1+n+1, \mathbf{C}) .
$$

Thus, $\bar{Z}$ is the space of all $(1, n, 1)$-flags in $\mathbf{C}^{n+2}$ : Such a flag is a sequence $0 \subsetneq W_{1} \subsetneq W_{2} \subsetneq \mathbf{C}^{n+2}$, where $W_{1}$ and $W_{2}$ are para-complex subspaces (possibly with degenerate scalar product) of dimension 1 and $1+n$, respectively. The map $G^{\mathbf{c}} / P_{-}^{\mathbf{c}} \stackrel{\sim}{\rightarrow} \bar{Z}$ is defined as follows: Given an element of $G^{\mathbf{c}}$, let $W_{1}$ be the $\mathbf{C}$-span of its first column and $W_{2}$ the $\mathbf{C}$-span of the first $1+n$ columns. This map is easily seen to be surjective and invariant under multiplication by elements of $P_{-}^{\mathbf{c}}$ from the right.

The unipotent radical $N_{+}^{\mathbf{c}}$ of $P_{+}^{\mathbf{c}}$ is the para-complex Heisenberg group $\mathrm{H}(n, \mathbf{C})$. We want to determine the orbit of $N_{+}^{\mathbf{c}}$ in $G^{\mathbf{c}} / P_{-}^{\mathbf{c}}:$ By definition it consists of those $g \cdot P_{-}^{\mathbf{c}}$, such that $g \in N_{+}^{\mathbf{c}} \cdot P_{-}^{\mathbf{c}}$, i. e.

$$
\left(\begin{array}{ccc}
g_{1,1} & \cdots & g_{1, n+2} \\
\vdots & \ddots & \vdots \\
g_{n+2,1} & \cdots & g_{n+2, n+2}
\end{array}\right)=\left(\begin{array}{c|c|c}
1 & 0 & 0 \\
\hline \bullet & \mathbf{1}_{n} & 0 \\
\hline \bullet & \bullet & 1
\end{array}\right) \cdot\left(\begin{array}{c|c|c}
\bullet & \bullet & \bullet \\
\hline 0 & \bullet & \bullet \\
\hline 0 & 0 & \bullet
\end{array}\right) .
$$

A necessary condition is that the square blocks on the diagonal of the right factor are invertible. From this it follows after short calculation that

$$
\operatorname{det}\left(\begin{array}{ccc}
g_{1,1} & \cdots & g_{1, n+1} \\
\vdots & \ddots & \vdots \\
g_{n+1,1} & \cdots & g_{n+1, n+1}
\end{array}\right) \in \mathbf{C}^{*}
$$


The Gaussian elimination algorithm shows that this condition is also sufficient:

Since $g_{1,1}$ is invertible, we can annihilate the rest of the first column of $g$ by adding multiples of the first row to the other rows. This is achieved by left multiplication of a suitable matrix, so we obtain

$$
\left(\begin{array}{c|c|c}
1 & 0 & 0 \\
\hline \bullet & \mathbf{1}_{n} & 0 \\
\hline \bullet & 0 & 1
\end{array}\right) \cdot g=\left(\begin{array}{c|c|c}
\bullet & \bullet & \bullet \\
\hline 0 & \bullet & \bullet \\
\hline 0 & \bullet & \bullet
\end{array}\right)
$$

This does not change the determinant of the upper left $(n+$ $1) \times(n+1)$ submatrix, which is invertible by equation $(12)$. It follows that the middle $n \times n$-block on the right hand side is invertible, so we can eliminate the middle block in the last row:

$$
\left(\begin{array}{c|c|c}
1 & 0 & 0 \\
\hline 0 & \mathbf{1}_{n} & 0 \\
\hline 0 & \bullet & 1
\end{array}\right) \cdot\left(\begin{array}{c|c|c}
\bullet & \bullet & \bullet \\
\hline 0 & \bullet & \bullet \\
\hline 0 & \bullet & \bullet
\end{array}\right)=\left(\begin{array}{c|c|c}
\bullet & \bullet & \bullet \\
\hline 0 & \bullet & \bullet \\
\hline 0 & 0 & \bullet
\end{array}\right) .
$$

This yields the desired decomposition of $g$.

From Corollary 1.1.7 it follows that the equations (11f) define an $\mathbb{R}$ Zariki-open subset of $\bar{Z}$, as claimed in Theorem 3.3.12.

It remains to give a description of the isotropic para-pluriharmonic maps:

The coordinates on $N_{+}^{\mathrm{c}}$,

$$
\left(\begin{array}{c|c|c}
1 & & \\
\hline \boldsymbol{z} & \mathbf{1}_{n} & \\
\hline w_{0} & \boldsymbol{w}^{t} & 1
\end{array}\right)
$$

$\boldsymbol{z}=\left(z^{1}, \ldots, z^{n}\right)^{t}, \boldsymbol{w}=\left(w_{1}, \ldots, w_{n}\right)^{t}$, yield holomorphic Darboux coordinates on $\pi\left(N_{+}^{\mathbf{c}}\right) \subset G^{\mathbf{c}} / P_{-}^{\mathbf{c}}$. With respect to these coordinates, a holomorphic map

$$
\begin{aligned}
F: \mathbf{C}^{n} \supset U_{z} & \rightarrow G^{\mathbf{c}} / P_{-}^{\mathbf{c}} \\
\left(z^{1}, \ldots, z^{n}\right) & \mapsto\left(z^{1}, \ldots, z^{n}, w_{0}, \ldots, w_{n}\right)
\end{aligned}
$$

is horizontal, if there is a holomorphic function $h: U_{z} \rightarrow \mathbf{C}$, such that

$$
\begin{aligned}
w_{0} & =h\left(z^{1}, \ldots, z^{n}\right) \\
w_{a} & =\partial h / \partial z^{a}, a=1 \ldots n
\end{aligned}
$$

This holomorphic horizontal map projects onto the isotropic parapluriharmonic map

$$
\begin{aligned}
f=\pi \circ F: U_{z} & \rightarrow \operatorname{Gr}_{2}\left(\mathbf{C}^{n+2}\right) \\
\left(z^{1}, \ldots, z^{n}\right) & \mapsto W_{1} \oplus W_{2}^{\perp},
\end{aligned}
$$


where

$W_{1}=\mathbf{C} \cdot\left(\begin{array}{c}1 \\ z^{1} \\ \vdots \\ z^{n} \\ h\end{array}\right), W_{2}=\operatorname{span}_{\mathbf{C}}\left\{\left(\begin{array}{c}1 \\ z^{1} \\ \vdots \\ z^{n} \\ h\end{array}\right),\left(\begin{array}{c}0 \\ 1 \\ \vdots \\ 0 \\ \frac{\partial h}{\partial z^{1}}\end{array}\right), \ldots,\left(\begin{array}{c}0 \\ 0 \\ \vdots \\ 1 \\ \frac{\partial h}{\partial z^{n}}\end{array}\right)\right\}$.

This is what we have seen in section 3.4 (neglecting that we can change this map by a biholomorphism $\mathbf{C}^{n} \supset U_{z} \stackrel{\sim}{\rightarrow} \tilde{U}_{z} \subset \mathbf{C}^{n}$ ).

\subsection{2. $\mathrm{SU}(p+1, q+1) / \mathrm{S}(\mathrm{U}(1,1) \times \mathrm{U}(p, q))$.}

Let $G=\mathrm{SU}(p+1, q+1)$ be the group of endomorphisms of $\mathbb{C}^{n+2}$, $n=p+q$, with unit determinant which preserve the hermitian scalar product given by the matrix

$$
E:=\left(\begin{array}{l|ll|l} 
& & & 1 \\
\hline & \mathbf{1}_{p} & & \\
& & -\mathbf{1}_{q} & \\
\hline 1 & & &
\end{array}\right)
$$

Its Lie algebra is $\mathfrak{g}=\mathfrak{s u}(p+1, q+1)=\left\{X \in \mathfrak{s l}(n+2, \mathbb{C}): X^{*} E+E X=0\right\}$ and consists of traceless matrices of the form

$$
\left(\begin{array}{c|cc|c}
a & -C^{*} & D^{*} & c \\
\hline A & \mathfrak{u}(p, q) & C \\
B & D \\
\hline b & -A^{*} \quad B^{*} & -\bar{a}
\end{array}\right) \in \mathfrak{s l}(1+n+1, \mathbb{C}), b, c \in i \mathbb{R} .
$$

The canonical element

$$
\xi:=\left(\begin{array}{l|l|l}
-1 & & \\
\hline & \mathbf{0}_{p+q} & \\
\hline & & 1
\end{array}\right)
$$

leads to the twistor fibration

$$
\frac{\mathrm{SU}(p+1, q+1)}{\mathrm{S}\left(\mathrm{U}(p, q) \times \mathbb{C}^{*}\right)} \rightarrow \frac{\mathrm{SU}(p+1, q+1)}{\mathrm{S}(\mathrm{U}(p, q) \times \mathrm{U}(1,1))}
$$

(where $\mathbb{C}^{*}=\left\{\left(\begin{array}{cc}a & 0 \\ 0 & 1 / \bar{a}\end{array}\right): a \in \mathbb{C}^{*}\right\} \subset \mathrm{U}(1,1)$ ).

The coset space $\mathrm{SU}(p+1, q+1) / \mathrm{S}(\mathrm{U}(p, q) \times \mathrm{U}(1,1))$ can be identified with the Grassmannian $\mathrm{Gr}_{1,1}\left(\mathbb{C}^{p+1, q+1}\right)$ of 2-dimensional complex subspaces $V \subset \mathbb{C}^{n+2}$ which have signature $(1,1)$ with respect to the above pseudo-hermitian metric; an element of $G$ is sent to the subspace $V$ that is spanned by the first and the last column. The twistor fibre over $V \subset \mathbb{C}^{n+2}$ is the set of compatible para-complex structures on $V$, i. e. the set of decompositions $V=V^{+} \oplus V^{-}$into complementary complex (totally) degenerate spaces. 
The subgroups $P_{ \pm}$and $N_{ \pm}$are the groups of lower or upper block diagonal matrices, where the groups $N_{ \pm}$have unit matrices on the diagonal. The homogenous space $\left(G / P_{-}\right) \times\left(G / P_{+}\right)$is the space $\bar{Z}$ of all pairs $\left(V^{+}, V^{-}\right)$of one-dimensional degenerate spaces $V^{ \pm} \subset \mathbb{C}^{p+1, q+1}$. The map $G \times G \rightarrow \bar{Z}$ sends a pair $\left(g_{+}, g_{-}\right)$to $\left(V^{+}, V^{-}\right)$, where $V^{+}$is the span of the first column of $g_{+}$and $V^{-}$the span of the last column of $g_{-}$.

As before, the algebra $\mathfrak{n}_{+}$consists of the strictly lower block diagonal matrices. A Lie algebra isomorphism $\mathfrak{h}(p+q, \mathbb{R}) \stackrel{\sim}{\rightarrow} \mathfrak{n}_{+}$is given by

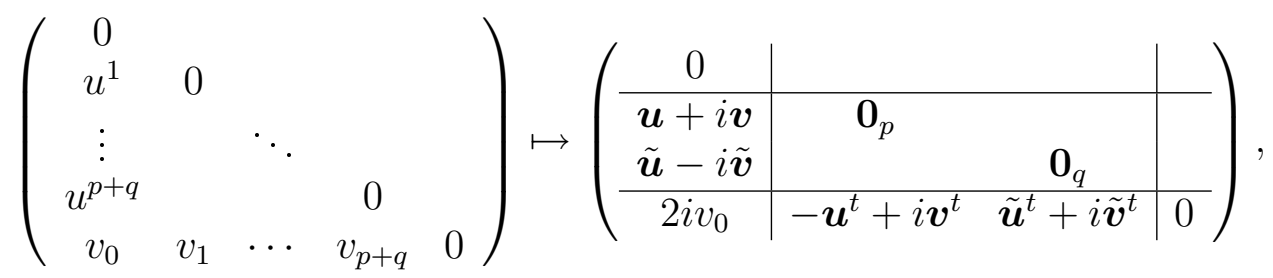

where $\boldsymbol{u}=\left(u^{1}, \ldots, u^{p}\right)^{t}, \tilde{\boldsymbol{u}}=\left(u^{p+1}, \ldots u^{p+q}\right)^{t}, \boldsymbol{v}=\left(v_{1}, \ldots, v_{p}\right)^{t}, \tilde{\boldsymbol{v}}=$ $\left(v_{p+1}, \ldots v_{p+q}\right)^{t}$.

Exponentiating this, we obtain an isomorphism $\mathrm{H}(p+q, \mathbb{R}) \stackrel{\sim}{\rightarrow} N_{+}$, and Darboux coordinates on $N_{+}$:

$$
\left(\begin{array}{c|cc|c}
1 & & & \\
\hline \boldsymbol{z}_{+}+i \boldsymbol{w}_{+} & \mathbf{1}_{p} & 0 & \\
\tilde{\boldsymbol{z}}_{+}-i \tilde{\boldsymbol{w}}_{+} & 0 & \mathbf{1}_{q} & \\
\hline \frac{1}{2}\left(\boldsymbol{z}_{+}^{t} \boldsymbol{z}_{+}-\boldsymbol{w}_{+}^{t} \boldsymbol{w}_{+}+\tilde{\boldsymbol{z}}_{+}^{t} \tilde{\boldsymbol{z}}_{+}+\tilde{\boldsymbol{w}}_{+}^{t} \tilde{\boldsymbol{w}}_{+}\right)+i\left(2 w_{0}^{+}-\boldsymbol{z}_{+}^{t} \boldsymbol{w}_{+}-\tilde{\boldsymbol{z}}_{+}^{t} \tilde{\boldsymbol{w}}_{+}\right) & -\boldsymbol{z}_{+}^{t}+i \boldsymbol{w}_{+}^{t} & \tilde{\boldsymbol{z}}_{+}^{t}-i \tilde{\boldsymbol{w}}_{+}^{t} & 1
\end{array}\right),
$$

where $\boldsymbol{z}_{+}=\left(z_{+}^{1}, \ldots, z_{+}^{p}\right)^{t}, \tilde{\boldsymbol{z}}_{+}=\left(z_{+}^{p+1}, \ldots, z_{+}^{p+q}\right)^{t}, \boldsymbol{w}_{+}=\left(w_{1}^{+}, \ldots, w_{p}^{+}\right)^{t}$, $\tilde{\boldsymbol{w}}_{+}=\left(w_{p+1}^{+}, \ldots, w_{p+q}^{+}\right)^{t}$.

In the same way for $N_{-}$:

$$
\left(\begin{array}{c|cc|c}
1 & -\boldsymbol{z}_{-}^{t}+i \boldsymbol{w}_{-}^{t} & \tilde{\boldsymbol{z}}_{-}^{t}-i \tilde{\boldsymbol{w}}_{-}^{t} & \frac{1}{2}\left(-\boldsymbol{z}_{-}^{t} \boldsymbol{z}_{-}-\boldsymbol{w}_{-}^{t} \boldsymbol{w}_{-}+\tilde{\boldsymbol{z}}_{-}^{t} \tilde{\boldsymbol{z}}_{-}+\tilde{\boldsymbol{w}}_{-}^{t} \tilde{\boldsymbol{w}}_{-}\right)+i\left(2 w_{0}^{-}-\boldsymbol{z}_{-}^{t} \boldsymbol{w}_{-}-\tilde{\boldsymbol{z}}_{-}^{t} \tilde{\boldsymbol{w}}_{-}\right) \\
\hline & \mathbf{1}_{p} & 0 & \boldsymbol{z}_{-}+i \boldsymbol{w}_{-} \\
& 0 & \mathbf{1}_{q} & \tilde{\boldsymbol{z}}_{-}-i \tilde{\boldsymbol{w}}_{-} \\
\hline & & & 1
\end{array}\right)
$$

Therefore, the isotropic para-pluriharmonic maps are of the form

$$
\begin{aligned}
\mathbf{C}^{n} \cong \mathbb{R}^{n} \times \mathbb{R}^{n} \supset U_{z} & \rightarrow \operatorname{Gr}_{(1,1)}\left(\mathbb{C}^{p+1, q+1}\right) \\
\left(z_{ \pm}^{1}, \ldots, z_{ \pm}^{n}\right) & \rightarrow V^{+} \oplus V^{-}
\end{aligned}
$$

where $V_{+}$is the span of the first column of $N_{+}$and $V_{-}$the span of the last column of $N_{-}$(note that these vectors are degenerate):

$$
V_{+}=\mathbb{C} \cdot\left(\begin{array}{c}
1 \\
\boldsymbol{z}_{+}+i \boldsymbol{w}_{+} \\
\tilde{\boldsymbol{z}}_{+}-i \tilde{\boldsymbol{w}}_{+} \\
\frac{1}{2}\left(-\boldsymbol{z}_{+}^{t} \boldsymbol{z}_{+}-\boldsymbol{w}_{+}^{t} \boldsymbol{w}_{+}+\tilde{\boldsymbol{z}}_{+}^{t} \tilde{\boldsymbol{z}}_{+}+\tilde{\boldsymbol{w}}_{+}^{t} \tilde{\boldsymbol{w}}_{+}\right)+i\left(2 w_{0}^{+}-\boldsymbol{z}_{+}^{t} \boldsymbol{w}_{+}-\tilde{\boldsymbol{z}}_{+}^{t} \tilde{\boldsymbol{w}}_{+}\right)
\end{array}\right)
$$


$V_{-}=\mathbb{C} \cdot\left(\begin{array}{c}\frac{1}{2}\left(-\boldsymbol{z}_{-}^{t} \boldsymbol{z}_{-}-\boldsymbol{w}_{-}^{t} \boldsymbol{w}_{-}+\tilde{\boldsymbol{z}}_{-}^{t} \tilde{\boldsymbol{z}}_{-}+\tilde{\boldsymbol{w}}_{-}^{t} \tilde{\boldsymbol{w}}_{-}\right)+i\left(2 w_{0}^{-}-\boldsymbol{z}_{-}^{t} \boldsymbol{w}_{-}-\tilde{\boldsymbol{z}}_{-}^{t} \tilde{\boldsymbol{w}}_{-}\right) \\ \boldsymbol{z}_{-}+i \boldsymbol{w}_{-} \\ \tilde{\boldsymbol{z}}_{-}-i \tilde{\boldsymbol{w}}_{-} \\ 1\end{array}\right)$

and where $w_{0}^{ \pm}=h_{ \pm}\left(z_{ \pm}^{1}, \ldots, z_{ \pm}^{n}\right), w_{a}^{ \pm}=\partial h_{ \pm} / \partial z_{ \pm}^{a}$ for a para-holomorphic function $h=h_{+} \times h_{-}: \mathbb{R}^{n} \times \mathbb{R}^{n} \supset U \rightarrow \mathbb{R} \times \mathbb{R}$.

\subsection{3. $\mathrm{SO}(p+2, q+2) / \mathrm{SO}(p, q) \times \mathrm{SO}(2,2)$.}

Let $G=\mathrm{SO}(p+2, q+2)$ be the group of endomorphism of $\mathbb{R}^{p+q+4}$ which preserve the pseudo-Riemannian metric given by the matrix

$$
E=\left(\begin{array}{l|ll|l} 
& & & \mathbf{1}_{2} \\
\hline & \mathbf{1}_{p} & & \\
& & -\mathbf{1}_{q} & \\
\hline \mathbf{1}_{2} & & &
\end{array}\right)
$$

The Lie algebra $\mathfrak{g} \cong \mathfrak{s o}(p+2, q+2)$ consists of the $E$-skew matrices; these are of the form

$$
\left(\begin{array}{c|cc|c}
a & -C^{t} & D^{t} & c \\
\hline A & \mathfrak{s} o(p, q) & C \\
B & \multicolumn{1}{|c}{{ }^{t}} & B^{t} & -a^{t}
\end{array}\right) \in \mathfrak{g l}(2+n+2) ; b, c \in \mathfrak{o}(2)
$$

The canonical element $\xi:=\left(\begin{array}{l|l|l}-\mathbf{1}_{2} & & \\ \hline & \mathbf{0}_{p+q} & \\ \hline & & \mathbf{1}_{2}\end{array}\right)$ leads to the twistor fibration $G / H \rightarrow G /\left(H \cdot G_{0}^{\sigma}\right)$

$$
\frac{\mathrm{SO}(p+2, q+2)}{\mathrm{SO}(p, q) \times \mathrm{GL}(2, \mathbb{R})} \rightarrow \frac{\mathrm{SO}(p+2, q+2)}{\mathrm{SO}(p, q) \times \mathrm{SO}(2,2)} .
$$

The space $M=\mathrm{SO}(p+2, q+2) / \mathrm{SO}(p, q) \times \mathrm{SO}(2,2)$ can be identified with the Grassmannian $\mathrm{Gr}_{2,2}^{\text {or }}\left(\mathbb{R}^{p+2, q+2}\right)$ of oriented 4-dimensional subspaces of $\mathbb{R}^{p+2, q+2}$ which have signature $(2,2)$ with respect to the above pseudo-Riemannian metric. The map $G \rightarrow M$ is defined as follows: Given an element of $G$, its first two and last two columns form an oriented basis of this (2,2)-subspace.

Note that $M$ has a two-fold quotient, the non-oriented Grassmannian $\mathrm{Gr}_{2,2}\left(\mathbb{R}^{p+2, q+2}\right)=\mathrm{SO}(p+2, q+2) / \mathrm{S}(\mathrm{O}(p, q) \times \mathrm{O}(2,2))$, which is not para-quaternionic Kähler (the para-quaternionic structure is not $\mathrm{S}(\mathrm{O}(p, q) \times \mathrm{O}(2,2))$-invariant $). M$ also has a two-fold cover, the strongly oriented Grassmannian $\mathrm{Gr}_{2,2}^{\text {stror }}\left(\mathbb{R}^{p+2, q+2}\right)=\mathrm{SO}(p+2, q+2) / \mathrm{SO}(p, q) \times$ $\mathrm{SO}^{+}(2,2)$, which is para-quaternionic Kähler, but has no twistor fibration in the sense of Proposition 3.3.4 (in other words, the twistor space defined in Chapter 2 is no adjoint orbit). 
The twistor space $Z=G / H$ is the set of pairs $\left(V^{+}, V^{-}\right)$of 2-dimensional totally degenerate subspaces $V^{ \pm}$of $\mathbb{R}^{p+2, q+2}$, such that the pseudoEuclidean metric is nondegenerate on $V^{+} \oplus V^{-}$(which is therefore a subspace of signature $(2,2))$. The map $Z \rightarrow M$ maps the pair $\left(V^{+}, V^{-}\right)$ to the sum $V^{+} \oplus V^{-}$; note that a canonical orientation on $V^{+} \oplus V^{-}$is obtained through the isomorphism $V^{+} \stackrel{\sim}{\rightarrow}\left(V^{-}\right)^{*}$.

The closed twistor space $\bar{Z}=\left(G / P_{-}\right) \times\left(G / P_{+}\right)$is the space of all pairs $\left(V^{+}, V^{-}\right)$of 2-dimensional totally degenerate subspaces of $\mathbb{R}^{p+2, q+2}$. The map $G \times G \rightarrow \bar{Z}$ sends the pair $\left(g_{+}, g_{-}\right)$to $\left(V^{+}, V^{-}\right)$, where $V^{+}$ is the span of the first two columns of $g_{+}$and $V^{-}$the span of the last two columns of $g_{-}$. This map is $P_{-} \times P_{+}$-invariant and defines an isomorphism $\left(G / P_{-}\right) \times\left(G / P_{+}\right) \stackrel{\sim}{\rightarrow} \bar{Z}$.

An isomorphism $\mathfrak{h}(n, \mathbb{R}) \rightarrow \mathfrak{n}_{+}$is given by

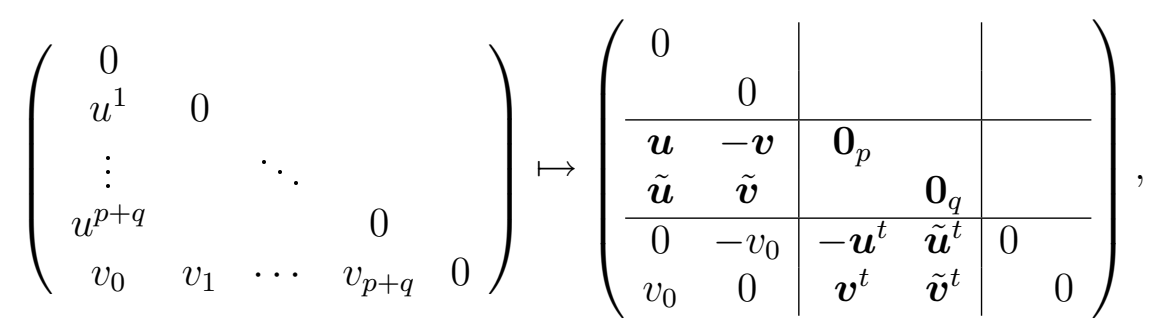

where $\boldsymbol{u}=\left(u^{1}, \ldots, u^{p}\right)^{t}, \tilde{\boldsymbol{u}}=\left(u^{p+1}, \ldots u^{p+q}\right)^{t}, \boldsymbol{v}=\left(v_{1}, \ldots, v_{p}\right)^{t}, \tilde{\boldsymbol{v}}=$ $\left(v_{p+1}, \ldots v_{p+q}\right)^{t}$.

Thus we obtain Darboux coordinates on $N_{+}$:

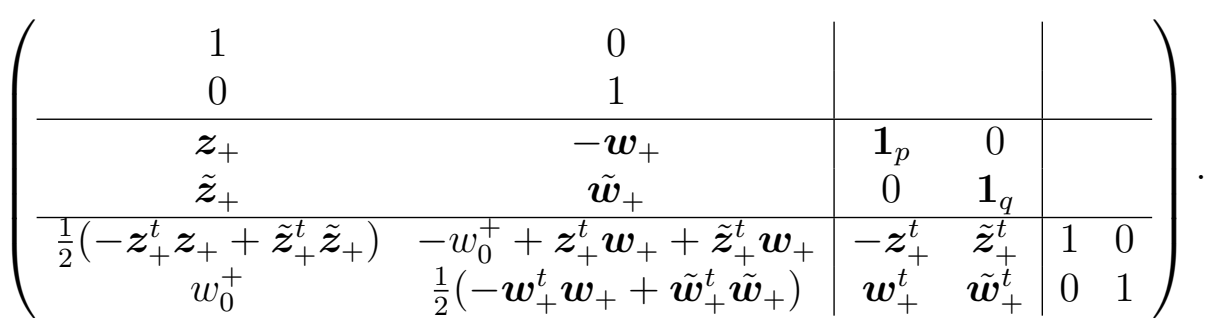

Similarly on $N_{-}$:

$$
\left(\begin{array}{cc|cc|cc}
1 & 0 & -\boldsymbol{z}_{-}^{t} & \tilde{\boldsymbol{z}}_{-}^{t} & \frac{1}{2}\left(-\boldsymbol{z}_{-}^{t} \boldsymbol{z}_{-}+\tilde{\boldsymbol{z}}_{-}^{t} \tilde{\boldsymbol{z}}_{-}\right) & -w_{0}^{-}+\boldsymbol{z}_{-}^{t} \boldsymbol{w}_{-}+\tilde{\boldsymbol{z}}_{-}^{t} \boldsymbol{w}_{-} \\
0 & 1 & \boldsymbol{w}_{-}^{t} & \tilde{\boldsymbol{w}}_{-}^{t} & w_{0}^{-} & \frac{1}{2}\left(-\boldsymbol{w}_{-}^{t} \boldsymbol{w}_{-}+\tilde{\boldsymbol{w}}_{-}^{t} \tilde{\boldsymbol{w}}_{-}\right) \\
\hline & \mathbf{1}_{p} & 0 & \boldsymbol{z}_{-} & -\boldsymbol{w}_{-} \\
& 0 & \mathbf{1}_{q} & \tilde{\boldsymbol{z}}_{-} & \tilde{\boldsymbol{w}}_{-} \\
\hline & & & 1 & 0 \\
& & & 0 & 1
\end{array}\right)
$$

Therefore, the isotropic para-pluriharmonic maps are given by

$$
\begin{aligned}
& \mathbb{R}^{n} \times \mathbb{R}^{n} \supset U \rightarrow \mathrm{Gr}_{2,2}^{\text {or }}\left(\mathbb{R}^{p+2, q+2}\right) \\
& \left(z_{ \pm}^{1}, \ldots, z_{ \pm}^{n}\right) \quad \rightarrow \quad V_{+}\left(z_{+}^{1}, \ldots z_{+}^{n}\right) \oplus V_{-}\left(z_{-}^{1}, \ldots z_{-}^{n}\right)
\end{aligned}
$$


where

$$
\begin{aligned}
& V_{+}=\operatorname{span}_{\mathbb{R}}\left\{\left(\begin{array}{c}
1 \\
0 \\
\boldsymbol{z}_{+} \\
\tilde{\boldsymbol{z}}_{+} \\
\frac{1}{2}\left(-\boldsymbol{z}_{+}^{t} \boldsymbol{z}_{+}+\tilde{\boldsymbol{z}}_{+}^{t} \tilde{\boldsymbol{z}}_{+}\right) \\
w_{0}^{+}
\end{array}\right),\left(\begin{array}{c}
0 \\
1 \\
-\boldsymbol{w}_{+} \\
\tilde{\boldsymbol{w}}_{+} \\
-w_{0}^{+}+\boldsymbol{z}_{+}^{t} \boldsymbol{w}_{+}+\tilde{\boldsymbol{z}}_{+}^{t} \boldsymbol{w}_{+} \\
\frac{1}{2}\left(-\boldsymbol{w}_{+}^{t} \boldsymbol{w}_{+}+\tilde{\boldsymbol{w}}_{+}^{t} \tilde{\boldsymbol{w}}_{+}\right)
\end{array}\right)\right\} \\
& V_{-}=\operatorname{span}_{\mathbb{R}}\left\{\left(\begin{array}{c}
\frac{1}{2}\left(-\boldsymbol{z}_{-}^{t} \boldsymbol{z}_{-}+\tilde{\boldsymbol{z}}_{-}^{t} \tilde{\boldsymbol{z}}_{-}\right) \\
w_{0}^{-} \\
\boldsymbol{z}_{-} \\
\tilde{\boldsymbol{z}}_{-} \\
1 \\
0
\end{array}\right),\left(\begin{array}{c}
-w_{0}^{-}+\boldsymbol{z}_{-}^{t} \boldsymbol{w}_{-}+\tilde{\boldsymbol{z}}_{-}^{t} \boldsymbol{w}_{-} \\
\frac{1}{2}\left(-\boldsymbol{w}_{-}^{t} \boldsymbol{w}_{-}+\tilde{\boldsymbol{w}}_{-}^{t} \tilde{\boldsymbol{w}}_{-}\right) \\
-\boldsymbol{w}_{-} \\
\tilde{\boldsymbol{w}}_{-} \\
0 \\
1
\end{array}\right)\right\}
\end{aligned}
$$

and where $w_{0}^{ \pm}=h_{ \pm}\left(z_{ \pm}^{1}, \ldots, z_{ \pm}^{n}\right), w_{a}^{ \pm}=\partial h_{ \pm} / \partial z_{ \pm}^{a}$ for a para-holomorphic function $h=h_{+} \times h_{-}: \mathbb{R}^{n} \times \mathbb{R}^{n} \supset U \rightarrow \mathbb{R} \times \mathbb{R}$.

\subsection{4. $\mathrm{SO}^{*}(2 n+4) / \mathrm{SO}^{*}(4) \times \mathrm{SO}^{*}(2 n)$.}

Let $\mathbb{H}=\mathbb{R} \oplus i \mathbb{R} \oplus j \mathbb{R} \oplus k \mathbb{R}, i^{2}=j^{2}=-1, k=i j=-j i$, be the algebra of quaternions. We take quaternionic vector spaces to be right $\mathbb{H}$-modules. Accordingly, $\mathbb{H}^{n}$ is the space of column vectors with scalar multiplication on the right, and left multiplication by quaternionic matrices is $\mathbb{H}$-linear. Regarding $\mathbb{H}^{n}$ as the complex vector space $\mathbb{H}^{n}=\mathbb{C}^{n} \oplus j \mathbb{C}^{n} \cong \mathbb{C}^{2 n}$, right multiplication by $j$ becomes a $\mathbb{C}$-antilinear map $r_{j}: \mathbb{C}^{2 n} \rightarrow \mathbb{C}^{2 n}$. A $\mathbb{C}$-linear map $\mathbb{C}^{2 n} \rightarrow \mathbb{C}^{2 n}$ is $\mathbb{H}$-linear, if and only if it commutes with $r_{j}$.

The group $\mathrm{SO}^{*}(2 n)$ - sometimes also denoted $\mathrm{U}^{*}(n, \mathbb{H})$ - is defined as the group of endomorphisms of $\mathbb{H}^{n}$ preserving the skew-hermitian form $\omega(h, k):=h^{*} j k$. Writing $\omega=: \alpha+j \beta$, we obtain maps $\alpha, \beta: \mathbb{C}^{2 n} \times$ $\mathbb{C}^{2 n} \rightarrow \mathbb{C}$, where $\beta$ is $\mathbb{C}$-bilinear symmetric and $\alpha$ is skew-hermitian (whence $i \alpha$ is hermitian). These two are related by $\alpha\left(r_{j}, \cdot\right)=\beta$. It follows that an endomorphism of $\mathbb{C}^{2 n}$ is an element of $\mathrm{SO}^{*}(2 n)$, if and only if it commutes with $r_{j}$ and preserves the hermitian form $i \alpha$. Moreover, we have $l_{j}^{*} \alpha=\overline{r_{j}^{*} \alpha}=-\alpha$. It follows that $i \alpha=: h$ has signature $(n, n)$. Thus we have

$$
\mathrm{SO}^{*}(2 n)=\mathrm{GL}(n, \mathbb{H}) \cap \mathrm{U}(n, n) .
$$


Let $G \cong \mathrm{SO}^{*}(2 n+4)$ be the group of endomorphisms of $\mathbb{C}^{2 n}$ which commute with

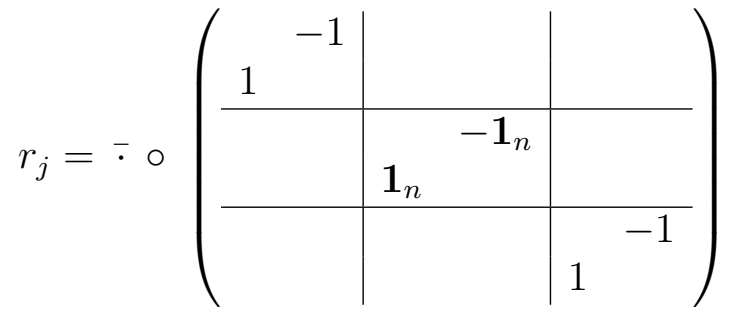

and preserve the indefinite hermitian scalar product $h$ given by the matrix

$$
E:=\left(\begin{array}{ll|ll|ll} 
& & & 1 & \\
& & & -1 \\
\hline & & \mathbf{1}_{n} & & \\
\hline 1 & & & -\mathbf{1}_{n} & \\
\hline & -1 & & &
\end{array}\right)
$$

The Lie algebra $\mathfrak{g} \cong \mathfrak{s o}^{*}(2 n+4)$ consists of matrices of the form

$$
\left(\begin{array}{cc|cc|cc}
a & -\bar{b} & -C^{*} & D^{*} & d & 0 \\
b & \bar{a} & -D^{t} & -C^{t} & 0 & \bar{d} \\
\hline A & -B & \multirow{2}{*}{\mathfrak{s o}^{*}(2 n)} & C & -\bar{D} \\
B & \bar{A} & & D & \bar{C} \\
\hline c & 0 & -A^{*} & B^{*} & -\bar{a} & \bar{b} \\
0 & \bar{c} & -B^{t} & -A^{t} & -b & -a
\end{array}\right) \in \mathfrak{g l}(2 n+4, \mathbb{C}), \quad c, d \in i \mathbb{R} .
$$

The canonical element

$$
\xi:=\left(\begin{array}{l|l|l}
-\mathbf{1}_{2} & & \\
\hline & \mathbf{0}_{2 n} & \\
\hline & & \mathbf{1}_{2}
\end{array}\right)
$$

leads to the twistor fibration

$$
\frac{\mathrm{SO}^{*}(2 n+4)}{\mathrm{GL}(1, \mathbb{H}) \times \mathrm{SO}^{*}(2 n)} \rightarrow \frac{\mathrm{SO}^{*}(2 n+4)}{\mathrm{SO}^{*}(4) \times \mathrm{SO}^{*}(2 n)}
$$

The coset space $\mathrm{SO}^{*}(2 n+4) / \mathrm{SO}^{*}(4) \times \mathrm{SO}^{*}(2 n)$ can be identified with the set of complex subspaces which are $r_{j}$ - invariant and have signature $(2,2)$ with respect to the above pseudo-hermitian product. (In order to see that the $\mathrm{SO}^{*}(2 n+4)$-action on this set is transitive, we have to verify that the orthogonal complement of such a subspace is again $r_{j}$-invariant: This follows from $r_{j}^{*} h=\bar{h}$.) The projection $G \rightarrow G / K$ sends an element of $G$ to the span of its first two and last two columns. 
The isomorphism $\mathfrak{h}(2 n, \mathbb{R}) \stackrel{\sim}{\rightarrow} \mathfrak{n}_{+}$is given by the coordinates

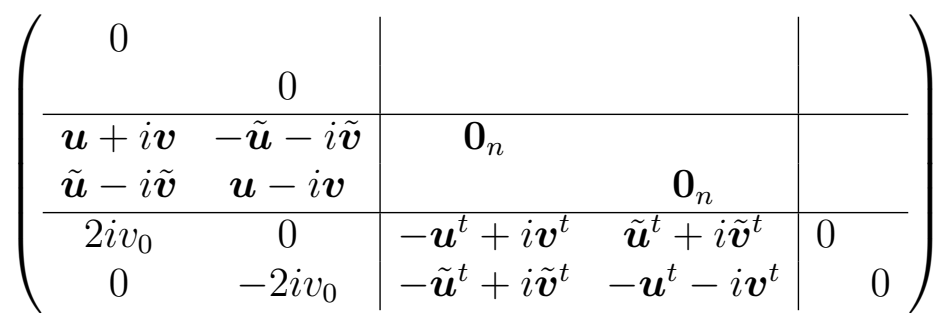

Thus, the isotropic para-pluriharmonic maps

$$
\begin{aligned}
\mathbb{R} \times \mathbb{R} \supset U & \rightarrow \operatorname{Gr}_{2,2}^{\mathbb{H}}\left(\mathbb{C}^{n+2, n+2}\right) \\
\left(z_{ \pm}^{1}, \ldots, z_{ \pm}^{2 n}\right) & \mapsto V_{+}\left(z_{+}^{1}, \ldots, z_{+}^{2 n}\right) \oplus V_{-}\left(z_{-}^{1}, \ldots, z_{-}^{2 n}\right)
\end{aligned}
$$

are given by

$$
\begin{aligned}
& V_{+}=\mathbb{C} \cdot\left(\begin{array}{c}
1 \\
0 \\
\boldsymbol{z}_{+}+i \boldsymbol{w}_{+} \\
\tilde{\boldsymbol{z}}_{+}-i \tilde{\boldsymbol{w}}_{+} \\
\frac{1}{2}\left(-\boldsymbol{z}_{+}^{t} \boldsymbol{z}_{+}-\boldsymbol{w}_{+}^{t} \boldsymbol{w}_{+}+\tilde{\boldsymbol{z}}_{+}^{t} \tilde{\boldsymbol{z}}_{+}+\tilde{\boldsymbol{w}}_{+}^{t} \tilde{\boldsymbol{w}}_{+}\right)+i\left(2 w_{0}^{+}-\boldsymbol{z}_{+}^{t} \boldsymbol{w}_{+}-\tilde{\boldsymbol{z}}_{+}^{t} \tilde{\boldsymbol{w}}_{+}\right) \\
2\left(-\boldsymbol{z}^{t} \tilde{\boldsymbol{z}}-\boldsymbol{w}^{t} \tilde{\boldsymbol{w}}+i\left(\boldsymbol{z}^{t} \tilde{\boldsymbol{w}}-\boldsymbol{w}^{t} \tilde{\boldsymbol{z}}\right)\right)
\end{array}\right) \\
& \oplus \mathbb{C} \cdot\left(\begin{array}{c}
0 \\
1 \\
-\tilde{\boldsymbol{z}}_{+}-i \tilde{\boldsymbol{w}}_{+} \\
\boldsymbol{z}_{+}-i \boldsymbol{w}_{+} \\
2\left(\boldsymbol{z}^{t} \tilde{\boldsymbol{z}}+\boldsymbol{w}^{t} \tilde{\boldsymbol{w}}+i\left(\boldsymbol{z}^{t} \tilde{\boldsymbol{w}}-\boldsymbol{w}^{t} \tilde{\boldsymbol{z}}\right)\right) \\
\frac{1}{2}\left(-\boldsymbol{z}_{+}^{t} \boldsymbol{z}_{+}-\boldsymbol{w}_{+}^{t} \boldsymbol{w}_{+}+\tilde{\boldsymbol{z}}_{+}^{t} \tilde{\boldsymbol{z}}_{+}+\tilde{\boldsymbol{w}}_{+}^{t} \tilde{\boldsymbol{w}}_{+}\right)-i\left(2 w_{0}^{+}-\boldsymbol{z}_{+}^{t} \boldsymbol{w}_{+}-\tilde{\boldsymbol{z}}_{+}^{t} \tilde{\boldsymbol{w}}_{+}\right)
\end{array}\right) \\
& V_{-}=\mathbb{C} \cdot\left(\begin{array}{c}
\frac{1}{2}\left(-\boldsymbol{z}_{-}^{t} \boldsymbol{z}_{-}-\boldsymbol{w}_{-}^{t} \boldsymbol{w}_{-}+\tilde{\boldsymbol{z}}_{-}^{t} \tilde{\boldsymbol{z}}_{-}+\tilde{\boldsymbol{w}}_{-}^{t} \tilde{\boldsymbol{w}}_{-}\right)+i\left(2 w_{0}^{-}-\boldsymbol{z}_{-}^{t} \boldsymbol{w}_{-}-\tilde{\boldsymbol{z}}_{-}^{t} \tilde{\boldsymbol{w}}_{-}\right) \\
2\left(-\boldsymbol{z}_{-}^{t} \tilde{\boldsymbol{z}}_{-}-\boldsymbol{w}_{-}^{t} \tilde{\boldsymbol{w}}_{-}+i\left(\boldsymbol{z}_{-}^{t} \tilde{\boldsymbol{w}}_{-}-\boldsymbol{w}_{-}^{t} \tilde{\boldsymbol{z}}_{-}\right)\right) \\
\boldsymbol{z}_{-}+i \boldsymbol{w}_{-} \\
\tilde{\boldsymbol{z}}_{-}-i \tilde{\boldsymbol{w}}_{-} \\
1 \\
0
\end{array}\right) \\
& \oplus \mathbb{C} \cdot\left(\begin{array}{c}
2\left(\boldsymbol{z}_{-}^{t} \tilde{\boldsymbol{z}}_{-}+\boldsymbol{w}_{-}^{t} \tilde{\boldsymbol{w}}_{-}+i\left(\boldsymbol{z}_{-}^{t} \tilde{\boldsymbol{w}}_{-}-\boldsymbol{w}_{-}^{t} \tilde{\boldsymbol{z}}_{-}\right)\right) \\
\frac{1}{2}\left(-\boldsymbol{z}_{-}^{t} \boldsymbol{z}_{-}-\boldsymbol{w}_{-}^{t} \boldsymbol{w}_{-}+\tilde{\boldsymbol{z}}_{-}^{t} \tilde{\boldsymbol{z}}_{-}+\tilde{\boldsymbol{w}}_{-}^{t} \tilde{\boldsymbol{w}}_{-}\right)-i\left(2 w_{0}^{-}-\boldsymbol{z}_{-}^{t} \boldsymbol{w}_{-}-\tilde{\boldsymbol{z}}_{-}^{t} \tilde{\boldsymbol{w}}_{-}\right) \\
-\tilde{\boldsymbol{z}}_{-}-i \tilde{\boldsymbol{w}}_{-} \\
\boldsymbol{z}_{-}-i \boldsymbol{w}_{-} \\
0 \\
1
\end{array}\right),
\end{aligned}
$$

where $\boldsymbol{z}_{ \pm}=\left(z_{ \pm}^{1}, \ldots, z_{ \pm}^{n}\right), \tilde{\boldsymbol{z}}_{ \pm}=\left(z_{ \pm}^{n+1}, \ldots, z_{ \pm}^{2 n}\right), \boldsymbol{w}_{ \pm}=\left(w_{1}^{ \pm}, \ldots w_{n}^{ \pm}\right)$, $\tilde{\boldsymbol{w}}_{ \pm}=\left(w_{n+1}^{ \pm}, \ldots w_{2 n}^{ \pm}\right)$with $w_{0}^{ \pm}=h_{ \pm}\left(z_{ \pm}^{1}, \ldots, z_{ \pm}^{n}\right), w_{a}^{ \pm}=\partial h_{ \pm} / \partial z_{ \pm}^{a}$ for a para-holomorphic function $h=h_{+} \times h_{-}: \mathbb{R}^{n} \times \mathbb{R}^{n} \supset U \rightarrow \mathbb{R} \times \mathbb{R}$. 


\subsection{5. $\operatorname{Sp}(2 n+2, \mathbb{R}) / \operatorname{Sp}(2, \mathbb{R}) \times \operatorname{Sp}(2 n, \mathbb{R})$.}

Recall that $\operatorname{Sp}(2 n, \mathbb{R}) \cong \operatorname{Sp}^{\pi}(n)$, therefore $\operatorname{Sp}(2 n+2, \mathbb{R}) / \operatorname{Sp}(2, \mathbb{R}) \times$ $\operatorname{Sp}(2 n, \mathbb{R})$ can be identified with the para-quaternionic projective space $\mathbf{H P}^{n}=\left\{\left(h_{0}, \ldots, h_{n}\right) \in \mathbf{H}^{n+1}: \sum_{a} \bar{h}_{a} h_{a} \neq 0\right\} / \mathbf{H}^{*}$, cf. Example 2.2.1.

It is useful to regard $\mathbf{H}^{n}$ as a vector space over $\mathbf{C}$ and write $\operatorname{Sp}^{\pi}(n)$ as a subgroup of $\mathrm{GL}(2 n, \mathbf{C})$ : In fact, $\mathbf{H}^{n}=\mathbf{C}^{n} \oplus i \mathbf{C}^{n}$ can be identified with the free $\mathbf{C}$ module $\mathbf{C}^{2 n}$. A $\mathbf{C}$-linear map $\mathbf{C}^{2 n} \rightarrow \mathbf{C}^{2 n}$ is $\mathbf{H}$-linear, if and only if it commutes with the right multiplication by $i$, which is a $\mathbf{C}$-antilinear involution. It is easy to see that

$$
\mathrm{GL}(n, \mathbf{H})=\left\{\left(\begin{array}{cc}
A & -\bar{B} \\
B & \bar{A}
\end{array}\right) \in \mathrm{GL}(2 n, \mathbf{C})\right\} .
$$

Let $h$ be a para-quaternionic hermitian metric on $\mathbf{H}^{n}$. The identification $\mathbf{H}=\mathbf{C} \oplus i \mathbf{C}$ yields a decomposition of $h=g \oplus i \omega$ into a para-hermitian scalar product $g$ and a $\mathbf{C}$-bilinear symplectic form $\omega$ on $\mathbf{C}^{2 n}$, which are related by $\omega=g(\cdot i, \cdot)$. Therefore an endomorphism of $\mathbf{C}^{2 n}$ preserves $h$, if and only if it preserves $g$ and $\omega$, or equivalently, if it preserves $\omega$ and is $\mathbf{H}$-linear. If $h$ is standard, so is $\omega$. This shows that

$$
\operatorname{Sp}^{\pi}(n)=\operatorname{Sp}(2 n, \mathbf{C}) \cap \operatorname{GL}(n, \mathbf{H}) .
$$

Let $G=\operatorname{Sp}^{\pi}(n+1)$.

The Lie algebra of $G$ is $\mathfrak{g}=\mathfrak{s p}^{\pi}(n+1)=\mathfrak{s p}(2 n+2, \mathbf{C}) \cap \mathfrak{g l}(n+1, \mathbf{H})$ and consists of matrices

$$
\left(\begin{array}{c|c|c}
a & -A^{*}-B^{*} & -\bar{b} \\
\hline A & \mathfrak{s p}^{\pi}(n) & -\bar{B} \\
B & \bar{A} \\
\hline b & B^{t}-A^{t} & \bar{a}
\end{array}\right) \in \mathfrak{g l}(2 n+2, \mathbf{C}), a \in j \mathbb{R} .
$$

The canonical element

$$
\xi=\left(\begin{array}{l|l|l}
j & & \\
\hline & \mathbf{0}_{2 n} & \\
\hline & & -j
\end{array}\right)
$$

leads to the twistor fibration

$$
\frac{\mathrm{Sp}^{\pi}(n+1)}{\mathrm{Sp}^{\pi}(n) \times \mathrm{U}^{\pi}(1)} \rightarrow \frac{\mathrm{Sp}^{\pi}(n+1)}{\mathrm{Sp}^{\pi}(n) \times \mathrm{Sp}^{\pi}(1)}
$$

(cf. table 1).

This is, of course, the fibration $\mathbf{C P}^{2 n+1} \rightarrow \mathbf{H P}^{n}$ of example 2.2.1. The projection map $\operatorname{Sp}^{\pi}(n+1) \rightarrow \mathrm{CP}^{2 n+1}$ sends each matrix to the $\mathbf{C}$ span of its first column. We have $G^{\mathbf{c}}=\operatorname{Sp}(2 n+2, \mathbf{C})$. The closed twistor space $\bar{Z}=G^{\mathbf{c}} / P_{-}^{\mathbf{c}}$ is the space of all (possibly degenerate) onedimensional para-complex subspaces in $\mathrm{CP}^{2 n+1}$ (by the proof proposition 2.1.2, any vector that spans such a subspace can be extended to a 
symplectic basis). The projection $G^{\mathbf{c}} \rightarrow \bar{Z}$ maps a matrix to the span of its first column.

The isomorphism of the Lie algebras

$$
\begin{aligned}
\left(\begin{array}{ccccc|c}
0 & \mathfrak{h}(n, \mathbf{C}) & \stackrel{\sim}{\rightarrow} \mathfrak{n}_{+}^{\mathbf{c}} \\
u^{1} & 0 & & & \\
\vdots & & \ddots & & \\
u^{n} & & & 0 & \\
v_{0} & v_{1} & \cdots & v_{n} & 0
\end{array}\right) & \mapsto\left(\begin{array}{cc|cc|c}
0 & & & \\
\hline \boldsymbol{u} & \mathbf{0}_{n} & & \\
\boldsymbol{v} & & \mathbf{0}_{n} & \\
\hline 2 v_{0} & \boldsymbol{v}^{t} & -\boldsymbol{u}^{t} & 0
\end{array}\right)
\end{aligned}
$$

(where $\left.\boldsymbol{u}=\left(u^{1}, \ldots, u^{n}\right)^{t}, \boldsymbol{v}=\left(v_{1}, \ldots, v_{n}\right)^{t}\right)$ yields the isomorphism

$$
\begin{aligned}
\left(\begin{array}{cccc|cc|c}
1 & & & \\
z^{1} & 1 & & & \\
\vdots & & \ddots & & \\
z^{n} & & & 1 & \\
w_{0} & w_{1} & \cdots & w_{n} & 1
\end{array}\right) & \mapsto\left(\begin{array}{ccccc}
1 & & & \\
\hline \boldsymbol{z} & \mathbf{1}_{n} & 0 & \\
\boldsymbol{w} & 0 & \mathbf{1}_{n} & \\
\hline 2 w_{0}-\boldsymbol{z}^{t} \boldsymbol{w} & \boldsymbol{w}^{t} & -\boldsymbol{z}^{t} & 1
\end{array}\right)
\end{aligned}
$$

The holomorphic horizontal maps $\mathbf{C}^{n} \supset U \rightarrow N_{+}^{\mathbf{c}}$ are given by the equations $w_{0}=h\left(z^{1}, \ldots, z^{n}\right), w_{a}=\partial h / \partial z^{a}$ for a holomorphic function $h: \mathbf{C}^{n} \supset U \rightarrow \mathbf{C}$.

The projection $N_{+} \subset \mathrm{CP}^{2 n+1} \rightarrow \mathbf{H P}^{n}$ maps this matrix to the $\mathbf{H}$-span of its first column; in conclusion, the isotropic para-pluriharmonic maps

$$
f: \mathbf{C}^{n} \supset U \rightarrow \mathbf{H P}^{n}
$$

are given by

$$
\left(z^{1}, \ldots, z^{n}\right) \mapsto \mathbf{H} \cdot\left(\begin{array}{c}
1+i\left(2 h-\sum z^{a} \partial h / \partial z^{a}\right) \\
z^{1}+i \partial h / \partial z^{1} \\
\vdots \\
z^{n}+i \partial h / \partial z^{n}
\end{array}\right)
$$




\section{CHAPTER 4}

\section{tt*-bundles and para-pluriharmonic maps}

Let $(N, J)$ be a simply connected para-complex manifold. As shown in [S1] (see also [CS] for the complex case), any para-pluriharmonic map

$$
\tilde{f}: N \rightarrow \mathrm{GL}(p+q, \mathbb{R}) / \mathrm{O}(p, q)=: \mathrm{S}_{p, q}
$$

which fulfills $R\left(d \tilde{f}\left(T^{ \pm} N\right), d \tilde{f}\left(T^{ \pm} N\right)\right)=0$ defines a metric para-tt*bundle over $N$ and vice versa. In this paragraph we relate this to the construction of the associated family (cf. section 3.1.4) and give a criterion for the map $\tilde{f}$ to be isotropic.

DEFINITION 4.0.1. Let $(N, J)$ be a simply connected para-complex manifold. A metric para-tt ${ }^{*}$-bundle $(E, D, S, g)$ over $N$ is a real vector bundle $E \rightarrow N$ endowed with a connection $D$, a (possibly indefinite) $D$-parallel fibre metric $g$ and a section $S$ of $T^{*} N \times \operatorname{End}(E)$ which is $g$-symmetric (i.e. $g\left(S_{X} \cdot, \cdot\right)=g\left(\cdot, S_{X} \cdot\right)$ ), such that the $t t^{*}$-equation

$$
R^{\theta}=0 \quad \forall \theta \in \mathbb{R}
$$

holds, where $R^{\theta}$ is the curvature tensor of the connection $\nabla_{X}^{\theta}:=D_{X}+$ $S_{e^{\theta J} X}$.

We denote by $\operatorname{Sym}_{p, q}(\mathbb{R})$ the space of symmetric $(p+q) \times(p+q)$ matrices with signature $(p, q)$. This space is identified with $\mathrm{S}_{p, q}$ via the map $\mathrm{S}_{p, q} \ni g \cdot \mathrm{O}(p, q) \mapsto\left(g^{-1}\right)^{t} \mathbf{1}_{p, q} g^{-1} \in \operatorname{Sym}_{p, q}(\mathbb{R})$, where $\mathbf{1}_{p, q}=$ $\left(\begin{array}{cc}\mathbf{1}_{p} & 0 \\ 0 & -\mathbf{1}_{q}\end{array}\right)$ is the standard diagonal matrix of signature $(p, q)$. The geodesic reflection at $e \mathrm{O}(p, q) \in \mathrm{S}_{p, q}$ yields the symmetric decomposition $\mathfrak{g l}(p+q, \mathbb{R})=\mathfrak{o}(p, q) \oplus \mathfrak{m}$, where $\mathfrak{m}=\{X \in \mathfrak{g l}(p+q, \mathbb{R})$ : $\left.X^{t} \mathbf{1}_{p, q}=\mathbf{1}_{p, q} X\right\}$. The tangent space $T_{g K} \mathrm{~S}_{p, q}$ is canonically identified with $\operatorname{Ad}_{g} \mathfrak{m}=\left\{X \in \mathfrak{g l}(p+q, \mathbb{R}): X^{t}\left(g^{-t} \mathbf{1}_{p, q} g^{-1}\right)=\left(g^{-t} \mathbf{1}_{p, q} g^{-1}\right) X\right\}$ via

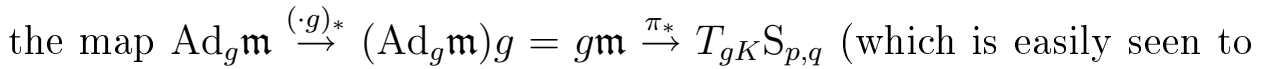
be independent of the choice of $g \in g K)$.

THEOREM. [S1, Theorem 3 and 4] Let $(N, J)$ be a simply connected para-complex manifold and let $(E, D, S, g)$ be a metric para-tt*-bundle of rank $p+q$ (and signature $(p, q)$ ) over $N$. Choose a family $s_{\theta}(x)$ : 
$\mathbb{R}^{p+q} \rightarrow E$ of $\nabla^{\theta}$-flat frames and set

$$
\begin{aligned}
f_{\theta}: N & \rightarrow \operatorname{Sym}_{p, q}(\mathbb{R}) \\
x & \mapsto s_{\theta}(x)^{*} g_{x}=\left(g\left(s_{\theta}(x)_{a}, s_{\theta}(x)_{b}\right)\right)_{a, b} \\
\tilde{f}_{\theta}: N & \stackrel{f_{\theta}}{\longrightarrow} \operatorname{Sym}_{p, q}(\mathbb{R}) \stackrel{\sim}{\rightarrow} \mathrm{S}_{p, q} \\
& \tilde{f}_{\theta}: N \frac{f_{\theta} \underset{f_{p y m}}{\longrightarrow} \operatorname{Sym}_{p, q} \stackrel{\sim}{\longrightarrow} \mathrm{S}_{p, q}}{\tilde{f}_{\theta}}
\end{aligned}
$$

Then $\tilde{f}_{\theta}$ is a 1-parameter family of para-pluriharmonic maps and

$$
d \tilde{f}_{\theta}(X)=s_{\theta}^{-1} \circ S_{e^{\theta J} X} \circ s_{\theta} .
$$

Moreover, for each $x \in N, d \tilde{f}_{\theta}$ maps $T_{x}^{+} N$ and $T_{x}^{-} N$ into abelian subalgebras of $T_{\tilde{f}_{\theta}(x)} \mathrm{S}_{p, q} \subset \mathfrak{g l}(p+q, \mathbb{R})$.

Conversely, any para-pluriharmonic map from a simply-connected paracomplex manifold $N$ into $\mathrm{S}_{p, q}$ such that the images of $T^{ \pm} N$ are abelian can be obtained in this way.

REMARK. The condition that the images of $d \tilde{f}\left(T^{ \pm} N\right)$ are abelian is equivalent to $R\left(d \tilde{f}\left(T^{ \pm} N\right), d \tilde{f}\left(T^{ \pm} N\right)\right)=0$ : Since $R$ is preserved by parallel translation, it suffices to check this assertion at $T_{o} \mathrm{~S}_{p, q} \approx \mathfrak{m}$, where $R$ is given by the Lie triple bracket, so we have to show that for $X, Y \in \mathfrak{m}:$

$$
[[X, Y], Z]=0 \forall Z \in \mathfrak{m} \quad \Leftrightarrow \quad[X, Y]=0
$$

The diagonal matrices form a maximal abelian subalgebra of $\mathfrak{g l}(p+$ $q, \mathbb{R})$, which is contained in $\mathfrak{m}$, so there is no nontrivial element of $\mathfrak{k}=\mathfrak{o}(p, q)$ that commutes with all $Z \in \mathfrak{m}$. Since $[X, Y] \in[\mathfrak{m}, \mathfrak{m}] \subset \mathfrak{k}$, the assertion follows.

This 1-parameter family $\tilde{f}_{\theta}$ of para-pluriharmonic maps is in fact an associated family in the sense of Theorem 3.1.5.

Proposition 4.0.2. The map

$$
\begin{aligned}
\Phi_{\theta}: \tilde{f}_{\theta}^{*} T \mathrm{~S}_{p, q} & \rightarrow \tilde{f}_{0}^{*} T \mathrm{~S}_{p, q} \\
X & \mapsto\left(s_{\theta}^{-1} s_{0}\right)^{-1} X\left(s_{\theta}^{-1} s_{0}\right)
\end{aligned}
$$

is a parallel bundle homomorphism preserving the curvature of $\mathrm{S}_{p, q}$, and

$$
\Phi_{\theta} \circ d \tilde{f}_{\theta}=d \tilde{f}_{0} \circ e^{\theta J} .
$$

Proof. The equation $\Phi_{\theta} \circ d \tilde{f}_{\theta}=d \tilde{f}_{0} \circ e^{\theta J}$ is immediate from $\left.(13)\right)$. $\Phi_{\theta}(x)$ is the differential of the isometry of the space $\mathrm{S}_{p, q}$, given by left multiplication by $s_{\theta}^{-1}(x) s_{0}(x) \in \mathrm{GL}(p+q, \mathbb{R})$, hence it preserves the curvature tensor. In order to verify the parallelity of $\Phi_{\theta}$, let $c:[0,1] \rightarrow$ $N$ be a curve and let $\sigma:[0,1] \rightarrow \tilde{f}_{\theta}^{*} T \mathrm{~S}_{p, q}$ be a parallel section of $\tilde{f}_{\theta}^{*} T \mathrm{~S}_{p, q}$ 
along $c$. Any such section is of the form $t \mapsto \gamma(t) \cdot X$, where $X \in \mathfrak{m}$ and $\gamma$ is the horizontal lift of $\tilde{f}_{\theta} \circ c$, i. e. $\pi \circ \gamma=\tilde{f}_{\theta} \circ c$ and $\gamma^{-1} \dot{\gamma} \in \mathfrak{m}$. We have to check that $\left(\Phi_{\theta} \circ c\right) \cdot \sigma$ is parallel, i. e. that $\tilde{\gamma}:=\left(\left(s_{0} \circ c\right)^{-1}\left(s_{\theta} \circ c\right)\right) \gamma$ is horizontal: By the definition of $s_{\theta}$ we have $D_{X} s_{\theta}=-S_{e^{\theta J}} s_{\theta}$ and $D_{X} s_{0}=-S_{X} s_{0}$, (here $D$ denotes the connection on $E \otimes \mathbb{R}^{n}$ induced by the given connection $D$ on $E$ and the trivial connection on $\mathbb{R}^{n}$ ) so $\frac{d}{d t}\left(s_{0}^{-1} s_{\theta}\right)=D_{\dot{c}}\left(s_{0}^{-1}\right) s_{\theta}+s_{0} D_{\dot{c}} s_{\theta}=s_{0}^{-1} S_{\dot{c}-e^{\theta J} \dot{c}} s_{\theta}$ and $\tilde{\gamma}^{-1} \dot{\tilde{\gamma}}=\gamma^{-1} \dot{\gamma}+$ $\gamma^{-1} \underbrace{s_{\theta}^{-1} S_{\dot{c}-e^{\theta J} \dot{c} s_{\theta}}}_{\in \operatorname{Ad}_{\gamma} \mathfrak{m}} \gamma \in \mathfrak{m}$.

PROPOSITION 4.0.3. $\tilde{f}$ is isotropic if and only if there exists a family $\alpha_{\theta}$ of D-parallel, g-orthogonal sections of $\operatorname{End}(E)$, such that

$$
S_{e^{\theta J}}=\alpha_{\theta} \circ S \circ \alpha_{\theta}^{-1} \text {. }
$$

Proof. Suppose that $\tilde{f}$ is isotropic, i. e. $\tilde{f}_{\theta}=\tilde{f}_{0}$ and therefore $d \tilde{f}_{\theta}=d \tilde{f}_{0}$ for all $\theta \in \mathbb{R}$. Define $\alpha_{\theta}:=s_{\theta} s_{0}^{-1}$. Then we see that $S_{e^{\theta J}}=\alpha_{\theta} S \alpha_{\theta}^{-1},(D \alpha) s_{0}=D\left(\alpha s_{0}\right)-\alpha D\left(s_{0}\right)=\nabla^{\theta} s_{\theta}-S_{e^{\theta J}} s_{\theta}-\alpha \nabla^{0} s_{0}+$ $\alpha S s_{0}=0$ and $g\left(s_{0}, s_{0}\right)=g\left(s_{\theta}, s_{\theta}\right)=\left(\alpha_{\theta}^{*} g\right)\left(s_{0}, s_{0}\right)$. Conversely, suppose that there exists a family $\alpha_{\theta}$ as above. Choose a $\nabla^{0}$-flat frame $s_{0}$ and set $s_{\theta}:=\alpha_{\theta} s_{0}$. Then by a similar calculation it follows that $s_{\theta}$ is $\nabla^{\theta}$-flat and that $f_{\theta}=f_{0}$.

By Corollary 3.3.7, there are no full isotropic para-pluriharmonic maps into the symmetric space $\mathrm{GL}(p+q, \mathbb{R}) / \mathrm{O}(p, q)$, since the Cartan involution $\sigma(g)=\mathbf{1}_{p, q} g^{t} \mathbf{1}_{p, q}$ is not an inner automorphism of $\mathrm{GL}(p+$ $q, \mathbb{C})$. Among the totally geodesic submanifolds is the symmetric space $\operatorname{Sp}(2 n, \mathbb{R}) / \mathrm{GL}(n, \mathbb{R}) \subset \mathrm{GL}(2 n, \mathbb{R}) / \mathrm{O}(n, n)$. Isotropic para-pluriharmonic maps into this space arise from special para-Kähler manifolds:

Example: special Kähler manifolds. Let $(N, J, g)$ be a paraKähler manifold and let $\nabla$ be a torsion-free flat connection on $T N$ such that $d^{\nabla} J=0$ and $\nabla \omega=0$, where $\omega:=g(\cdot, J \cdot)$ denotes the Kähler form of $N$. The quadruple $(N, J, g, \nabla)$ is then called an affine special paraKähler manifold. In [S1] it is shown that $(E:=T N, D:=\nabla-S, S:=$ $\left.\frac{1}{2} J(\nabla J), g\right)$ is a metric para-tt*-bundle. From the definition of $S$, one sees after short calculation that $S_{J X} Y=-J S_{X} Y=S_{X} J Y$ and

$$
S_{e^{\theta J}}=e^{-(\theta / 2) J} \circ S \circ e^{(\theta / 2) J},
$$

further $D J=0$, thus $e^{-(\theta / 2) J}$ is $D$-parallel and $g$-orthogonal, and the para-pluriharmonic map $\tilde{f}: N \rightarrow \mathrm{S}_{n, n}$ is isotropic by the above Proposition.

Indeed, $\tilde{f}$ is a para-holomorphic map into the para-hermitian symmetric space $\operatorname{Sp}(2 n, \mathbb{R}) / \mathrm{U}^{\pi}(n) \subset \mathrm{SL}(2 n, \mathbb{R}) / \mathrm{SO}(n, n)$. In [S1] this is proved by relating $\tilde{f}$ to the dual Gauß map of the canonical immersion $N \hookrightarrow \mathbf{C}^{2 n}$. A direct argument is as follows: 
The fact that the image of $\tilde{f}$ is contained in $\operatorname{Sp}(2 n, \mathbb{R}) / \mathrm{U}^{\pi}(n)=$ $\operatorname{Sp}(2 n, \mathbb{R}) /(\mathrm{O}(n, n) \cap \mathrm{Sp}(2 n, \mathbb{R}))$ is a direct consequence of $\nabla \omega=0$ and $\nabla s=0$ : The matrix-valued map $x \mapsto s^{*}(x) \omega_{x}$ is constant, and by adjusting the frame $s$ we can assume that its image is the standard skew-symmetric form $\Omega_{2 n}$. Now observe that $\gamma \in \mathrm{GL}(2 n, \mathbb{R})$ is a representative for $\tilde{f}(x)$ if and only if $s(x) \circ \gamma$ is an orthonormal basis for $g_{x}$. In particular, this is the case if $s(x) \circ \gamma$ is an orthonormal basis for the para-hermitian metric $g_{x}+j \omega_{x}$ (such bases always exist by Proposition 1.3.3. For any such $\gamma$ we have $\Omega_{2 n}=\gamma^{*} \Omega_{2 n}$, i. e. $\gamma \in \operatorname{Sp}_{2 n} \mathbb{R}$.

For the holomorphicity it is sufficient to consider one point $x_{0} \in M$. Again, by adjusting the frame $s$, we may assume $\tilde{f}\left(x_{0}\right)=e \cdot \mathrm{U}^{\pi}(n)$. If $g_{x_{0}}$ and $\omega_{x_{0}}$ have standard form in the basis $s\left(x_{0}\right)$ then so has $J_{x_{0}}$, i. e. $J_{x_{0}}=s\left(x_{0}\right) \circ J_{2 n} \circ s\left(x_{0}\right)^{-1}$. From equation (14) we get $\Phi_{\theta}=\operatorname{Ad}\left(s_{\theta}^{-1} s_{0}\right)=$ $\operatorname{Ad}\left(e^{(\theta / 2) J_{2 n}}\right)$. Differentiating the equation $\Phi_{-\theta} \circ d \tilde{f}=d \tilde{f} \circ e^{\theta J_{N}}$ yields

$$
\operatorname{ad}\left(J_{2 n} / 2\right) \circ d \tilde{f}=d \tilde{f} \circ J_{N},
$$

which shows that $\tilde{f}$ is holomorphic, because $\operatorname{ad}\left(J_{2 n} / 2\right)$ is the standard para-complex structure of $\operatorname{Sp}(2 n, \mathbb{R}) / \mathrm{U}^{\pi}(n): \operatorname{ad}\left(J_{2 n} / 2\right)$ is zero on $\mathfrak{k}=$ $\mathfrak{u}_{n}^{\pi}=\{X \in \mathfrak{s p}(2 n, \mathbb{R}): X J-J X=0\}$ and has eigenvalues \pm 1 on $\mathfrak{m}=\{X \in \mathfrak{s p}(2 n, \mathbb{R}): X J+J X=0\}$. The twistor bundle is thus trivial (cf. example 3.3.8). 


\section{Bibliography}

[AC1] D. V. Alekseevsky, V. Cortés, Classification of pseudo-Riemannian symmetric spaces of quaternionic Kähler type, Amer. Math. Soc. Transl.(2) 213 (2005), 33-62

[AC2] D. V. Alekseevsky, V. Cortés, The twistor spaces of a para-quaternionic manifold, preprint Institut Élie Cartan, Nancy, 2005/no. 55

[AM] R. Abraham, J.E. Marsden, Foundations of Mechanics, Benjamin / Cummings Publishing, Reading (Massachusetts) 1978

[Be] A. Besse, Einstein manifolds, Springer-Verlag, Berlin Heidelberg 1987

[BDM] D. E. Blair, J. Davidov, O. Muškarov, Hyperbolic Twistor Spaces, Balkan J. Geom. Appl. 5 (2000), no. 2, 9-16

[Bo] A. Borel, Linear algebraic groups, 2nd enl. ed., Springer-Verlag New York 1991

[BT] A. Borel, J. Tits, Groupes réductifs, Publications mathématiques de l'I.H.É.S., tome 27 (1965), p. 55-151

[Br] R. Bryant, Conformal and minimal immersions of compact surfaces into the 4-sphere, J. Diff. Geom. 17 (1982), 455-473

[Bu] F. E. Burstall, Minimal surfaces in quaternionic symmetric spaces, from Geometry of low-dimensional manifolds: 1, Cambrigde University Press 1990, 231-235

[BEFT] F.E. Burstall, J.H. Eschenburg, M.J. Ferreira, R. Tribuzy, Kähler submanifolds with parallel pluri-mean curvature, Differential Geometry and its Applications 20 (2004), 47-66

[BR] F. E. Burstall, J. H. Rawnsley, Twistor Theory for Riemannian Symmetric Spaces, Springer Lecture Notes in Mathematics 1424, Springer-Verlag Berlin Heidelberg 1990

[BS] F.E. Burstall, S. Salomon, Tounaments, flags and harmonic maps, Math. Ann. 277 (1987), $249-265$

[CLS] V. Cortés, M.-A. Lawn, L. Schäfer, Affine hyperspheres associated to special para-Kähler manifolds, Int. J. of Geom. Meth. in Mod. Phys. 3 (2006), nos. $\backslash 5-6,995$.

[CMMS1] V. Cortés, C. Mayer, T. Mohaupt, F. Saueressig, Special Geometry of Euclidean Supersymmetry I: Vector Multiplets, J. High Energy Phys. 03 (2004) 028, hep-th/0312001

[CMMS2] V. Cortés, C. Mayer, T. Mohaupt, F. Saueressig, Special Geometry of Euclidean Supersymmetry II: Hypermultiplets and the c-map, J. High Energy Phys. 06 (2005) 025, hep-th/0503094

[CS] V. Cortés, L. Schäfer, Topological-antitopological fusion equations, pluriharmonic maps and special Kähler manifolds in Complex, contact and symplectic manifolds, eds. O. Kowalski, E. Musso, D. Perrone, Progress in Mathematics 234, Birkhäuser 2005

[DE] J. Dorfmeister, J.-H. Eschenburg, Pluriharmonic Maps, Loop Groups and Twistor Theory, Annals of Global Analysis and Geometry 24 (2003), $301-321$ 
[DJS] A.S. Dancer, H. R. Jørgensen, A.F. Swann, Metric geometries over the split quaternions, Rend. Semin. Mat. Torino 63, No. 2 (2005), 119-139

[E] J.H. Eschenburg, Lecture Notes on Symmetric Spaces, preprint Augsburg 1997

[EQ] J. H. Eschenburg, P. Quast, Pluriharmonic Maps into Kähler Symmetric Spaces and Sym's Formula, to appear

[ET1] J.H. Eschenburg, R. Tribuzy, Existence and Uniqueness of Maps Into Affine Homogenous Spaces, Rend. Sem. Mat. Univ. Padova 89 (1993), $11-18$

[ET2] J.H. Eschenburg, R. Tribuzy, Isotropic pluriminimal submanifolds, Matématica Contemporânea 17 (1999), 171-191

[H] S. Helgason, Differential Geometry, Lie Groups and Symmetric Spaces, Academic Press 1978

[L] H. B. Lawson, Surfaces minimales et la construction de Calabi-Penrose, Sém. Bourbaki 624 (1983/84), Astérisque 121-122 (1985), 197 - 211

[MS] D. McDuff, D. Salamon, Introduction to Symplectic Topology, Oxford University Press, Oxford, New York, 1998

[O’N] B. O'Neill, Semi-Riemannian Geometry, Academic Press, New York, London, 1983

[S1] L. Schäfer, tt*-bundles in para-complex geometry, special para-Kähler manifolds and para-pluriharmonic maps, Diff. Geom. Appl. 24 (2006), 60-89

[S2] L. Schäfer, Géométrie $t t^{*}$ et applications pluriharmoniques, PhD Thesis, Nancy/Bonn 2006

[W] N.M.J. Woodhouse, Geometric Quantization, Oxford University Press, Oxford, 1994 


\section{Vita}

Name

Geburtsdatum

Geburtsort

\section{Schullaufbahn}

08/1981 - 07/1985

$08 / 1985-06 / 1994$

$06 / 1994$

\section{Zivildienst}

07/1994 - 09/1995

\section{Matthias Krahe}

29. März 1975

Koblenz

Melchiades-Best-Schule, Hillscheid

Gymnasium in Kannenbäckerland, Höhr-Grenzhausen

Abitur

\section{Studium}

10/1995 - 12/2001 Studium der Physik (4 Semester) und Mathematik an der Rheinischen Friedrich-Wilhelms-Universität, Bonn

$12 / 2001$ Diplom in Mathematik. Thema der Diplomarbeit: „Die c-Abbildung nach Ferrara und Sabharwal: Von spezieller zu quaternionischer Kählergeometrie"

01/2002 - 09/2003 Doktorand an der Universität Bonn

10/2003 - 06/2007 Doktorand an der Universität Augsburg 